\title{
INCOMPRESSIBLE FLUIDS IN THIN DOMAINS WITH NAVIER FRICTION BOUNDARY CONDITIONS (II)
}

By

\section{Luan Thach Hoang}

\section{IMA Preprint Series \#2406}

(August 2012)

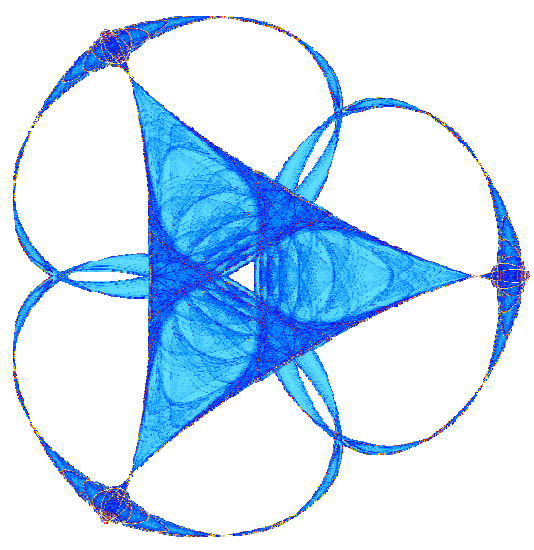

\author{
INSTITUTE FOR MATHEMATICS AND ITS APPLICATIONS \\ UNIVERSITY OF MINNESOTA \\ 400 Lind Hall \\ 207 Church Street S.E. \\ Minneapolis, Minnesota 55455-0436 \\ Phone: 612-624-6066 Fax: 612-626-7370 \\ URL: http://www.ima.umn.edu
}




\title{
INCOMPRESSIBLE FLUIDS IN THIN DOMAINS WITH NAVIER FRICTION BOUNDARY CONDITIONS (II)
}

\author{
LUAN THACH HOANG
}

\begin{abstract}
We study the Navier-Stokes equations for incompressible fluids in a three-dimensional thin two-layer domain whose top, bottom and interface boundaries are not flat. In addition to the Navier friction boundary conditions on the top and bottom boundaries of the domain, and the periodicity condition on the sides, the fluid velocities are subject to an interface boundary condition which relates the normal stress of each fluid to the relative velocity between them on the common boundary. We prove that the strong solutions exist for all time if the initial data and body force, measured in relevant norms, are appropriately large as the domain becomes very thin. In our analysis, the interface boundary condition is interpreted as a variation of the Navier boundary conditions containing an interaction part. The effect of that interaction on the Stokes operator and the nonlinear term of the Navier-Stokes equations is expressed and carefully estimated in different ways in order to obtain suitable estimates.
\end{abstract}

\section{CONTENTS}

1. Introduction 1

2. Problem formulation 3

3. Consequences of the boundary conditions 7

4. Stokes and averaging operators 16

4.1. Stokes operator and functional form of NSE 16

4.2. Averaging operators 22

5. Estimate of a nonlinear term 24

6. Global solutions 30

Appendix A. Auxiliary Inequalities 36

$\begin{array}{ll}\text { References } & 37\end{array}$

\section{INTRODUCTION}

This is a continuation of our previous work [8] in which the global existence in time of strong solutions to incompressible Navier-Stokes equations (NSE) was proved for a three-dimensional single layer thin domain under "large data conditions", that is, when the initial data and body

Date: July 9, 2012.

Key words and phrases. Navier-Stokes equations, strong solution, Navier boundary conditions, interface boundary value problem, thin domain. 
force are very large, but not arbitrarily large. The fluid is subject to the Navier friction boundary conditions on the top and bottom boundaries, and to the periodicity condition on the sides. In the current paper we study the NSE in thin two-layer domains with, in addition the mentioned Navier boundary conditions, an interface boundary condition for the stress tensors. Such an interface boundary condition is used, for example, in the coupled atmosphere-ocean models ( $[19,20]$, see also the survey [30]). The reader is referred to $[1,8-11,15,16,25]$ and references therein for NSE with Navier boundary conditions, and to [13,14, 17, 18, 21-23, 28, 29] among many others for NSE in thin domains.

Previous works on the problem are [4] and [12] which deal with domains with flat boundaries. Thanks to the simple geometry of the boundaries, (all or some) explicit solutions of the corresponding eigenvalue problems are found. Based on those, an appropriate closed subspace in which important commutativity properties of involved operators (Leray projection, or special projections, or Laplacian, or Stokes operator, or averaging operators) are available. In contrast, the domain considered in this paper has non-flat top, bottom and interface boundaries. Therefore it is impossible to find and take advantage of those explicit solutions. Also, while $[4,12]$ have free boundary conditions on the top and bottom boundaries, which correspond to the Navier conditions without friction, here we consider the Navier conditions with positive friction coefficients. We adopt the approach in $[7-9,15]$ which does not rely on any special spectral spaces. Instead, certain properties of the velocity field satisfying those boundary conditions need be realized in order to obtain suitable estimates. On one hand, the interface boundary condition is a variation of the Navier boundary conditions (see (3.5)), hence the treatment of the normal stress on the boundary resembles that in the Navier conditions. On the other hand, it can be specifically interpreted as having a Navier part and an interaction part (see (3.6) and (3.3)). The Navier part can be treated together with the conditions of the same type on the top and bottom boundaries, while the interaction part requires extra work. Thanks to such coherent treatments, we can take advantage of our previous works on the Navier conditions and prove the global existence of strong solutions for this involved case within a reasonable length.

Our result (Theorems 6.2 and 6.3) is comparable to those obtained in $[7,9,15]$ for single layer domains. It only differs from $[4,12]$ for two-layer domains only on the technical dependence on the domain's thickness $\varepsilon$. Briefly speaking, the global-in-time strong solutions exist even when the initial data has large $H^{1}$-norm and the body force has large $L^{2}$-norm, both expressed in terms of $\varepsilon$. Moreover, thanks to strictly positive friction coefficients, none of the zero average condition in $[4,12]$, or the orthogonality condition in [15], or the generic domain condition in $[8,9]$, is imposed. It is noteworthy that our result shows that the larger friction we have on the top and bottom boundaries, the larger (in $L^{2}$-norm) initial and body force are allowed. The reader is referred to Remark 6.5 for more discussions on the result.

The article is organized as follows. In section 2, we introduce the problem, formulation, notation and basic assumptions. In section 3, properties of vector fields satisfying the boundary conditions are presented. These include both their behavior on the boundaries and their norm 
estimates on the whole domain. In section 4, we define the Stokes operator $A$ corresponding to our boundary conditions, and derive the functional form of the NSE. Relations between Lebesgue and Sobolev norms with $A$-related norms are obtained. Particular is the $H^{2}$-norm relation and comparison estimate in Propositions 4.3, 4.4 and Corollary 4.5. We also define the averaging operators $M_{0}$ and $M$ in subsection 4.2 which play crucial roles in our result's formulation and in obtaining various estimates. Inequalities of Sobolev, Poincaré and Agmon types for these averages and their corresponding oscillations are derived with constants depending explicitly on the domain's thickness and fluids' viscosities. In section 5, the key nonlinear estimate is proved under the main assumption (5.1) on the viscosities and friction coefficients. It is derived with the desired boundedness of the term $\varepsilon\left\|A^{1 / 2} \boldsymbol{u}\right\|_{L^{2}}^{2}$ kept in mind. In section 6, we state and prove the main theorem of the paper (Theorem 6.2). Its argument is arranged for clarity with the use of Lemma 6.4. This theorem is restated in the form of Theorem 6.3 without using the Stokes operator. Final comments are given in Remark 6.5. The Appendix recalls some auxiliary inequalities for thin domains.

\section{PROBLEM FORMULATION}

Let $\mathbb{T}^{2}$ be the two-dimensional torus $(\mathbb{R} / \mathbb{Z})^{2}$ and $\varepsilon \in(0,1]$. Let $\Omega_{+}^{\varepsilon}$ and $\Omega_{-}^{\varepsilon}$ be two layers

$$
\begin{aligned}
& \Omega_{+}^{\varepsilon}=\left\{\left(x^{\prime}, x_{3}\right): x^{\prime} \in \mathbb{T}^{2}, h_{0}^{\varepsilon}\left(x^{\prime}\right)<x_{3}<h_{+}^{\varepsilon}\left(x^{\prime}\right)\right\}, \\
& \Omega_{-}^{\varepsilon}=\left\{\left(x^{\prime}, x_{3}\right): x^{\prime} \in \mathbb{T}^{2}, h_{-}^{\varepsilon}\left(x^{\prime}\right)<x_{3}<h_{0}^{\varepsilon}\left(x^{\prime}\right)\right\},
\end{aligned}
$$

where $h_{0}^{\varepsilon}=\varepsilon g_{0}, h_{+}^{\varepsilon}=\varepsilon\left(g_{0}+g_{+}\right)$and $h_{-}^{\varepsilon}=\varepsilon\left(g_{0}-g_{-}\right)$, with the functions $g_{0}\left(x^{\prime}\right), g_{+}\left(x^{\prime}\right)$ and $g_{-}\left(x^{\prime}\right)$ belonging to $C^{3}\left(\mathbb{T}^{2}\right)$, and $g_{+}, g_{-}$positive everywhere. Hence there is a positive constant $c_{0}>0$ such that

$$
c_{0}^{-1} \leq g_{0}, g_{+}, g_{-} \leq c_{0} \quad \text { in } \mathbb{T}^{2}, \quad\left\|g_{0}\right\|_{C^{3}\left(\mathbb{T}^{2}\right)},\left\|g_{+}\right\|_{C^{3}\left(\mathbb{T}^{2}\right)},\left\|g_{-}\right\|_{C^{3}\left(\mathbb{T}^{2}\right)} \leq c_{0} .
$$

We will use the same notation $c_{0}$ to denote positive constants in our assumptions below.

We define our two-layer domain by $\Omega^{\varepsilon}=\Omega_{+}^{\varepsilon} \cup \Omega_{-}^{\varepsilon}$. The top and bottom boundaries of $\Omega^{\varepsilon}$ are

$$
\begin{aligned}
& \Gamma_{+}^{\varepsilon}=\left\{\left(x^{\prime}, x_{3}\right): x^{\prime} \in \mathbb{T}^{2}, x_{3}=h_{+}^{\varepsilon}\left(x^{\prime}\right)\right\}, \\
& \Gamma_{-}^{\varepsilon}=\left\{\left(x^{\prime}, x_{3}\right): x^{\prime} \in \mathbb{T}^{2}, x_{3}=h_{-}^{\varepsilon}\left(x^{\prime}\right)\right\},
\end{aligned}
$$

while the interface boundary is

$$
\Gamma_{0}^{\varepsilon}=\left\{\left(x^{\prime}, x_{3}\right): x^{\prime} \in \mathbb{T}^{2}, x_{3}=h_{0}^{\varepsilon}\left(x^{\prime}\right)\right\} .
$$

Each layer $\Omega_{+}^{\varepsilon}$, resp. $\Omega_{-}^{\varepsilon}$, is occupied with an incompressible viscous fluid of velocity $u_{+}=$ $u_{+}^{\varepsilon}(x, t)$ for $x \in \Omega_{+}^{\varepsilon}$ and $t \in \mathbb{R}$, resp. $u_{-}=u_{-}^{\varepsilon}(x, t)$ for $x \in \Omega_{+}^{\varepsilon}$ and $t \in \mathbb{R}$, pressure $p_{+}=p_{+}^{\varepsilon}(x, t)$, resp. $p_{-}=p_{-}^{\varepsilon}(x, t)$, having kinetic viscosity $\nu_{+}=\nu_{+}^{\varepsilon}$, resp. $\nu_{-}=\nu_{-}^{\varepsilon}$. Each $u_{+}(x, t)$, resp. $u_{-}(x, t)$ satisfies the corresponding NSE on $\Omega_{+}^{\varepsilon}$, resp. $\Omega_{-}^{\varepsilon}$ with an outer body force $f_{+}=f_{+}^{\varepsilon}(x, t)$, resp. $f_{-}=f_{-}^{\varepsilon}(x, t)$. 
For each vector field $u$, we denote by $\nabla u$ the $3 \times 3$ matrix of its partial derivatives, that is, $(\nabla u)_{i j}=\frac{\partial u_{i}}{\partial x_{j}}$, for $i, j=1,23$. We also denote

$$
D u=\frac{\nabla u+(\nabla u)^{*}}{2} \text { where }(\nabla u)^{*} \text { is the transpose matrix of } \nabla u .
$$

Below, we will drop the superscript $\varepsilon$ except for the domain notation $\Omega^{\varepsilon}, \Omega_{+}^{\varepsilon}, \Omega_{-}^{\varepsilon}$.

The velocity fields $u_{+}$and $u_{-}$satisfy the following boundary conditions.

- The Navier friction conditions on the top and bottom boundaries:

$$
\begin{aligned}
& u_{+} \cdot N_{+}=0, \quad\left[\nu_{+}\left(D u_{+}\right) N_{+}\right]_{\tan }+\gamma_{+} u_{+}=0 \text { on } \Gamma_{+}, \\
& u_{-} \cdot N_{-}=0, \quad\left[\nu_{-}\left(D u_{-}\right) N_{-}\right]_{\tan }+\gamma_{-} u_{-}=0 \text { on } \Gamma_{-},
\end{aligned}
$$

where $\gamma_{+}=\gamma_{+}^{\varepsilon}$ and $\gamma_{-}=\gamma_{-}^{\varepsilon}$ are positive constants. Above, $N_{+}$, resp. $N_{-}$, is the outward normal vector to the boundary of $\Omega_{+}^{\varepsilon}$, resp. $\Omega_{-}^{\varepsilon}$. The subscript "tan" denotes the tangential component of a vector on the boundary.

- The interface boundary condition on $\Gamma_{0}$ :

$$
\begin{aligned}
& u_{+} \cdot N_{+}=0, \quad\left[\nu_{+}\left(D u_{+}\right) N_{+}\right]_{\tan }+\gamma_{0}\left(u_{+}-u_{-}\right)=0, \\
& u_{-} \cdot N_{-}=0, \quad\left[\nu_{-}\left(D u_{-}\right) N_{-}\right]_{\tan }+\gamma_{0}\left(u_{-}-u_{+}\right)=0,
\end{aligned}
$$

where $\gamma_{0}=\gamma_{0}^{\varepsilon}$ is a positive constant.

For a unified treatment of equations on both $\Omega_{+}^{\varepsilon}$ and $\Omega_{-}^{\varepsilon}$, we introduce the index notation $\iota \in\{+,-\}$ and set

$$
-\iota= \begin{cases}- & \text { if } \iota=+ \\ + & \text { if } \iota=-\end{cases}
$$

In calculations, when not used as an index, i.e., not a subscript, $\iota=+,-$, is interpreted as $(+1),(-1)$, respectively. Therefore the previous convention (2.5) for $-\iota$ agrees with these numeric values.

With the notation $\iota$, we rewrite $h_{\iota}=\varepsilon\left(g_{0}+\iota g_{\iota}\right)$ and have

$$
\begin{aligned}
& \Omega_{\iota}^{\varepsilon}=\left\{\left(x^{\prime}, x_{3}\right): x^{\prime} \in \mathbb{T}^{2}, x_{3} \text { is between } h_{0}\left(x^{\prime}\right) \text { and } h_{\iota}\left(x^{\prime}\right)\right\}, \\
& \Gamma_{\iota}=\left\{\left(x^{\prime}, x_{3}\right): x^{\prime} \in \mathbb{T}^{2}, x_{3}=h_{\iota}\left(x^{\prime}\right)\right\} .
\end{aligned}
$$

Note that $\partial \Omega_{\iota}^{\varepsilon}=\Gamma_{\iota} \cup \Gamma_{0}$ and $N_{-\iota}=-N_{\iota}$ on $\Gamma_{0}$.

We also rewrite the Navier boundary conditions (2.1) and (2.2) as

$$
u_{\iota} \cdot N_{\iota}=0, \quad\left[\nu_{\iota} D\left(u_{\iota}\right) N_{\iota}\right]_{\tan }+\gamma_{\iota} u_{\iota}=0 \text { on } \Gamma_{\iota} \text { for } \iota=+,-,
$$

and rewrite the boundary conditions (2.3) and (2.4) as

$$
u_{\iota} \cdot N_{\iota}=0, \quad\left[\nu_{\iota} D\left(u_{\iota}\right) N_{\iota}\right]_{\tan }+\gamma_{0}\left(u_{\iota}-u_{-\iota}\right)=0 \text { on } \Gamma_{0} \text { for } \iota=+,-.
$$


To avoid writing the + and - components repeatedly like in (2.6) and (2.7), we pair them together into vectors:

$$
\begin{aligned}
& \boldsymbol{\Omega}^{\varepsilon}=\left[\Omega_{+}^{\varepsilon}, \Omega_{-}^{\varepsilon}\right], \partial \boldsymbol{\Omega}^{\varepsilon}=\left[\partial \Omega_{+}^{\varepsilon}, \partial \Omega_{-}^{\varepsilon}\right], \boldsymbol{\Gamma}=\left[\Gamma_{+}, \Gamma_{-}\right] \text {and } \boldsymbol{\Gamma}_{0}=\left[\Gamma_{0}, \Gamma_{0}\right], \\
& \boldsymbol{u}=\left[\boldsymbol{u}_{+}, \boldsymbol{u}_{-}\right], \boldsymbol{p}=\left[p_{+}, p_{-}\right], \boldsymbol{f}=\left[f_{+}, f_{-}\right] \text {and } \boldsymbol{\nu}=\left[\nu_{+}, \nu_{-}\right] .
\end{aligned}
$$

The initial value problem for the NSE in $\Omega^{\varepsilon}$ is written in the form:

$$
\begin{cases}\frac{\partial \boldsymbol{u}}{\partial t}+(\boldsymbol{u} \cdot \nabla) \boldsymbol{u}-\nu \Delta \boldsymbol{u}=-\nabla \boldsymbol{p}+\boldsymbol{f} & \text { on } \boldsymbol{\Omega}^{\varepsilon} \times(0, \infty), \\ \operatorname{div} \boldsymbol{u}=0 & \text { on } \boldsymbol{\Omega}^{\varepsilon} \times(0, \infty), \\ \boldsymbol{u}(\boldsymbol{x}, 0)=\boldsymbol{u}^{0}(\boldsymbol{x}) & \text { on } \boldsymbol{\Omega}^{\varepsilon},\end{cases}
$$

where $\boldsymbol{u}^{0}=\left[u_{+}^{0}, u_{-}^{0}\right]$ is the known initial velocity field.

We will write $u_{+}=\left(u_{+, 1}, u_{+, 2}, u_{+, 3}\right), u_{-}=\left(u_{-, 1}, u_{-, 2}, u_{-, 3}\right)$ and $\boldsymbol{u}=\left(\boldsymbol{u}_{1}, \boldsymbol{u}_{2}, \boldsymbol{u}_{3}\right)$ where $\boldsymbol{u}_{i}=\left[u_{+, i}, u_{-, i}\right]$ for each $i=1,2,3$.

Denoting $\boldsymbol{N}=\left[N_{+}, N_{-}\right]$and $\gamma=\left[\gamma_{+}, \gamma_{-}\right]$, we rewrite the set of boundary conditions (2.6) and (2.7) in the following compact forms:

$$
\begin{gathered}
\boldsymbol{u} \cdot \boldsymbol{N}=0 \quad \text { on } \boldsymbol{\Gamma} \\
{[\boldsymbol{\nu}(D \boldsymbol{u}) \boldsymbol{N}]_{\tan }+\boldsymbol{\gamma} \boldsymbol{u}=0 \quad \text { on } \boldsymbol{\Gamma},}
\end{gathered}
$$

and

$$
\begin{gathered}
\boldsymbol{u} \cdot \boldsymbol{N}=0 \quad \text { on } \boldsymbol{\Gamma}_{0} \\
{[\boldsymbol{\nu}(D \boldsymbol{u}) \boldsymbol{N}]_{\tan }+\gamma_{0}(\boldsymbol{u}-S \boldsymbol{u})=0 \quad \text { on } \boldsymbol{\Gamma}_{0}}
\end{gathered}
$$

where

$$
S \boldsymbol{u}=S\left[u_{+}, u_{-}\right] \stackrel{\text { def }}{=}\left[u_{-}, u_{+}\right] .
$$

The slip conditions (2.10) and (2.12) can be combined into

$$
\boldsymbol{u} \cdot \boldsymbol{N}=0 \quad \text { on } \partial \boldsymbol{\Omega}^{\varepsilon} .
$$

We now discuss the relations between viscosities $\nu_{+}^{\varepsilon}, \nu_{-}^{\varepsilon}$ and friction coefficients $\gamma_{+}^{\varepsilon}, \gamma_{-}^{\varepsilon}, \gamma_{0}^{\varepsilon}$ as $\varepsilon \rightarrow 0$. (We remind the reader that the superscript $\varepsilon$ here is an index, not an exponent.)

First we assume that $\nu_{+}$and $\nu_{-}$are comparable to each other as $\varepsilon \rightarrow 0$, that is,

$$
0<\liminf _{\varepsilon \searrow 0} \frac{\nu_{\iota}^{\varepsilon}}{\sqrt{\left|\nu_{+}^{\varepsilon}\right|^{2}+\left|\nu_{-}^{\varepsilon}\right|^{2}}} \leq \limsup _{\varepsilon \searrow 0} \frac{\nu_{\iota}^{\varepsilon}}{\sqrt{\left|\nu_{+}^{\varepsilon}\right|^{2}+\left|\nu_{-}^{\varepsilon}\right|^{2}}}<\infty, \quad \iota=+,-.
$$

Assume that there exist $\delta_{+}, \delta_{-}, \delta_{0} \in[0,1]$ such that

$$
0<\liminf _{\varepsilon \searrow 0} \varepsilon^{-\delta_{\iota}} \frac{\gamma_{\iota}^{\varepsilon}}{\nu_{\iota}^{\varepsilon}} \leq \limsup _{\varepsilon \searrow 0} \varepsilon^{-\delta_{\iota}} \frac{\gamma_{\iota}^{\varepsilon}}{\nu_{\iota}^{\varepsilon}}<\infty \quad \text { for } \iota=+,-
$$

and

$$
0<\liminf _{\varepsilon \searrow 0} \varepsilon^{-\delta_{0}} \frac{\gamma_{0}^{\varepsilon}}{\nu_{\iota}^{\varepsilon}} \leq \limsup _{\varepsilon \searrow 0} \varepsilon^{-\delta_{0}} \frac{\gamma_{0}^{\varepsilon}}{\nu_{\iota}^{\varepsilon}}<\infty
$$


For the sake of simplicity, we consider through out that

$$
0<\delta_{+}=\delta_{-}=\delta \leq \delta_{0} \leq 1
$$

Taking into account the above conditions, without loss of generality, we assume

$$
\begin{gathered}
\nu_{+}, \nu_{-} \geq c_{0}^{-1}|\boldsymbol{\nu}|_{\star}, \\
c_{0}^{-1} \varepsilon^{\delta}|\boldsymbol{\nu}|_{\star} \leq \gamma_{-}, \gamma_{+} \leq c_{0} \varepsilon^{\delta}|\boldsymbol{\nu}|_{\star} \quad \text { and } \quad c_{0}^{-1} \varepsilon^{\delta_{0}}|\boldsymbol{\nu}|_{\star} \leq \gamma_{0} \leq c_{0} \varepsilon^{\delta_{0}}|\boldsymbol{\nu}|_{\star},
\end{gathered}
$$

for all $\varepsilon \in(0,1]$, where $|\boldsymbol{\nu}|_{\star}=\left(\nu_{+}^{2}+\nu_{-}^{2}\right)^{1 / 2}$.

Notation. Concerning the vectors in (2.8), for any $f, \boldsymbol{f}=\left[f_{+}, f_{-}\right]$and $\boldsymbol{u}=\left[u_{+}, u_{-}\right]$we denote

$$
f(\boldsymbol{u})=\left[f\left(u_{+}\right), f\left(u_{-}\right)\right] \quad \text { and } \quad \boldsymbol{f}(\boldsymbol{u})=\left[f_{+}\left(u_{+}\right), f_{-}\left(u_{-}\right)\right] .
$$

For example, $|\boldsymbol{u}|=\left[\left|u_{+}\right|,\left|u_{-}\right|\right]$and $\boldsymbol{\nu} \Delta \boldsymbol{u} \cdot \boldsymbol{v}=\left[\nu_{+} \Delta u_{+} \cdot v_{+}, \nu_{-} \Delta u_{-} \cdot v_{-}\right]$.

The usual Euclidean length of vector $\boldsymbol{u}$ is denoted by

$$
|\boldsymbol{u}|_{\star}=\left(\left|u_{+}\right|^{2}+\left|u_{-}\right|^{2}\right)^{1 / 2} .
$$

Obviously,

$$
|S \boldsymbol{u}|_{\star}=|\boldsymbol{u}|_{\star}
$$

For integrals, we use the notation

$$
\int_{\Omega^{\varepsilon}} \boldsymbol{f}(\boldsymbol{u}) d \boldsymbol{x}=\left[\int_{\Omega_{+}^{\varepsilon}} f_{+}\left(u_{+}\right) d x, \int_{\Omega_{-}^{\varepsilon}} f_{-}\left(u_{-}\right) d x\right],
$$

while

$$
\int_{\boldsymbol{\Omega}^{\varepsilon}}^{\star} \boldsymbol{f}(\boldsymbol{u}) d \boldsymbol{x}=\int_{\Omega_{+}^{\varepsilon}} f_{+}\left(u_{+}\right) d x+\int_{\Omega_{-}^{\varepsilon}} f_{-}\left(u_{-}\right) d x .
$$

Similar notation is used for surface integrals on $\partial \boldsymbol{\Omega}^{\varepsilon}, \Gamma$ and $\boldsymbol{\Gamma}_{0}$.

The Lebesgue and Sobolev spaces on $\Omega^{\varepsilon}$ are naturally defined as follows. Let

$$
L^{2}\left(\boldsymbol{\Omega}^{\varepsilon}\right)=L^{2}\left(\Omega_{+}^{\varepsilon}\right) \oplus L^{2}\left(\Omega_{-}^{\varepsilon}\right)=\left\{\boldsymbol{u}=\left[u_{+}, u_{-}\right]: u_{\iota} \in L^{2}\left(\Omega_{\iota}^{\varepsilon}\right), \iota=+,-\right\} .
$$

This space is equipped with the inner product

$$
\langle\boldsymbol{u}, \boldsymbol{v}\rangle=\left\langle u_{+}, v_{+}\right\rangle_{L^{2}\left(\Omega_{+}^{\varepsilon}\right)}+\left\langle u_{-}, v_{-}\right\rangle_{L^{2}\left(\Omega_{-}^{\varepsilon}\right)}=\int_{\boldsymbol{\Omega}^{\varepsilon}}^{\star} \boldsymbol{u} \cdot \boldsymbol{v} d \boldsymbol{x} .
$$

We denote the corresponding norm by $\|\boldsymbol{u}\|_{L^{2}}$, while denoting

$$
[[\boldsymbol{u}]]_{L^{2}}=\left[\left\|u_{+}\right\|_{L^{2}\left(\Omega_{+}^{\varepsilon}\right)},\left\|u_{-}\right\|_{L^{2}\left(\Omega_{-}^{\varepsilon}\right)}\right] \neq\|\boldsymbol{u}\|_{L^{2}}=\left(\left\|u_{+}\right\|_{L^{2}\left(\Omega_{+}^{\varepsilon}\right)}^{2}+\left\|u_{+}\right\|_{L^{2}\left(\Omega_{-}^{\varepsilon}\right)}^{2}\right)^{1 / 2} .
$$

The spaces $H^{1}\left(\Omega^{\varepsilon}\right)$ and $H^{2}\left(\Omega^{\varepsilon}\right)$ with their norms are defined similarly. Same definitions and notations apply to the spaces $\left(L^{2}\left(\boldsymbol{\Omega}^{\varepsilon}\right)\right)^{k},\left(H^{1}\left(\boldsymbol{\Omega}^{\varepsilon}\right)\right)^{k}$ and $\left(H^{2}\left(\boldsymbol{\Omega}^{\varepsilon}\right)\right)^{k}$, for $k=1,2,3, \ldots$ The norms in those spaces will be denoted by the same notations $\|\cdot\|_{L^{2}},\|\cdot\|_{H^{1}}$ and $\|\cdot\|_{H^{2}}$, disregarding $k$. Such disregard of $k$ will be used also for pairs of norms $[[\boldsymbol{u}]]_{L^{2}},\left[[\boldsymbol{u}]_{H^{1}}\right.$ and $[[\boldsymbol{u}]]_{H^{2}}$. 
Throughout, the expression

$$
\left[u_{+}, u_{-}\right]=(\leq, \geq)\left[v_{+}, v_{-}\right]+C \text { means } u_{+}=(\leq, \geq) v_{+}+C \text { and } u_{-}=(\leq, \geq) v_{-}+C,
$$

where $C$ is a number. Also, we write

$$
f_{1}=(\leq) f_{2}+O\left(\varepsilon^{\alpha}\right) f_{3} \text { where } f_{3} \geq 0, \text { to indicate that } f_{1}-f_{2}=(\leq) f_{4} \text { with }\left|f_{4}\right| \leq C \varepsilon^{\alpha} f_{3},
$$

when $\varepsilon>0$ is sufficiently small.

\section{CONSEQUENCES OF THE BOUNDARY CONDITIONS}

We present in this section some properties of the vector fields satisfying the Navier and/or interface boundary conditions. They extend the similar ones for Navier friction boundary conditions in [2,7-9]. For a function $u(x)$ and a vector $\tau \in \mathbb{R}^{3}$ not necessarily having unit length we denote

$$
\frac{\partial u(x)}{\partial \tau}=\lim _{h \rightarrow 0} \frac{u(x+h \tau)-u(x)}{h} .
$$

We restate Lemma 4.1 of [8] for our two-layer domain (see also [2]):

Lemma 3.1 ( [8]). Let $\boldsymbol{u}$ satisfy (2.10) and $\boldsymbol{\tau}$ be a tangential vector field to $\boldsymbol{\Gamma}$. Then

$$
\frac{\partial \boldsymbol{u}}{\partial \boldsymbol{\tau}} \cdot \boldsymbol{N}+\frac{\partial \boldsymbol{N}}{\partial \boldsymbol{\tau}} \cdot \boldsymbol{u}=0 \text { on } \boldsymbol{\Gamma} .
$$

If, in addition, $\boldsymbol{u}$ satisfies (2.11) then

$$
\frac{\partial \boldsymbol{u}}{\partial \boldsymbol{N}} \cdot \boldsymbol{\tau}=\boldsymbol{u} \cdot\left\{\frac{\partial \boldsymbol{N}}{\partial \boldsymbol{\tau}}-2 \frac{\gamma}{\boldsymbol{\nu}} \boldsymbol{\tau}\right\} \quad \text { on } \quad \boldsymbol{\Gamma}
$$

and

$$
\boldsymbol{N} \times(\nabla \times \boldsymbol{u})=2 \boldsymbol{N} \times\left\{\boldsymbol{n} \times\left((\nabla \boldsymbol{n})^{*} u\right)-\frac{\boldsymbol{\gamma}}{\boldsymbol{\nu}} \boldsymbol{N} \times \boldsymbol{u}\right\} \quad \text { on } \quad \boldsymbol{\Gamma},
$$

where $\boldsymbol{n}$ is any $C^{1}$-extension of one of the four vector fields $\left[ \pm N_{+}, \pm N_{-}\right]$from $\boldsymbol{\Gamma}$ to its neighborhood.

For the interface boundary, we obtain similar properties:

Lemma 3.2. Let $\boldsymbol{u}$ satisfy (2.12) and $\boldsymbol{\tau}$ be a tangential vector field to $\boldsymbol{\Gamma}_{0}$. Then

$$
\frac{\partial \boldsymbol{u}}{\partial \boldsymbol{\tau}} \cdot \boldsymbol{N}+\frac{\partial \boldsymbol{N}}{\partial \boldsymbol{\tau}} \cdot \boldsymbol{u}=0 \text { on } \boldsymbol{\Gamma}_{0}
$$

If, in addition, $\boldsymbol{u}$ satisfies (2.13), then

$$
\frac{\partial \boldsymbol{u}}{\partial \boldsymbol{N}} \cdot \boldsymbol{\tau}=\boldsymbol{u} \cdot \frac{\partial \boldsymbol{N}}{\partial \boldsymbol{\tau}}-2 \frac{\gamma_{0}}{\boldsymbol{\nu}}(\boldsymbol{u}-S \boldsymbol{u}) \cdot \boldsymbol{\tau} \quad \text { on } \quad \boldsymbol{\Gamma}_{0}
$$

and

$$
\boldsymbol{N} \times(\nabla \times \boldsymbol{u})=2 \boldsymbol{N} \times\left\{\boldsymbol{n} \times\left((\nabla \boldsymbol{n})^{*} \boldsymbol{u}\right)-\frac{\gamma_{0}}{\boldsymbol{\nu}} \boldsymbol{N} \times \boldsymbol{u}\right\}-2 \frac{\gamma_{0}}{\boldsymbol{\nu}} S \boldsymbol{u} \quad \text { on } \quad \boldsymbol{\Gamma}_{0},
$$

where $\boldsymbol{n}$ is any $C^{1}$-extension of one of the four vector fields $\left[ \pm N_{+}, \pm N_{-}\right]$from $\boldsymbol{\Gamma}_{0}$ to its neighborhood. 
Proof. Identity (3.4) is the same as (3.1). For (3.5), we observe that on $\Gamma_{0}$, the relation (2.13) implies $\boldsymbol{\nu}[(D \boldsymbol{u}) \boldsymbol{N}] \cdot \boldsymbol{\tau}=-\gamma_{0}(\boldsymbol{u}-S \boldsymbol{u}) \cdot \boldsymbol{\tau}$, which in turn yields

$$
(\nabla \boldsymbol{u}) \boldsymbol{N} \cdot \boldsymbol{\tau}+(\nabla \boldsymbol{u}) \boldsymbol{\tau} \cdot \boldsymbol{N}=-2 \frac{\gamma_{0}}{\boldsymbol{\nu}}(\boldsymbol{u}-S \boldsymbol{u}) \cdot \boldsymbol{\tau}
$$

hence

$$
\frac{\partial \boldsymbol{u}}{\partial \boldsymbol{N}} \cdot \boldsymbol{\tau}+\frac{\partial \boldsymbol{u}}{\partial \boldsymbol{\tau}} \cdot \boldsymbol{N}=-2 \frac{\gamma_{0}}{\boldsymbol{\nu}}(\boldsymbol{u}-S \boldsymbol{u}) \cdot \boldsymbol{\tau}
$$

Therefore (3.5) follows from (3.7) and (3.4).

We now prove (3.6). Let $\boldsymbol{\omega}=\nabla \times \boldsymbol{u}$. Let $\boldsymbol{\tau}_{1}$ and $\boldsymbol{\tau}_{2}$ be two unit tangential vectors on $\boldsymbol{\Gamma}_{0}$ which are orthogonal to each other. Then

$$
0=\frac{\partial(\boldsymbol{u} \cdot \boldsymbol{n})}{\partial \boldsymbol{\tau}_{1}} \boldsymbol{\tau}_{2}-\frac{\partial(\boldsymbol{u} \cdot \boldsymbol{n})}{\partial \boldsymbol{\tau}_{2}} \boldsymbol{\tau}_{1}=\left(\boldsymbol{\tau}_{1} \times \boldsymbol{\tau}_{2}\right) \times \nabla(\boldsymbol{u} \cdot \boldsymbol{n}) .
$$

Since $\boldsymbol{n}= \pm \boldsymbol{\tau}_{1} \times \boldsymbol{\tau}_{2}$, we have on $\boldsymbol{\Gamma}_{0}$ that $\boldsymbol{n} \times \nabla(\boldsymbol{u} \cdot \boldsymbol{n})=0$, hence

$$
\begin{aligned}
0 & =\boldsymbol{n} \times\left[(\nabla \boldsymbol{u})^{*} \boldsymbol{n}\right]+\boldsymbol{n} \times\left[(\nabla \boldsymbol{n})^{*} u\right] \\
& =\boldsymbol{n} \times[(D \boldsymbol{u}) \boldsymbol{n}-(K \boldsymbol{u}) \boldsymbol{n}]+\boldsymbol{n} \times\left[(\nabla \boldsymbol{n})^{*} \boldsymbol{u}\right],
\end{aligned}
$$

where $K \boldsymbol{u}=\frac{1}{2}\left\{\nabla \boldsymbol{u}-(\nabla \boldsymbol{u})^{*}\right\}$. Note that

$$
\boldsymbol{n} \times[(D \boldsymbol{u}) \boldsymbol{n}-(K \boldsymbol{u}) \boldsymbol{n}]=\boldsymbol{N} \times[(D \boldsymbol{u}) \boldsymbol{N}-(K \boldsymbol{u}) \boldsymbol{N}],
$$

with

and, thanks to (2.13),

$$
(K \boldsymbol{u}) \boldsymbol{N}=\frac{1}{2} \boldsymbol{\omega} \times \boldsymbol{N}
$$

$$
\boldsymbol{N} \times[(D \boldsymbol{u}) \boldsymbol{N}]=\boldsymbol{N} \times[(D \boldsymbol{u}) \boldsymbol{N}]_{\tan }=-\frac{\gamma_{0}}{\boldsymbol{\nu}} \boldsymbol{N} \times(\boldsymbol{u}-S \boldsymbol{u}) .
$$

Thus we have from (3.8) that

$$
0=-\frac{\gamma_{0}}{\boldsymbol{\nu}} \boldsymbol{N} \times(\boldsymbol{u}-S \boldsymbol{u})-\frac{1}{2} \boldsymbol{N} \times(\boldsymbol{\omega} \times \boldsymbol{N})+\boldsymbol{n} \times\left[(\nabla \boldsymbol{n})^{*} \boldsymbol{u}\right] .
$$

Therefore

$$
-\boldsymbol{N} \times(\boldsymbol{N} \times \boldsymbol{\omega})=2 \boldsymbol{n} \times\left[(\nabla \boldsymbol{n})^{*} \boldsymbol{u}\right]-2 \frac{\gamma_{0}}{\boldsymbol{\nu}} \boldsymbol{N} \times(\boldsymbol{u}-S \boldsymbol{u}) .
$$

Applying $(\boldsymbol{N} \times)$ to (3.9) and using the identity $a \times(a \times(a \times b)))=-|a|^{2}(a \times b)$, we obtain

$$
\boldsymbol{N} \times \boldsymbol{\omega}=2 \boldsymbol{N} \times\left\{\boldsymbol{n} \times\left((\nabla \boldsymbol{n})^{*} \boldsymbol{u}\right)-\frac{\gamma_{0}}{\boldsymbol{\nu}} \boldsymbol{N} \times(\boldsymbol{u}-S \boldsymbol{u})\right\} \quad \text { on } \quad \boldsymbol{\Gamma}_{0} .
$$

Note that $\boldsymbol{N} \cdot S \boldsymbol{u}=-S \boldsymbol{N} \cdot S \boldsymbol{u}=0$ on $\boldsymbol{\Gamma}_{0}$. Then $\boldsymbol{N} \times(\boldsymbol{N} \times S \boldsymbol{u})=-S \boldsymbol{u}$ on $\boldsymbol{\Gamma}_{0}$. Therefore (3.6) follows (3.10).

To make use of properties (3.3) and (3.6), we specify below extensions of $N_{\iota}$ from $\Gamma_{\iota}$ to the whole domain $\mathbb{T}^{2} \times \mathbb{R}$. We define for $x=\left(x^{\prime}, x_{3}\right)=\left(x_{1}, x_{2}, x_{3}\right) \in \mathbb{T}^{2} \times \mathbb{R}$ that

$$
\mathcal{N}_{\iota}\left(x^{\prime}, x_{3}\right)=\frac{\iota\left(-\partial_{1} h_{\iota}\left(x^{\prime}\right),-\partial_{2} h_{\iota}\left(x^{\prime}\right), 1\right)}{\sqrt{1+\left|\nabla_{2} h_{\iota}\left(x^{\prime}\right)\right|^{2}}},
$$


where $\nabla_{2}=\left(\partial / \partial x_{1}, \partial / \partial x_{2}\right)$. Similarly, for $\Gamma_{0}$ as part of the boundary of $\Omega_{\iota}^{\varepsilon}$, we define

$$
\mathcal{N}_{0, \iota}\left(x^{\prime}, x_{3}\right)=\frac{\iota\left(\partial_{1} h_{0}\left(x^{\prime}\right), \partial_{2} h_{0}\left(x^{\prime}\right),-1\right)}{\sqrt{1+\left|\nabla_{2} h_{0}\left(x^{\prime}\right)\right|^{2}}} .
$$

Then $\mathcal{N}_{\iota}$ and $\mathcal{N}_{0, \iota}$, for $\iota=+,-$, are $x_{3}$-independent vector fields on $\mathbb{T}^{2} \times \mathbb{R}$ that satisfy $\left.\mathcal{N}_{\iota}\right|_{\Gamma_{\iota}}=N_{\iota}$ and $\left.\mathcal{N}_{0, \iota}\right|_{\Gamma_{0}}=N_{\iota}$.

For specific tangential vector fields on $\partial \Omega^{\varepsilon}$ and their extensions, we define for $x=\left(x^{\prime}, x_{3}\right) \in$ $\mathbb{T}^{2} \times \mathbb{R}:$

$$
\begin{gathered}
\tau_{\iota}^{j}(x)=\left(e_{j}+\partial_{j} h_{\iota}\left(x^{\prime}\right) e_{3}\right) / \sqrt{1+\left|\partial_{j} h_{\iota}\left(x^{\prime}\right)\right|^{2}}, \quad \iota=-,+, \quad j=1,2, \\
\tau_{0}^{j}(x)=\left(e_{j}+\partial_{j} h_{0}\left(x^{\prime}\right) e_{3}\right) / \sqrt{1+\left|\partial_{j} h_{0}\left(x^{\prime}\right)\right|^{2}}, \quad j=1,2,
\end{gathered}
$$

where $\left\{e_{1}, e_{2}, e_{3}\right\}$ is the standard canonical basis of $\mathbb{R}^{3}$.

On each boundary $\Gamma_{\iota}$, for $\iota=+,-$, the two vectors $\tau_{\iota}^{1}$ and $\tau_{\iota}^{2}$ form a basis of the tangent space to $\Gamma_{\iota}$. Similarly, the two vectors $\tau_{0}^{1}$ and $\tau_{0}^{2}$ form a basis of the tangent space to $\Gamma_{0}$. They are all defined on $\mathbb{T}^{2} \times \mathbb{R}$ and are independent of $x_{3}$.

For these vector fields, we have the following estimates in $\mathbb{T}^{2} \times \mathbb{R}$ :

$$
\begin{gathered}
\left|\nabla \mathcal{N}_{\iota}\right|,\left|\nabla \mathcal{N}_{0, \iota}\right|,\left|\nabla \tau_{\iota}^{j}\right|,\left|\nabla \tau_{0}^{j}\right| \leq C \varepsilon \\
\left|e_{3}-\iota \mathcal{N}_{\iota}\right|,\left|e_{3}+\iota \mathcal{N}_{0, \iota}\right|,\left|e_{j}-\tau_{\iota}^{j}\right|,\left|e_{j}-\tau_{0}^{j}\right| \leq C \varepsilon
\end{gathered}
$$

for $\iota=+,-$ and $j=1,2$. For vector forms, we denote

$$
\begin{gathered}
\mathcal{N}=\left[\mathcal{N}_{+}, \mathcal{N}_{-}\right] \quad \text { and } \quad \mathcal{N}_{0}=\left[\mathcal{N}_{0,+}, \mathcal{N}_{0,-}\right], \\
\boldsymbol{\tau}^{j}=\left[\tau_{+}^{j}, \tau_{-}^{j}\right] \quad \text { and } \quad \boldsymbol{\tau}_{0}^{j}=\left[\tau_{0}^{j}, \tau_{0}^{j}\right] \quad \text { for } j=1,2 .
\end{gathered}
$$

For the orthonormal frames on the boundary, we define

$$
\tilde{\boldsymbol{\tau}}^{1}=\boldsymbol{\tau}^{1}, \tilde{\boldsymbol{\tau}}^{2}=\mathcal{N} \times \tilde{\boldsymbol{\tau}}^{1} \quad \text { and } \quad \tilde{\boldsymbol{\tau}}_{1}=\boldsymbol{\tau}_{0}^{1}, \tilde{\boldsymbol{\tau}}_{2}=\mathcal{N}_{\mathbf{0}} \times \tilde{\boldsymbol{\tau}}_{1}
$$

By (3.15) we have for $j=1,2$ that

$$
\left|\nabla \tilde{\boldsymbol{\tau}}^{j}\right|,\left|\nabla \tilde{\boldsymbol{\tau}}_{j}\right| \leq C \varepsilon \quad \text { in } \mathbb{T}^{2} \times \mathbb{R}
$$

As seen in $[7-9,15]$, boundary conditions affect estimates of different Sobolev norms. For instance, the following special Poincaré-like inequality for the third component of a vector field is proved in [9].

Lemma 3.3 ( [9], Lemma 4.7). If $\boldsymbol{u}$ is in $H^{1}\left(\Omega^{\varepsilon}\right)^{3}$ and satisfies (2.14), then

$$
\left[\left[\boldsymbol{u}_{3}\right]\right]_{L^{2}} \leq C \varepsilon[[\boldsymbol{u}]]_{H^{1}}
$$

where $C>0$ does not depend on $\varepsilon$.

For the partial derivative in $x_{3}$, we have the following version of Lemma 4.8 in [9]. 
Lemma 3.4. Let $\boldsymbol{u}$ belong to $H^{2}\left(\Omega^{\varepsilon}\right)^{3}$ and satisfy (2.10) and (2.11). Then

$$
\left[\left[\partial_{3} \boldsymbol{u}_{j}\right]\right]_{L^{2}} \leq C \varepsilon[[\boldsymbol{u}]]_{H^{2}}+C \sqrt{\varepsilon} \frac{\boldsymbol{\gamma}}{\boldsymbol{\nu}}[[\boldsymbol{u}]]_{L^{2}(\boldsymbol{\Gamma})}, \quad j=1,2,
$$

where $C>0$ is independent of $\varepsilon$.

Proof. The proof is the same as that of Lemma 4.2 in [8], using the boundary $\Gamma_{\iota}$ in place of $\Gamma_{0}$ there. Let $\iota=+,-$ and $j=1$. Using trace inequality (A.1), we have

$$
\left\|\partial_{3} u_{\iota, 1}\right\|_{L^{2}\left(\Omega_{\iota}\right)} \leq C \sqrt{\varepsilon}\left\|\partial_{3} u_{\iota, 1}\right\|_{L^{2}\left(\Gamma_{\iota}\right)}+C \varepsilon\left\|\partial_{3} \partial_{3} u_{\iota, 1}\right\|_{L^{2}\left(\Omega_{\iota}\right)} .
$$

On $\Gamma_{\iota}$ :

$$
\begin{aligned}
\partial_{3} u_{\iota, 1} & =\left(\nabla u_{\iota, 1}\right) \cdot\left(e_{3}-\iota N_{\iota}\right)+\iota\left(\nabla u_{\iota, 1}\right) \cdot N^{\iota} \\
& =\left(\nabla u_{\iota, 1}\right) \cdot\left(e_{3}-\iota N_{\iota}\right)+\iota\left(\left(\nabla u_{\iota}\right)^{*}\left(e_{1}-\tau_{\iota}^{1}\right)\right) \cdot N_{\iota}+\iota\left(\left(\nabla u_{\iota}\right)^{*} \tau_{\iota}^{1}\right) \cdot N_{\iota} \\
& =\left(\nabla u_{\iota, 1}\right) \cdot\left(e_{3}-\iota N_{\iota}\right)+\iota\left(\left(\nabla u_{\iota}\right)^{*}\left(e_{1}-\tau_{\iota}^{1}\right)\right) \cdot N_{\iota}+\iota\left(\left(\nabla u_{\iota}\right) N_{\iota}\right) \cdot \tau_{\iota}^{1} .
\end{aligned}
$$

Take $\tau$ in Lemma 3.1 to be $\tau^{1}$ defined by (3.18). Since $\boldsymbol{N}=\mathcal{N}$ on $\Gamma$, and we have from (3.2) that

$$
\left(\left(\nabla u_{\iota}\right) N_{\iota}\right) \cdot \tau_{\iota}^{1}=u_{\iota} \cdot\left(\left(\nabla \mathcal{N}_{\iota}\right) \tau_{\iota}^{1}\right)-2 \frac{\gamma_{\iota}}{\nu_{\iota}} u_{\iota} \cdot \tau_{\iota}^{1} \quad \text { on } \Gamma_{\iota} .
$$

Then thanks to (3.15) and (3.16), we obtain

$$
\left|\partial_{3} u_{\iota, 1}\right| \leq C \varepsilon\left|\nabla u_{\iota}\right|+C\left(\varepsilon+\frac{\gamma_{\iota}}{\nu_{\iota}}\right)\left|u_{\iota}\right| \leq C \varepsilon\left|\nabla u_{\iota}\right|+C \frac{\gamma_{\iota}}{\nu_{\iota}}\left|u_{\iota}\right| \text { on } \Gamma_{\iota} .
$$

Applying trace inequality (A.2) to $\left|\nabla u_{\iota}\right|$, we have

$$
\left\|\partial_{3} u_{\iota, 1}\right\|_{L^{2}\left(\Gamma_{\iota}\right)} \leq C \varepsilon\left(\frac{1}{\sqrt{\varepsilon}}\left\|u_{\iota}\right\|_{H^{1}\left(\Omega_{\iota}\right)}+\sqrt{\varepsilon}\left\|u_{\iota}\right\|_{H^{2}\left(\Omega_{\iota}\right)}\right)+\frac{\gamma_{\iota}}{\nu_{\iota}}\left\|u_{\iota}\right\|_{L^{2}\left(\Gamma_{\iota}\right)} .
$$

Together with (3.23),

$$
\left\|\partial_{3} u_{\iota, 1}\right\|_{L^{2}\left(\Omega_{\iota}\right)} \leq C \varepsilon\left\|u_{\iota}\right\|_{H^{2}\left(\Omega_{\iota}\right)}+C \sqrt{\varepsilon} \frac{\gamma_{\iota}}{\nu_{\iota}}\left\|u_{\iota}\right\|_{L^{2}\left(\Gamma_{\iota}\right)}, \quad \iota=+,-
$$

Therefore we obtain (3.22) for $j=1$. The case $j=2$ is similar.

Comparing (3.22) to the Poincaré inequality (A.1), we not only replace $\partial_{3} \boldsymbol{u}_{j}$ by $\boldsymbol{u}$ for the $L^{2}$-norm on $\Gamma$ but also gain the factor $\gamma / \nu \sim \varepsilon^{\delta}$.

Using estimates (3.15) and (3.20), we restate the integration by parts result of Lemma A.1 in [8] as follows.

Lemma 3.5 ( [8]). Let $\Sigma=\Gamma_{0}$, resp. $\Gamma_{+}, \Gamma_{-}$. Suppose $u(x)$ and $v(x)$ are two functions on $\Sigma$ and $\tau(x)$ is $\tau_{0}^{j}(x)$ or $\tilde{\tau}_{j \pm}(x)$, resp. $\tau_{+}^{j}(x)$ or $\tilde{\tau}_{+}^{j}(x), \tau_{-}^{j}(x)$ or $\tilde{\tau}_{-}^{j}(x)$, with $j=1$ or 2 . Then

$$
\int_{\Sigma} \frac{\partial u}{\partial \tau} \cdot v d \sigma=-\int_{\Sigma} \frac{\partial v}{\partial \tau} \cdot u d \sigma+O(\varepsilon) \int_{\Sigma}|u \cdot v| d \sigma
$$

Next, we compare the norm $\left\|\nabla^{2} \boldsymbol{u}\right\|_{L^{2}}$ with $\|\Delta \boldsymbol{u}\|_{L^{2}}$. We obtain a version of Lemma 5.4 in $[8]$. 
Lemma 3.6. There is $\varepsilon_{1} \in(0,1]$ such that if $\varepsilon \in\left(0, \varepsilon_{1}\right]$ and $\boldsymbol{u} \in H^{2}\left(\boldsymbol{\Omega}^{\varepsilon}\right)^{3}$ satisfies the boundary conditions (2.10), (2.11) and (2.12), (2.13), then

$$
\left\|\boldsymbol{\nu}^{1 / 2} \nabla^{2} \boldsymbol{u}\right\|_{L^{2}}^{2} \leq C\left(\left\|\boldsymbol{\nu}^{1 / 2} \Delta \boldsymbol{u}\right\|_{L^{2}}^{2}+|\boldsymbol{\nu}|_{\star}\|\boldsymbol{u}\|_{H^{1}}^{2}\right)
$$

and, consequently,

$$
\left\|\nabla^{2} \boldsymbol{u}\right\|_{\boldsymbol{L}^{2}} \leq C\left(\|\Delta \boldsymbol{u}\|_{L^{2}}+\|\boldsymbol{u}\|_{H^{1}}\right) .
$$

Proof. We follow the proof in Appendix A of [8]. Integration by parts twice gives

$$
\int_{\boldsymbol{\Omega}^{\varepsilon}}^{\star} \boldsymbol{\nu}\left|\nabla^{2} \boldsymbol{u}\right|^{2} d \boldsymbol{x}=\int_{\boldsymbol{\Omega}^{\varepsilon}}^{\star} \boldsymbol{\nu}|\Delta \boldsymbol{u}|^{2} d \boldsymbol{x}+I_{0},
$$

where

$$
I_{0}=\int_{\partial \boldsymbol{\Omega}^{\varepsilon}}^{\star} \boldsymbol{\nu}\left(\frac{1}{2} \frac{\partial|\nabla \boldsymbol{u}|^{2}}{\partial \boldsymbol{N}}-\frac{\partial \boldsymbol{u}}{\partial \boldsymbol{N}} \cdot \Delta \boldsymbol{u}\right) d \boldsymbol{\sigma} .
$$

We write $I_{0}=I_{0,+}+I_{0,-}+I_{0,0}$ where $I_{0,+}, I_{0,-}$ are the integrals on $\Gamma_{+}$and $\Gamma_{-}$, respectively, and

$$
I_{0,0}=\int_{\boldsymbol{\Gamma}_{0}}^{\star} \boldsymbol{\nu}\left(\frac{1}{2} \frac{\partial|\nabla \boldsymbol{u}|^{2}}{\partial \boldsymbol{N}}-\frac{\partial \boldsymbol{u}}{\partial \boldsymbol{N}} \cdot \Delta \boldsymbol{u}\right) d \boldsymbol{\sigma} .
$$

Exactly as in [8], by using extensions $\tilde{\boldsymbol{\tau}}^{1}, \tilde{\boldsymbol{\tau}}^{2}$ and $\mathcal{N}$ and working only on the separate components $\Gamma_{+}$and $\Gamma_{-}$of $\Gamma$, we have

$$
I_{0, \iota}=O(\varepsilon) \nu_{\iota} \int_{\Gamma_{\iota}}\left|\nabla u_{\iota}\right|^{2}+\left|u_{\iota}\right|^{2} d \sigma,
$$

hence

$$
I_{0,+}+I_{0,-}=O(\varepsilon) \int_{\Gamma}^{\star} \boldsymbol{\nu}\left(|\nabla \boldsymbol{u}|^{2}+|\boldsymbol{u}|^{2}\right) d \boldsymbol{\sigma} .
$$

(Many arguments leading to (3.30) are repeated below while we estimate $I_{0,0}$.)

Let $\tilde{\boldsymbol{\tau}}_{1}, \tilde{\boldsymbol{\tau}}_{2}$ be defined by (3.19). We have

$$
\left|\nabla \tilde{\boldsymbol{\tau}}_{1}\right|,\left|\nabla \tilde{\boldsymbol{\tau}}_{2}\right|,\left|\nabla^{2} \tilde{\boldsymbol{\tau}}_{1}\right|,\left|\nabla^{2} \tilde{\boldsymbol{\tau}}_{2}\right| \leq C \varepsilon \quad \text { on } \mathbb{T}^{2} \times \mathbb{R}
$$

In this proof, for calculations below, we use the extension $\mathcal{N}_{0}$ defined in (3.17) for $\boldsymbol{N}$ on $\Gamma_{0}$. Therefore, regarding estimates of derivatives of $\boldsymbol{N}$ on $\boldsymbol{\Gamma}_{0}$ we will use the following

$$
\left|\nabla \mathcal{N}_{0}\right|,\left|\nabla^{2} \mathcal{N}_{0}\right| \leq C \varepsilon \quad \text { on } \mathbb{T}^{2} \times \mathbb{R}
$$

Recall that $\left\{\tilde{\boldsymbol{\tau}}_{1}, \tilde{\boldsymbol{\tau}}_{2}, \mathcal{N}_{0}\right\}$ restricted to $\boldsymbol{\Gamma}_{0}$ forms an orthonormal frame on $\boldsymbol{\Gamma}_{0}$.

For $I_{0,0}$ we have similar calculations up to (A.9) of Appendix A in [8]:

$$
\begin{aligned}
I_{0,0}= & \int_{\boldsymbol{\Gamma}_{0}}^{\star} \boldsymbol{\nu} \frac{\partial^{2} \boldsymbol{u}}{\partial \tilde{\boldsymbol{\tau}}_{1} \partial \boldsymbol{N}} \cdot \frac{\partial \boldsymbol{u}}{\partial \tilde{\boldsymbol{\tau}}_{1}} d \boldsymbol{\sigma}+\int_{\boldsymbol{\Gamma}}^{\star} \boldsymbol{\nu} \frac{\partial^{2} \boldsymbol{u}}{\partial \tilde{\boldsymbol{\tau}}_{2} \partial \boldsymbol{N}} \cdot \frac{\partial \boldsymbol{u}}{\partial \tilde{\boldsymbol{\tau}}_{2}} d \boldsymbol{\sigma} \\
& -\int_{\boldsymbol{\Gamma}}^{\star} \boldsymbol{\nu}\left(\frac{\partial^{2} \boldsymbol{u}}{\partial \tilde{\boldsymbol{\tau}}_{1} \partial \tilde{\boldsymbol{\tau}}_{1}}+\frac{\partial^{2} \boldsymbol{u}}{\partial \tilde{\boldsymbol{\tau}}_{2} \partial \tilde{\boldsymbol{\tau}}_{2}}\right) \cdot \frac{\partial \boldsymbol{u}}{\partial \boldsymbol{N}} d \boldsymbol{\sigma}+J \\
= & I_{1}+I_{2}+I_{3}+J=\sum_{\iota=+,-}\left(I_{1, \iota}+I_{2, \iota}+I_{3, \iota}+J_{\iota}\right),
\end{aligned}
$$


where $\left|J_{\iota}\right|=O(\varepsilon) \nu_{\iota} \int_{\Gamma_{0}}\left|\nabla u_{\iota}\right|^{2} d \sigma$. Same as in [8],

$$
I_{3, \iota}=I_{1, \iota}+I_{2, \iota}+O(\varepsilon) \nu_{\iota} \int_{\Gamma_{0}}\left|\nabla u_{\iota}\right|^{2} d \sigma, \quad \iota=+,-
$$

Therefore

$$
I_{0,0}=2\left(I_{1}+I_{2}\right)+O(\varepsilon) \int_{\boldsymbol{\Gamma}_{0}}^{\star} \boldsymbol{\nu}|\nabla \boldsymbol{u}|^{2} d \boldsymbol{\sigma} .
$$

Combining (3.34) with (3.31) gives

$$
I_{0}=2\left(I_{1}+I_{2}\right)+O(\varepsilon) \int_{\partial \boldsymbol{\Omega}^{\varepsilon}}^{\star} \boldsymbol{\nu}\left(|\nabla \boldsymbol{u}|^{2}+|\boldsymbol{u}|^{2}\right) d \boldsymbol{\sigma} .
$$

We now estimate $I_{1}$ and $I_{2}$. For $\boldsymbol{\tau}, \boldsymbol{\tau}^{\prime} \in\left\{\tilde{\boldsymbol{\tau}}_{1}, \tilde{\boldsymbol{\tau}}_{2}\right\}$, taking the derivative $\partial / \partial \boldsymbol{\tau}^{\prime}$ of identity (3.5) on $\Gamma_{0}$ gives

$$
\begin{aligned}
\frac{\partial^{2} \boldsymbol{u}}{\partial \boldsymbol{\tau}^{\prime} \partial \boldsymbol{N}} \cdot \boldsymbol{\tau}+\frac{\partial \boldsymbol{u}}{\partial \boldsymbol{N}} \cdot \frac{\partial \boldsymbol{N}}{\partial \boldsymbol{\tau}^{\prime}}= & \left(\frac{\partial \boldsymbol{u}}{\partial \boldsymbol{\tau}^{\prime}} \cdot \frac{\partial \boldsymbol{N}}{\partial \boldsymbol{\tau}}+\boldsymbol{u} \cdot \frac{\partial^{2} \boldsymbol{N}}{\partial \boldsymbol{\tau}^{\prime} \partial \boldsymbol{\tau}}\right) \\
& -2 \frac{\gamma_{0}}{\boldsymbol{\nu}} \frac{\partial(\boldsymbol{u}-S \boldsymbol{u})}{\partial \boldsymbol{\tau}^{\prime}} \cdot \boldsymbol{\tau}-2 \frac{\gamma_{0}}{\boldsymbol{\nu}}(\boldsymbol{u}-S \boldsymbol{u}) \cdot \frac{\partial \boldsymbol{\tau}}{\partial \boldsymbol{\tau}^{\prime}}
\end{aligned}
$$

Using (3.33) to estimate the first and second order derivatives of $\boldsymbol{N}$ on $\boldsymbol{\Gamma}_{0}$ we can bound the second term on the left-hand side and the first two terms on the right-hand side by $O(\varepsilon)(|\nabla \boldsymbol{u}|+$ $|\boldsymbol{u}|)$. Using properties (2.17), (2.18) and (3.32), we bound the last term of (3.36) by $O(\varepsilon)|\boldsymbol{u}|_{\star}$. Thus, we have

$$
\frac{\partial^{2} \boldsymbol{u}}{\partial \boldsymbol{\tau}^{\prime} \partial \boldsymbol{N}} \cdot \boldsymbol{\tau}=-2 \frac{\gamma_{0}}{\boldsymbol{\nu}} \frac{\partial(\boldsymbol{u}-S \boldsymbol{u})}{\partial \boldsymbol{\tau}^{\prime}} \cdot \boldsymbol{\tau}+O(\varepsilon)\left(|\nabla \boldsymbol{u}|+|\boldsymbol{u}|_{\star}\right)
$$

For the integrand of $I_{1}$, writing the dot product in the orthonormal basis $\left\{\tilde{\boldsymbol{\tau}}_{1}, \tilde{\boldsymbol{\tau}}_{2}, \boldsymbol{N}\right\}$ gives

$$
\begin{aligned}
& \boldsymbol{\nu} \frac{\partial^{2} \boldsymbol{u}}{\partial \tilde{\boldsymbol{\tau}}_{1} \partial \boldsymbol{N}} \cdot \frac{\partial \boldsymbol{u}}{\partial \tilde{\boldsymbol{\tau}}_{1}}=\boldsymbol{\nu}\left(\frac{\partial^{2} \boldsymbol{u}}{\partial \tilde{\boldsymbol{\tau}}_{1} \partial \boldsymbol{N}} \cdot \tilde{\boldsymbol{\tau}}_{1}\right)\left(\frac{\partial \boldsymbol{u}}{\partial \tilde{\boldsymbol{\tau}}_{1}} \cdot \tilde{\boldsymbol{\tau}}_{1}\right) \\
& +\boldsymbol{\nu}\left(\frac{\partial^{2} \boldsymbol{u}}{\partial \tilde{\boldsymbol{\tau}}_{1} \partial \boldsymbol{N}} \cdot \tilde{\boldsymbol{\tau}}_{2}\right)\left(\frac{\partial \boldsymbol{u}}{\partial \tilde{\boldsymbol{\tau}}_{1}} \cdot \tilde{\boldsymbol{\tau}}_{2}\right)+\boldsymbol{\nu}\left(\frac{\partial^{2} \boldsymbol{u}}{\partial \tilde{\boldsymbol{\tau}}_{1} \partial \boldsymbol{N}} \cdot \boldsymbol{N}\right)\left(\frac{\partial \boldsymbol{u}}{\partial \tilde{\boldsymbol{\tau}}_{1}} \cdot \boldsymbol{N}\right) .
\end{aligned}
$$

By (3.37), the first term on the right-hand side of (3.38) is

$$
\begin{aligned}
& \boldsymbol{\nu}\left(\frac{\partial^{2} \boldsymbol{u}}{\partial \tilde{\boldsymbol{\tau}}_{1} \partial \boldsymbol{N}} \cdot \tilde{\boldsymbol{\tau}}_{1}\right)\left(\frac{\partial \boldsymbol{u}}{\partial \tilde{\boldsymbol{\tau}}_{1}} \cdot \tilde{\boldsymbol{\tau}}_{1}\right) \\
& =\left\{-2 \gamma_{0}\left(\frac{\partial(\boldsymbol{u}-S \boldsymbol{u})}{\partial \tilde{\boldsymbol{\tau}}_{1}} \cdot \tilde{\boldsymbol{\tau}}_{1}\right)+O(\varepsilon) \boldsymbol{\nu}\left(|\nabla \boldsymbol{u}|+|\boldsymbol{u}|_{\star}\right)\right\}\left(\frac{\partial \boldsymbol{u}}{\partial \tilde{\boldsymbol{\tau}}_{1}} \cdot \tilde{\boldsymbol{\tau}}_{1}\right) \\
& =-2 \gamma_{0}\left(\frac{\partial(\boldsymbol{u}-S \boldsymbol{u})}{\partial \tilde{\boldsymbol{\tau}}_{1}} \cdot \tilde{\boldsymbol{\tau}}_{1}\right)\left(\frac{\partial \boldsymbol{u}}{\partial \tilde{\boldsymbol{\tau}}_{1}} \cdot \tilde{\boldsymbol{\tau}}_{1}\right)+O(\varepsilon) \boldsymbol{\nu}\left(|\nabla \boldsymbol{u}|^{2}+|\boldsymbol{u}|_{\star}|\nabla \boldsymbol{u}|\right) .
\end{aligned}
$$


The second term on the right-hand side of (3.38) is treated similarly. Also, we use (3.4) for the last factor of (3.38). We obtain

$$
\begin{aligned}
\boldsymbol{\nu} & \frac{\partial^{2} \boldsymbol{u}}{\partial \tilde{\boldsymbol{\tau}}_{1} \partial \boldsymbol{N}} \cdot \frac{\partial \boldsymbol{u}}{\partial \tilde{\boldsymbol{\tau}}_{1}} \\
= & -2 \gamma_{0}\left(\frac{\partial(\boldsymbol{u}-S \boldsymbol{u})}{\partial \tilde{\boldsymbol{\tau}}_{1}} \cdot \tilde{\boldsymbol{\tau}}_{1}\right)\left(\frac{\partial \boldsymbol{u}}{\partial \tilde{\boldsymbol{\tau}}_{1}} \cdot \tilde{\boldsymbol{\tau}}_{1}\right)-2 \gamma_{0}\left(\frac{\partial(\boldsymbol{u}-S \boldsymbol{u})}{\partial \tilde{\boldsymbol{\tau}}_{1}} \cdot \tilde{\boldsymbol{\tau}}_{2}\right)\left(\frac{\partial \boldsymbol{u}}{\partial \tilde{\boldsymbol{\tau}}_{1}} \cdot \tilde{\boldsymbol{\tau}}_{2}\right) \\
& -\boldsymbol{\nu}\left(\frac{\partial^{2} \boldsymbol{u}}{\partial \tilde{\boldsymbol{\tau}}_{1} \partial \boldsymbol{N}} \cdot \boldsymbol{N}\right)\left(\frac{\partial \boldsymbol{N}}{\partial \tilde{\boldsymbol{\tau}}_{1}} \cdot \boldsymbol{u}\right)+O(\varepsilon) \boldsymbol{\nu}\left(|\nabla \boldsymbol{u}|^{2}+|\boldsymbol{u}|_{\star}|\nabla \boldsymbol{u}|\right) .
\end{aligned}
$$

We have on $\boldsymbol{\Gamma}_{0}$ that $S \boldsymbol{N}=-\boldsymbol{N}, S \tilde{\boldsymbol{\tau}}_{1}=\tilde{\boldsymbol{\tau}}_{1}$, hence $S \tilde{\boldsymbol{\tau}}_{2}=-\tilde{\boldsymbol{\tau}}_{2}$. Using these properties and writing the following sums explicitly, we obtain

$$
\begin{aligned}
& 2 \sum_{\iota=+,-}\left[\left(\frac{\partial(\boldsymbol{u}-S \boldsymbol{u})}{\partial \tilde{\boldsymbol{\tau}}_{1}} \cdot \tilde{\boldsymbol{\tau}}_{1}\right)\left(\frac{\partial \boldsymbol{u}}{\partial \tilde{\boldsymbol{\tau}}_{1}} \cdot \tilde{\boldsymbol{\tau}}_{1}\right)\right]_{\iota}=\left|\frac{\partial(\boldsymbol{u}-S \boldsymbol{u})}{\partial \tilde{\boldsymbol{\tau}}_{1}} \cdot \tilde{\boldsymbol{\tau}}_{1}\right|_{\star}^{2} \geq 0, \\
& 2 \sum_{\iota=+,-}\left[\left(\frac{\partial(\boldsymbol{u}-S \boldsymbol{u})}{\partial \tilde{\boldsymbol{\tau}}_{1}} \cdot \tilde{\boldsymbol{\tau}}_{2}\right)\left(\frac{\partial \boldsymbol{u}}{\partial \tilde{\boldsymbol{\tau}}_{1}} \cdot \tilde{\boldsymbol{\tau}}_{2}\right)\right]_{\iota}=\left|\frac{\partial(\boldsymbol{u}-S \boldsymbol{u})}{\partial \tilde{\boldsymbol{\tau}}_{1}} \cdot \tilde{\boldsymbol{\tau}}_{2}\right|_{\star}^{2} \geq 0 .
\end{aligned}
$$

Therefore, summing the + and - components of (3.39) and integrating over $\Gamma_{0}$ yields

$$
\begin{aligned}
I_{1} & =\sum_{\iota=+,-} \int_{\Gamma_{0}} \nu_{\iota}\left[\frac{\partial^{2} \boldsymbol{u}}{\partial \tilde{\boldsymbol{\tau}}_{1} \partial \boldsymbol{N}} \cdot \frac{\partial \boldsymbol{u}}{\partial \tilde{\boldsymbol{\tau}}_{1}}\right]_{\iota} d \sigma \\
& \leq-\int_{\boldsymbol{\Gamma}_{0}}^{\star} \boldsymbol{\nu} \frac{\partial^{2} \boldsymbol{u}}{\partial \tilde{\boldsymbol{\tau}}_{1} \partial \boldsymbol{N}} \cdot\left\{\boldsymbol{N}\left(\boldsymbol{u} \cdot \frac{\partial \boldsymbol{N}}{\partial \tilde{\boldsymbol{\tau}}_{1}}\right)\right\} d \boldsymbol{\sigma}+O(\varepsilon)|\boldsymbol{\nu}|_{\star} \int_{\Gamma_{0}}\left(|\nabla u|_{\star}^{2}+|u|_{\star}|\nabla u|_{\star}\right) d \sigma .
\end{aligned}
$$

We apply Lemma 3.5 to the middle integral of (3.40):

$$
\begin{aligned}
& -\int_{\boldsymbol{\Gamma}_{0}}^{\star} \boldsymbol{\nu} \frac{\partial}{\partial \tilde{\boldsymbol{\tau}}_{1}}\left(\frac{\partial \boldsymbol{u}}{\partial \boldsymbol{N}}\right) \cdot\left\{\boldsymbol{N}\left(\boldsymbol{u} \cdot \frac{\partial \boldsymbol{N}}{\partial \tilde{\boldsymbol{\tau}}_{1}}\right)\right\} d \boldsymbol{\sigma} \\
& =\int_{\boldsymbol{\Gamma}_{0}}^{\star} \boldsymbol{\nu} \frac{\partial \boldsymbol{u}}{\partial \boldsymbol{N}} \cdot \frac{\partial}{\partial \tilde{\boldsymbol{\tau}}_{1}}\left\{\boldsymbol{N}\left(\boldsymbol{u} \cdot \frac{\partial \boldsymbol{N}}{\partial \tilde{\boldsymbol{\tau}}_{1}}\right)\right\} d \boldsymbol{\sigma}+O(\varepsilon) \int_{\boldsymbol{\Gamma}_{0}}^{\star} \boldsymbol{\nu}\left|\frac{\partial \boldsymbol{u}}{\partial \boldsymbol{N}} \cdot\left\{\boldsymbol{N}\left(\boldsymbol{u} \cdot \frac{\partial \boldsymbol{N}}{\partial \tilde{\boldsymbol{\tau}}_{1}}\right)\right\}\right| d \boldsymbol{\sigma} \\
& =O(\varepsilon) \int_{\boldsymbol{\Gamma}_{0}}^{\star} \boldsymbol{\nu}|\nabla \boldsymbol{u}|(|\nabla \boldsymbol{u}|+|\boldsymbol{u}|) d \boldsymbol{\sigma}+O(\varepsilon) \int_{\boldsymbol{\Gamma}_{0}}^{\star} \boldsymbol{\nu}|\nabla \boldsymbol{u} \| \boldsymbol{u}| d \boldsymbol{\sigma} .
\end{aligned}
$$

(For the last identity, we use (3.33) again to estimate $\partial \boldsymbol{N} / \partial \tilde{\boldsymbol{\tau}}_{1}$.) Therefore, it follows (3.40) and Cauchy's inequality that

$$
I_{1} \leq C \varepsilon \int_{\Gamma_{0}}|\boldsymbol{\nu}|_{\star}\left(|\nabla \boldsymbol{u}|_{\star}^{2}+|\boldsymbol{u}|_{\star}^{2}\right) d \sigma \leq C \varepsilon|\boldsymbol{\nu}|_{\star} \int_{\Gamma_{0}}^{\star}\left(|\nabla \boldsymbol{u}|^{2}+|\boldsymbol{u}|^{2}\right) d \boldsymbol{\sigma} .
$$

Similarly,

$$
I_{2} \leq C \varepsilon|\boldsymbol{\nu}|_{\star} \int_{\Gamma_{0}}^{\star}\left(|\nabla \boldsymbol{u}|^{2}+|\boldsymbol{u}|^{2}\right) d \boldsymbol{\sigma}
$$

Then combining (3.35) with above estimates of $I_{1}, I_{2}$ and using the trace inequality (A.2) we have

$$
I_{0} \leq C \varepsilon|\boldsymbol{\nu}|_{\star} \int_{\partial \boldsymbol{\Omega}^{\varepsilon}}^{\star}\left(|\nabla \boldsymbol{u}|^{2}+|\boldsymbol{u}|^{2}\right) d \boldsymbol{\sigma} \leq C|\boldsymbol{\nu}|_{\star}\left(\|\boldsymbol{u}\|_{\boldsymbol{H}^{1}}^{2}+\varepsilon^{2}\left\|\nabla^{2} \boldsymbol{u}\right\|_{\boldsymbol{L}^{2}}^{2}\right) .
$$


By (2.16), it follows (3.27) and (3.41) that

$$
\left\|\boldsymbol{\nu}^{1 / 2} \nabla^{2} \boldsymbol{u}\right\|_{L^{2}}^{2} \leq\left\|\boldsymbol{\nu}^{1 / 2} \Delta \boldsymbol{u}\right\|_{L^{2}}^{2}+C|\boldsymbol{\nu}|_{\star}\|\boldsymbol{u}\|_{\boldsymbol{H}^{1}}^{2}+C \varepsilon^{2}\left\|\boldsymbol{\nu}^{1 / 2} \nabla^{2} \boldsymbol{u}\right\|_{\boldsymbol{L}^{2}}^{2} .
$$

For $\varepsilon>0$ sufficiently small, the term $C \varepsilon^{2}\left\|\nu^{1 / 2} \nabla^{2} \boldsymbol{u}\right\|_{L^{2}}^{2}$ on the right-hand side of (3.42) can be absorbed into the left-hand side, hence we obtain (3.25). Then by (2.16) again, inequality (3.26) follows (3.25). The proof is complete.

For further estimates in sections 4 and 5 below, we need to apply (3.3) and (3.6) with the same extension $\boldsymbol{n}$. The above extensions $\mathcal{N}$ and $\mathcal{N}_{0}$, however, cannot be matched on $\Gamma$ and $\Gamma_{0}$ in general. Therefore we introduce new extension vector fields in the following.

For $x=\left(x^{\prime}, x_{3}\right) \in \mathbb{T}^{2} \times \mathbb{R}$, define

$$
\tilde{\mathcal{N}}_{\iota}(x)=\frac{x_{3}-h_{0}\left(x^{\prime}\right)}{\iota \varepsilon g_{\iota}\left(x^{\prime}\right)} \mathcal{N}_{\iota}(x)-\frac{h_{\iota}\left(x^{\prime}\right)-x_{3}}{\iota \varepsilon g_{\iota}\left(x^{\prime}\right)} \mathcal{N}_{0, \iota}(x)
$$

and its related function

$$
\tilde{\tilde{\mathcal{N}}}_{\iota}(x)=\frac{x_{3}-h_{0}\left(x^{\prime}\right)}{\iota \varepsilon g_{\iota}\left(x^{\prime}\right)} \cdot \frac{\gamma_{\iota}}{\nu_{\iota}} \mathcal{N}_{\iota}(x)+\frac{h_{\iota}\left(x^{\prime}\right)-x_{3}}{\iota \varepsilon g_{\iota}\left(x^{\prime}\right)} \cdot \frac{\gamma_{0}}{\nu_{\iota}} \mathcal{N}_{0, \iota}(x) .
$$

Then $\left.\tilde{\mathcal{N}}_{\iota}\right|_{\Gamma_{\iota}}=N_{\iota}$ and $\left.\tilde{\mathcal{N}}_{\iota}\right|_{\Gamma_{0}}=-N_{\iota}$. We have on $\mathbb{T}^{2} \times \mathbb{R}$ :

$$
\begin{gathered}
\left|\tilde{\mathcal{N}}_{\iota, j}\right|,\left|\partial_{j} \tilde{\mathcal{N}}_{\iota}\right| \leq C \varepsilon, \quad \text { for } j=1,2, \quad\left|\tilde{\mathcal{N}}_{\iota, 3}\right|,\left|\partial_{3} \tilde{\mathcal{N}}_{\iota}\right|,\left|\nabla^{2} \tilde{\mathcal{N}}_{\iota}\right| \leq C, \\
\left|\tilde{\mathcal{N}}_{\iota}+\tilde{\mathcal{N}}_{0, \iota}\right| \leq C \varepsilon, \quad\left|\tilde{\mathcal{N}}_{\iota}-\tilde{\mathcal{N}}_{0, \iota}\right| \leq C .
\end{gathered}
$$

Also, $\left.\tilde{\tilde{\mathcal{N}}}_{\iota}\right|_{\Gamma_{\iota}}=\frac{\gamma_{\iota}}{\nu_{\iota}} N_{\iota}$ and $\left.\tilde{\tilde{\mathcal{N}}}_{\iota}\right|_{\Gamma_{0}}=\frac{\gamma_{0}}{\nu_{\iota}} N_{\iota}$ and

$$
\left|\tilde{\tilde{\mathcal{N}}}_{\iota}\right| \leq C \frac{\gamma_{0}+\gamma_{\iota}}{\nu_{\iota}} \leq C \varepsilon^{\delta}, \quad\left|\nabla_{2} \tilde{\tilde{\mathcal{N}}}_{\iota}\right| \leq C \varepsilon^{\delta}, \quad\left|\partial_{3} \tilde{\tilde{\mathcal{N}}}_{\iota}\right| \leq C \varepsilon^{\delta-1} \quad \text { on } \mathbb{T}^{2} \times \mathbb{R} .
$$

(Above, we used the facts $0<\delta \leq \delta_{0}$ and $0<\varepsilon \leq 1$.) Therefore

$$
\left|\nabla \tilde{\tilde{\mathcal{N}}}_{\iota}\right| \leq C \varepsilon^{\delta-1} \quad \text { on } \mathbb{T}^{2} \times \mathbb{R}
$$

Let $\widetilde{\mathcal{N}}=\left[\tilde{\mathcal{N}}_{+}, \tilde{\mathcal{N}}_{-}\right]$and $\widetilde{\widetilde{\mathcal{N}}}=\left[\tilde{\tilde{\mathcal{N}}}_{+}, \tilde{\tilde{\mathcal{N}}}_{-}\right]$. Then $\widetilde{\mathcal{N}}$ is an extension of the upward/downward normal vectors on the boundary $\partial \boldsymbol{\Omega}^{\varepsilon}$.

Lemma 3.7. Suppose $\boldsymbol{u} \in H^{2}\left(\Omega^{\varepsilon}\right)^{3}$ satisfies the boundary conditions (2.10), (2.11) and (2.12), (2.13). Then

$$
\begin{gathered}
\boldsymbol{N} \times(\nabla \times \boldsymbol{u})=\boldsymbol{N} \times G(\boldsymbol{u}) \quad \text { on } \quad \boldsymbol{\Gamma}, \\
\boldsymbol{N} \times(\nabla \times \boldsymbol{u})=\boldsymbol{N} \times G(\boldsymbol{u})-2 \frac{\gamma_{0}}{\nu} S \boldsymbol{u} \quad \text { on } \boldsymbol{\Gamma}_{0},
\end{gathered}
$$

where $G(\boldsymbol{u})$ is a vector field on $\Omega^{\varepsilon}$ defined by (3.53) below and satisfies the following estimates in $\Omega^{\varepsilon}$ :

$$
\begin{gathered}
|G(\boldsymbol{u})| \leq C \varepsilon^{\delta}|\boldsymbol{u}| \\
|\nabla G(\boldsymbol{u})| \leq C\left(\varepsilon^{\delta}|\nabla \boldsymbol{u}|+\varepsilon^{\delta-1}|\boldsymbol{u}|\right) .
\end{gathered}
$$


Proof. Using the identity (3.3) and (3.6) with $\boldsymbol{n}=\widetilde{\mathcal{N}}$, we have (3.49) and (3.50), where the vector field $G(\boldsymbol{u})$ is defined on $\Omega^{\varepsilon}$ by

$$
G(\boldsymbol{u})=G^{(1)}(\boldsymbol{u})-G^{(2)}(\boldsymbol{u}),
$$

with

$$
G^{(1)}(\boldsymbol{u})=2 \widetilde{\mathcal{N}} \times\left[(\nabla \widetilde{\mathcal{N}})^{*} \boldsymbol{u}\right] \quad \text { and } \quad G^{(2)}(\boldsymbol{u})=2 \widetilde{\widetilde{\mathcal{N}}} \times \boldsymbol{u} .
$$

As in $[9,15]$, we have

$$
\left|G^{(1)}(\boldsymbol{u})\right| \leq C \varepsilon|\boldsymbol{u}| \quad \text { and } \quad\left|\nabla G^{(1)}(\boldsymbol{u})\right| \leq C \varepsilon|\nabla \boldsymbol{u}|+C|\boldsymbol{u}| \quad \text { on } \Omega^{\varepsilon} .
$$

By (3.47) and (3.48), one obtains

$$
\begin{gathered}
\left|G^{(2)}(\boldsymbol{u})\right| \leq 2|\widetilde{\widetilde{\mathcal{N}}}||\boldsymbol{u}| \leq C \varepsilon^{\delta}|\boldsymbol{u}|, \\
\left|\nabla G^{(2)}(\boldsymbol{u})\right| \leq C(|\nabla \boldsymbol{u}||\widetilde{\widetilde{\mathcal{N}}}|+|\boldsymbol{u}||\nabla \widetilde{\widetilde{\mathcal{N}}}|) \leq C\left(\varepsilon^{\delta}|\nabla \boldsymbol{u}|+\varepsilon^{\delta-1}|\boldsymbol{u}|\right) .
\end{gathered}
$$

Combining (3.53), (3.55), (3.56) and (3.57) yields (3.51) and (3.52).

The NSE has an important curl structure. The following lemma connects this curl structure to the boundary conditions, especially on the interface.

Lemma 3.8. If $\boldsymbol{u} \in H^{2}\left(\boldsymbol{\Omega}^{\varepsilon}\right)^{3}$ satisfies (2.10), (2.11) and (2.12), (2.13), and $\boldsymbol{\Phi} \in H^{1}\left(\boldsymbol{\Omega}^{\varepsilon}\right)^{3}$, then

$$
\begin{aligned}
\int_{\boldsymbol{\Omega}^{\varepsilon}}^{\star} \boldsymbol{\nu}(\nabla \times(\nabla \times \boldsymbol{u})) \cdot \boldsymbol{\Phi} d \boldsymbol{x}= & \int_{\boldsymbol{\Omega}^{\varepsilon}}^{\star} \boldsymbol{\nu}(\nabla \times \boldsymbol{\Phi}) \cdot(\nabla \times \boldsymbol{u}-G(\boldsymbol{u})) d \boldsymbol{x} \\
& +\int_{\boldsymbol{\Omega}^{\varepsilon}}^{\star} \boldsymbol{\nu} \boldsymbol{\Phi} \cdot(\nabla \times G(\boldsymbol{u})) d \boldsymbol{x}-2 \gamma_{0} \int_{\boldsymbol{\Gamma}_{0}}^{\star} \boldsymbol{u} \cdot S \boldsymbol{\Phi} d \boldsymbol{\sigma},
\end{aligned}
$$

where $G(\boldsymbol{u})$ is defined in Lemma 3.7.

Proof. Let $\boldsymbol{\omega}=\nabla \times \boldsymbol{u}$, one has

$$
\int_{\boldsymbol{\Omega}^{\varepsilon}}^{\star} \boldsymbol{\nu}(\nabla \times \boldsymbol{\omega}) \cdot \boldsymbol{\Phi} d \boldsymbol{x}=\int_{\boldsymbol{\Omega}^{\varepsilon}}^{\star} \boldsymbol{\nu} \boldsymbol{\omega} \cdot(\nabla \times \boldsymbol{\Phi}) d \boldsymbol{x}+\int_{\partial \boldsymbol{\Omega}^{\varepsilon}}^{\star} \boldsymbol{\nu}(\boldsymbol{N} \times \boldsymbol{\omega}) \cdot \boldsymbol{\Phi} d \boldsymbol{\sigma} .
$$

For the boundary integral, by (3.49) and (3.50),

$$
\begin{aligned}
& \int_{\partial \boldsymbol{\Omega}^{\varepsilon}}^{\star} \boldsymbol{\nu}(\boldsymbol{N} \times \boldsymbol{\omega}) \cdot \boldsymbol{\Phi} d \boldsymbol{\sigma}=\int_{\boldsymbol{\Gamma}}^{\star} \boldsymbol{\nu}(\boldsymbol{N} \times \boldsymbol{\omega}) \cdot \boldsymbol{\Phi} d \boldsymbol{\sigma}+\int_{\boldsymbol{\Gamma}_{0}}^{\star} \boldsymbol{\nu}(\boldsymbol{N} \times \boldsymbol{\omega}) \cdot \boldsymbol{\Phi} d \boldsymbol{\sigma} \\
& =\int_{\boldsymbol{\Gamma} \cup \boldsymbol{\Gamma}_{0}}^{\star} \boldsymbol{\nu}(\boldsymbol{N} \times G(\boldsymbol{u})) \cdot \boldsymbol{\Phi} d \boldsymbol{\sigma}-2 \gamma_{0} \int_{\boldsymbol{\Gamma}_{0}}^{\star} S \boldsymbol{u} \cdot \boldsymbol{\Phi} d \boldsymbol{\sigma} \\
& =-\int_{\partial \boldsymbol{\Omega}^{\varepsilon}}^{\star} \boldsymbol{\nu}(\boldsymbol{\Phi} \times G(\boldsymbol{u})) \cdot \boldsymbol{N} d \boldsymbol{\sigma}-2 \gamma_{0} \int_{\boldsymbol{\Gamma}_{0}}^{\star} \boldsymbol{u} \cdot S \boldsymbol{\Phi} d \boldsymbol{\sigma} .
\end{aligned}
$$

Applying the Divergence Theorem to the integral on $\partial \boldsymbol{\Omega}^{\varepsilon}$ gives

$$
\begin{aligned}
& \int_{\partial \boldsymbol{\Omega}^{\varepsilon}}^{\star} \boldsymbol{\nu}(\boldsymbol{\Phi} \times G(\boldsymbol{u})) \cdot \boldsymbol{N} d \boldsymbol{\sigma}=\int_{\boldsymbol{\Omega}^{\varepsilon}}^{\star} \boldsymbol{\nu} \nabla \cdot(\boldsymbol{\Phi} \times G(\boldsymbol{u})) d \boldsymbol{x} \\
& =\int_{\boldsymbol{\Omega}^{\varepsilon}}^{\star} \boldsymbol{\nu}(\nabla \times \boldsymbol{\Phi}) \cdot G(\boldsymbol{u})-\boldsymbol{\nu} \boldsymbol{\Phi} \cdot(\nabla \times G(\boldsymbol{u})) d \boldsymbol{x} .
\end{aligned}
$$


Combining the above calculations, we obtain (3.58).

Comparing Lemma 3.8 with its counterpart for a single layer domain - Lemma 5.2 in [8] the additional boundary integrals on $\Gamma_{0}$ in (3.58) shows the interaction between $u_{\iota}$ and $\Phi_{-\iota}$ on $\Gamma_{0}$ for $\iota=+,-$.

\section{STOKES AND AVERAGING OPERATORS}

4.1. Stokes operator and functional form of NSE. For $\boldsymbol{u}=\left[u_{+}, u_{-}\right] \in H^{2}\left(\boldsymbol{\Omega}^{\varepsilon}\right)^{3}$ and $\boldsymbol{v}=$ $\left[v_{+}, v_{-}\right] \in H^{1}\left(\Omega^{\varepsilon}\right)^{3}$, we have the following Green's formula (c.f. $\left.[15,25]\right)$ :

$$
\begin{aligned}
\int_{\boldsymbol{\Omega}^{\varepsilon}} \boldsymbol{\nu} \Delta \boldsymbol{u} \cdot \boldsymbol{v} d \boldsymbol{x}=\int_{\boldsymbol{\Omega}^{\varepsilon}}-2 \boldsymbol{\nu}(D \boldsymbol{u}: & D \boldsymbol{v})+\boldsymbol{\nu}(\nabla \cdot \boldsymbol{u})(\nabla \cdot \boldsymbol{v}) d \boldsymbol{x} \\
& +\int_{\partial \boldsymbol{\Omega}^{\varepsilon}}\{2 \boldsymbol{\nu}((D \boldsymbol{u}) \boldsymbol{N}) \cdot \boldsymbol{v}-\boldsymbol{\nu}(\nabla \cdot \boldsymbol{u})(\boldsymbol{v} \cdot \boldsymbol{N})\} d \boldsymbol{\sigma},
\end{aligned}
$$

where the symbol : denotes the usual scalar product of two matrices.

If $\boldsymbol{u}$ is divergence-free and satisfies the boundary conditions (2.10), (2.11) and (2.12), (2.13), and $\boldsymbol{v}$ is tangential to the boundary $\partial \boldsymbol{\Omega}^{\varepsilon}$ then

$$
\begin{aligned}
-\int_{\boldsymbol{\Omega}^{\varepsilon}} \boldsymbol{\nu} \Delta \boldsymbol{u} \cdot \boldsymbol{v} d \boldsymbol{x}= & 2 \int_{\boldsymbol{\Omega}^{\varepsilon}} \boldsymbol{\nu}(D \boldsymbol{u}: D \boldsymbol{v}) d \boldsymbol{x} \\
& +2 \int_{\boldsymbol{\Gamma}} \boldsymbol{\gamma} \boldsymbol{u} \cdot \boldsymbol{v} d \boldsymbol{\sigma}+2 \gamma_{0} \int_{\boldsymbol{\Gamma}_{0}}(\boldsymbol{u}-S \boldsymbol{u}) \cdot \boldsymbol{v} d \boldsymbol{\sigma} .
\end{aligned}
$$

Consequently, we obtain

$$
-\int_{\boldsymbol{\Omega}^{\varepsilon}}^{\star} \boldsymbol{\nu} \Delta \boldsymbol{u} \cdot \boldsymbol{v} d \boldsymbol{x}=E(\boldsymbol{u}, \boldsymbol{v})
$$

where

$$
E(\boldsymbol{u}, \boldsymbol{v})=2 \int_{\boldsymbol{\Omega}^{\varepsilon}}^{\star} \boldsymbol{\nu} D \boldsymbol{u}: D \boldsymbol{v} d \boldsymbol{x}+2 \int_{\boldsymbol{\Gamma}}^{\star} \boldsymbol{\gamma} \boldsymbol{u} \cdot \boldsymbol{v} d \boldsymbol{\sigma}+\gamma_{0} \int_{\boldsymbol{\Gamma}_{0}}^{\star}(\boldsymbol{u}-S \boldsymbol{u}) \cdot(\boldsymbol{v}-S \boldsymbol{v}) d \boldsymbol{\sigma} .
$$

In case $\boldsymbol{u}=\boldsymbol{v}$ we have

$$
-\int_{\boldsymbol{\Omega}^{\varepsilon}}^{\star} \boldsymbol{\nu} \Delta \boldsymbol{u} \cdot \boldsymbol{u} d \boldsymbol{x}=E(\boldsymbol{u})
$$

where $E(\boldsymbol{u})$ is defined by

$$
E(\boldsymbol{u})=E(\boldsymbol{u}, \boldsymbol{u})=2 \int_{\boldsymbol{\Omega}^{\varepsilon}}^{\star} \boldsymbol{\nu}|D \boldsymbol{u}|^{2} d \boldsymbol{x}+2 \int_{\boldsymbol{\Gamma}}^{\star} \boldsymbol{\gamma}|\boldsymbol{u}|^{2} d \boldsymbol{\sigma}+\gamma_{0} \int_{\boldsymbol{\Gamma}_{0}}^{\star}|\boldsymbol{u}-S \boldsymbol{u}|^{2} d \boldsymbol{\sigma} .
$$

As with $[9,15,25]$, a Korn's inequality is needed for the coercivity of $E(\cdot, \cdot)$.

Lemma 4.1. There is $\varepsilon_{2} \in(0,1]$ such that if $\varepsilon \in\left(0, \varepsilon_{2}\right]$ and $\boldsymbol{u} \in H^{1}\left(\boldsymbol{\Omega}^{\varepsilon}\right)^{3}$ satisfying (2.14) then

$$
\begin{gathered}
|\boldsymbol{\nu}|_{\star}^{1 / 2}\|\boldsymbol{u}\|_{L^{2}} \leq c_{1} \varepsilon^{(1-\delta) / 2} E(\boldsymbol{u})^{1 / 2} \\
|\boldsymbol{\nu}|_{\star}^{1 / 2}\|\boldsymbol{u}\|_{H^{1}} \leq c_{1} E(\boldsymbol{u})^{1 / 2}, \\
E(\boldsymbol{u})^{1 / 2} \leq c_{1}|\boldsymbol{\nu}|_{\star}^{1 / 2}\left(\|\nabla \boldsymbol{u}\|_{L^{2}}+\varepsilon^{(\delta-1) / 2}\|\boldsymbol{u}\|_{L^{2}}\right) \leq \sqrt{2} c_{1} \varepsilon^{(\delta-1) / 2}|\boldsymbol{\nu}|_{\star}^{1 / 2}\|\boldsymbol{u}\|_{H^{1}}
\end{gathered}
$$


where $c_{1}$ is a positive constant independent of $\varepsilon$ and $\boldsymbol{\nu}$.

Proof. By Lemma 4.13 of [9] applied to each domain $\Omega_{\iota}$, we have

$$
\left\|\boldsymbol{\nu}^{1 / 2} \nabla \boldsymbol{u}\right\|_{L^{2}}^{2} \leq 4\left\|\boldsymbol{\nu}^{1 / 2} D \boldsymbol{u}\right\|_{L^{2}}^{2}+C\left\|\boldsymbol{\nu}^{1 / 2} \boldsymbol{u}\right\|_{L^{2}}^{2} \leq 2 E(\boldsymbol{u})+C\left\|\boldsymbol{\nu}^{1 / 2} \boldsymbol{u}\right\|_{L^{2}}^{2} .
$$

By Poincaré's inequality (A.1),

$$
\left\|\boldsymbol{\nu}^{1 / 2} \boldsymbol{u}\right\|_{L^{2}}^{2} \leq C \varepsilon^{2}\left\|\boldsymbol{\nu}^{1 / 2} \nabla \boldsymbol{u}\right\|_{L^{2}}^{2}+C \varepsilon\left\|\boldsymbol{\nu}^{1 / 2} \boldsymbol{u}\right\|_{L^{2}(\boldsymbol{\Gamma})}^{2} .
$$

By relation (2.17), the last $L^{2}$-norm can be bounded by

$$
\left\|\boldsymbol{\nu}^{1 / 2} \boldsymbol{u}\right\|_{L^{2}(\boldsymbol{\Gamma})}^{2} \leq C\left\|\varepsilon^{-\delta / 2} \boldsymbol{\gamma}^{1 / 2} \boldsymbol{u}\right\|_{L^{2}(\boldsymbol{\Gamma})}^{2} \leq C \varepsilon^{-\delta} E(\boldsymbol{u}) .
$$

Hence

$$
\left\|\boldsymbol{\nu}^{1 / 2} \boldsymbol{u}\right\|_{L^{2}}^{2} \leq C \varepsilon^{2}\left\|\boldsymbol{\nu}^{1 / 2} \nabla \boldsymbol{u}\right\|_{L^{2}}^{2}+C \varepsilon^{1-\delta} E(\boldsymbol{u}) .
$$

Using this in (4.10) yields

$$
\left\|\boldsymbol{\nu}^{1 / 2} \nabla \boldsymbol{u}\right\|_{L^{2}}^{2} \leq 2 E(\boldsymbol{u})+C \varepsilon^{2}\left\|\boldsymbol{\nu}^{1 / 2} \nabla \boldsymbol{u}\right\|_{L^{2}}^{2}+C \varepsilon^{1-\delta} E(\boldsymbol{u}) \leq C E(\boldsymbol{u})+C \varepsilon^{2}\left\|\boldsymbol{\nu}^{1 / 2} \nabla \boldsymbol{u}\right\|_{L^{2}}^{2} .
$$

For sufficiently small $\varepsilon$ we obtain

$$
\left\|\boldsymbol{\nu}^{1 / 2} \nabla \boldsymbol{u}\right\|_{L^{2}}^{2} \leq C E(\boldsymbol{u}) .
$$

By (4.11) and (4.12), we obtain (4.7). In turn, (4.7) and (4.12) imply (4.8).

By definition (4.6), property (2.18), relations (2.15) and (2.17) and the trace inequality (A.2),

$$
\begin{aligned}
E(\boldsymbol{u}) & \leq 2|\boldsymbol{\nu}|_{\star}\|\nabla \boldsymbol{u}\|_{L^{2}}^{2}+C \varepsilon^{\delta}|\boldsymbol{\nu}|_{\star}\|\boldsymbol{u}\|_{L^{2}\left(\partial \boldsymbol{\Omega}^{\varepsilon}\right)}^{2} \\
& \leq 2|\boldsymbol{\nu}|_{\star}\|\nabla \boldsymbol{u}\|_{L^{2}}^{2}+C|\boldsymbol{\nu}|_{\star} \varepsilon^{\delta}\left(\varepsilon\|\nabla \boldsymbol{u}\|_{L^{2}}^{2}+\varepsilon^{-1}\|\boldsymbol{u}\|_{L^{2}}^{2}\right) \\
& \leq C|\boldsymbol{\nu}|_{\star}\|\nabla \boldsymbol{u}\|_{L^{2}}^{2}+C|\boldsymbol{\nu}|_{\star} \varepsilon^{\delta-1}\|\boldsymbol{u}\|_{L^{2}}^{2} .
\end{aligned}
$$

Thus one obtains the first inequality in (4.9). The second one is obvious.

We now present the functional formulation for the NSE in the context of boundary conditions (2.14), (2.11) and (2.13). First, we recall the Helmholtz-Leray decomposition:

$$
L^{2}\left(\Omega_{\iota}^{\varepsilon}\right)^{3}=H_{\iota} \oplus H_{\iota}^{\perp},
$$

where

$$
\begin{aligned}
H_{\iota} & =\left\{u \in L^{2}\left(\Omega_{\iota}^{\varepsilon}\right)^{3}: \nabla \cdot u=0 \text { on } \Omega^{\varepsilon}, u \cdot N_{\iota}=0 \text { on } \partial \Omega_{\iota}^{\varepsilon}\right\}, \\
H_{\iota}^{\perp} & =\left\{\nabla \phi: \phi \in H^{1}\left(\Omega_{\iota}^{\varepsilon}\right)\right\} .
\end{aligned}
$$

Then we have for $\Omega^{\varepsilon}$ :

$$
L^{2}\left(\Omega^{\varepsilon}\right)^{3}=H \oplus H^{\perp}
$$

where

$$
\begin{aligned}
H & =H_{+} \oplus H_{-}=\left\{\boldsymbol{u} \in L^{2}\left(\boldsymbol{\Omega}^{\varepsilon}\right)^{3}: \nabla \cdot \boldsymbol{u}=0 \text { on } \boldsymbol{\Omega}^{\varepsilon}, \boldsymbol{u} \cdot \boldsymbol{N}=0 \text { on } \partial \boldsymbol{\Omega}^{\varepsilon}\right\}, \\
H^{\perp} & =H_{+}^{\perp} \oplus H_{-}^{\perp}=\left\{\nabla \boldsymbol{\phi}: \boldsymbol{\phi} \in H^{1}\left(\boldsymbol{\Omega}^{\varepsilon}\right)\right\} .
\end{aligned}
$$


Let $P_{\iota}$ be the orthogonal projection from $L^{2}\left(\Omega_{\iota}^{\varepsilon}\right)^{3}$ onto $H_{\iota}$. Define the Leray-Helmholtz projection for the two-layer domain $\Omega^{\varepsilon}$ by $P=\left[P_{+}, P_{-}\right]=P_{+} \oplus P_{-}$, i.e., $P$ is the orthogonal projection from $L^{2}\left(\Omega^{\varepsilon}\right)^{3}$ onto $H$.

Define $V=H^{1}\left(\Omega^{\varepsilon}\right)^{3} \cap H$.

Consider $\varepsilon \leq \varepsilon_{0}$, where $\varepsilon_{0}$ is from Lemma 4.1. By the same classical arguments as in [4,12] and by Lemma 4.1, there exist a bounded operator $A: V \rightarrow V^{\prime}$ such that

$$
\langle A \boldsymbol{u}, \boldsymbol{v}\rangle_{V^{\prime}, V}=E(\boldsymbol{u}, \boldsymbol{v}), \quad \boldsymbol{u}, \boldsymbol{v} \in V,
$$

and a bi-linear form $B(\cdot, \cdot)$ from $V \times V$ to the dual space $V^{\prime}$, such that

$$
\langle B(\boldsymbol{u}, \boldsymbol{v}), \boldsymbol{w}\rangle_{V^{\prime}, V}=\langle(\boldsymbol{u} \cdot \nabla) \boldsymbol{v}, \boldsymbol{w}\rangle \quad \text { for all } \boldsymbol{u}, \boldsymbol{v}, \boldsymbol{w} \in V .
$$

The domain of $A$, considered as an unbounded operator on $H$, is a subspace $D_{A}$ such that $A \boldsymbol{u} \in H$ for all $\boldsymbol{u} \in D_{A}$. Then $A$ and $D_{A}$ can be characterized by

$$
D_{A}=\left\{\boldsymbol{u} \in H^{2}\left(\boldsymbol{\Omega}^{\varepsilon}\right): \nabla \cdot \boldsymbol{u}=0 \text { and } \boldsymbol{u} \text { satisfies (2.10), (2.11) and (2.12), (2.13) }\right\} \text {. }
$$

and

$$
A \boldsymbol{u}=-\boldsymbol{\nu} P \Delta \boldsymbol{u} \quad \text { for } \boldsymbol{u} \in D_{A} .
$$

We also have $V=D_{A^{1 / 2}}$ - the domain of the fractional power $A^{1 / 2}$.

The Navier-Stokes equations can now be written in the following variational form

$$
\frac{d}{d t}\langle\boldsymbol{u}, \boldsymbol{v}\rangle+E(\boldsymbol{u}, \boldsymbol{v})+\langle B(\boldsymbol{u}, \boldsymbol{u}), \boldsymbol{v}\rangle=\langle\boldsymbol{f}, \boldsymbol{v}\rangle \quad \text { for all } \boldsymbol{v} \in V,
$$

or, equivalently, in the functional form as

$$
\frac{d \boldsymbol{u}}{d t}+A \boldsymbol{u}+B(\boldsymbol{u}, \boldsymbol{u})=P \boldsymbol{f},
$$

in the space $V^{\prime}$. (For more details, see e.g. [5,27].) We have

$$
\begin{gathered}
\langle A \boldsymbol{u}, \boldsymbol{v}\rangle=E(\boldsymbol{u}, \boldsymbol{v}), \quad \boldsymbol{u} \in D_{A}, \boldsymbol{v} \in V, \\
\left\|A^{1 / 2} \boldsymbol{u}\right\|_{L^{2}}^{2}=E(\boldsymbol{u}), \quad \boldsymbol{u} \in V .
\end{gathered}
$$

Below, we find relations between the Lebesgue and Sobolev norms of $\boldsymbol{u}$ with the $A$-related norms $\left\|A^{1 / 2} \boldsymbol{u}\right\|_{L^{2}},\|A \boldsymbol{u}\|_{L^{2}}$.

First, a simple interpolation inequality:

$$
\left\|A^{1 / 2} \boldsymbol{u}\right\|_{L^{2}}^{2}=\langle A \boldsymbol{u}, \boldsymbol{u}\rangle \leq\|\boldsymbol{u}\|_{L^{2}}\|A \boldsymbol{u}\|_{L^{2}}, \quad \boldsymbol{u} \in D_{A} .
$$

Lemma 4.2. There is a positive number $c_{2}$ such that for all $\varepsilon \in\left(0, \varepsilon_{2}\right)$, one has the following:

(i) If $\boldsymbol{u} \in V$ then

$$
\begin{aligned}
& |\boldsymbol{\nu}|_{\star}^{1 / 2}\|\boldsymbol{u}\|_{L^{2}} \leq c_{2} \varepsilon^{(1-\delta) / 2}\left\|A^{1 / 2} \boldsymbol{u}\right\|_{L^{2}}, \\
& |\boldsymbol{\nu}|_{\star}^{1 / 2}\|\boldsymbol{u}\|_{H^{1}} \leq c_{2}\left\|A^{1 / 2} \boldsymbol{u}\right\|_{L^{2}} .
\end{aligned}
$$


(ii) If $\boldsymbol{u} \in D_{A}$ then

$$
\begin{aligned}
|\boldsymbol{\nu}|_{\star}\|\boldsymbol{u}\|_{L^{2}} & \leq c_{2} \varepsilon^{1-\delta}\|A \boldsymbol{u}\|_{L^{2}}, \\
|\boldsymbol{\nu}|_{\star}\|\boldsymbol{u}\|_{H^{1}} & \leq c_{2} \varepsilon^{(1-\delta) / 2}\|A \boldsymbol{u}\|_{L^{2}} \\
|\boldsymbol{\nu}|_{\star}^{1 / 2}\left\|A^{1 / 2} \boldsymbol{u}\right\|_{L^{2}} & \leq c_{2} \varepsilon^{(1-\delta) / 2}\|A \boldsymbol{u}\|_{L^{2}}
\end{aligned}
$$

Proof. Thanks to (4.21), the estimates in (4.23) and (4.24) are restatements of (4.7) and (4.8), respectively. For $\boldsymbol{u} \in D_{A}$ and $u \neq 0$, thanks to (4.22) and (4.23), one has

$$
\left\|A^{1 / 2} \boldsymbol{u}\right\|_{L^{2}}^{2} \leq\|\boldsymbol{u}\|_{L^{2}}\|A \boldsymbol{u}\|_{L^{2}} \leq c_{2} \varepsilon^{(1-\delta) / 2}|\boldsymbol{\nu}|_{\star}^{-1 / 2}\left\|A^{1 / 2} \boldsymbol{u}\right\|_{L^{2}}\|A \boldsymbol{u}\|_{L^{2}},
$$

hence one obtains (4.27). Inequalities (4.24) and (4.27) then imply (4.26). Applying (4.23) and then (4.27), we have (4.25).

Proposition 4.3. If $\boldsymbol{u} \in D_{A}$ then

$$
\|A \boldsymbol{u}+\boldsymbol{\nu} \Delta \boldsymbol{u}\|_{L^{2}} \leq C|\boldsymbol{\nu}|_{\star}\left(\varepsilon^{\delta_{0}}\left\|\nabla^{2} \boldsymbol{u}\right\|_{L^{2}}+\varepsilon^{\delta_{0}-1}\|\nabla \boldsymbol{u}\|_{L^{2}}+\varepsilon^{\delta-1}\|\boldsymbol{u}\|_{L^{2}}\right) .
$$

Proof. Let $\boldsymbol{\omega}=\nabla \times \boldsymbol{u}$ and $\boldsymbol{\Phi}=A \boldsymbol{u}+\boldsymbol{\nu} \Delta \boldsymbol{u}$. Assume $\boldsymbol{\Phi} \neq 0$. By the Helmholtz-Leray decomposition (4.14), $\boldsymbol{\Phi}=\nabla \boldsymbol{q}$ where $\boldsymbol{q}=\left[q_{+}, q_{-}\right] \in H^{1}\left(\boldsymbol{\Omega}^{\varepsilon}\right)$. Since $A \boldsymbol{u} \in H$ and $\boldsymbol{\Phi} \in H^{\perp}$ are orthogonal in $L^{2}\left(\Omega^{\varepsilon}\right)^{3}$, we have

$$
\int_{\boldsymbol{\Omega}^{\varepsilon}}^{\star}|\boldsymbol{\Phi}|^{2} d \boldsymbol{x}=\int_{\boldsymbol{\Omega}^{\varepsilon}}^{\star}(A \boldsymbol{u}+\boldsymbol{\nu} \Delta \boldsymbol{u}) \cdot \boldsymbol{\Phi} d \boldsymbol{x}=\int_{\boldsymbol{\Omega}^{\varepsilon}}^{\star} \boldsymbol{\nu} \Delta \boldsymbol{u} \cdot \boldsymbol{\Phi} d \boldsymbol{x}=-\int_{\boldsymbol{\Omega}^{\varepsilon}}^{\star} \boldsymbol{\nu} \nabla \times \boldsymbol{\omega} \cdot \boldsymbol{\Phi} d \boldsymbol{x} .
$$

By virtue of Lemma 3.8 noting that $\nabla \times \boldsymbol{\Phi}=0$, we obtain

$$
\begin{aligned}
\int_{\boldsymbol{\Omega}^{\varepsilon}}^{\star}|\boldsymbol{\Phi}|^{2} d \boldsymbol{x} & \leq\left|\int_{\boldsymbol{\Omega}^{\varepsilon}}^{\star} \boldsymbol{\nu} \boldsymbol{\Phi} \cdot(\nabla \times G(\boldsymbol{u})) d \boldsymbol{x}\right|+2 \gamma_{0}\left|\int_{\boldsymbol{\Gamma}_{0}}^{\star}[\boldsymbol{N} \times(\boldsymbol{N} \times \boldsymbol{u})] \cdot S \boldsymbol{\Phi} d \boldsymbol{\sigma}\right| \\
& =I_{1}+I_{2} .
\end{aligned}
$$

By Lemma 3.7,

$$
\begin{aligned}
I_{1} & =\left|\int_{\boldsymbol{\Omega}^{\varepsilon}}^{\star} \boldsymbol{\nu} \boldsymbol{\Phi} \cdot(\nabla \times G(\boldsymbol{u})) d \boldsymbol{x}\right| \leq C \int_{\boldsymbol{\Omega}^{\varepsilon}}^{\star} \boldsymbol{\nu}|\boldsymbol{\Phi}|\left(\varepsilon^{\delta}|\nabla \boldsymbol{u}|+\varepsilon^{\delta-1}|\boldsymbol{u}|\right) d \boldsymbol{x} \\
& \leq C|\boldsymbol{\nu}|_{\star}\|\boldsymbol{\Phi}\|_{L^{2}}\left\{\varepsilon^{\delta}\|\nabla \boldsymbol{u}\|_{L^{2}}+\varepsilon^{\delta-1}\|\boldsymbol{u}\|_{L^{2}}\right\} .
\end{aligned}
$$

To estimate $I_{2}$, we use an idea in $[4,15]$. Shifting $q_{\iota}$ by a constant we can assume that $q_{\iota}$ has zero average on $\Omega_{\iota}$. Using a dilation argument (c.f. Lemma 3.8 of [15]), we have

$$
\|\boldsymbol{q}\|_{L^{2}} \leq C\|\nabla \boldsymbol{q}\|_{L^{2}}
$$

Combining this with the trace inequality (A.2) gives

$$
\|\boldsymbol{q}\|_{L^{2}\left(\Gamma_{0}\right)} \leq \frac{C}{\sqrt{\varepsilon}}\|\boldsymbol{q}\|_{L^{2}}+C \sqrt{\varepsilon}\|\nabla \boldsymbol{q}\|_{L^{2}} \leq \frac{C}{\sqrt{\varepsilon}}\|\nabla \boldsymbol{q}\|_{L^{2}}
$$

and hence

$$
\|\boldsymbol{q}\|_{L^{2}\left(\Gamma_{0}\right)} \leq \frac{C}{\sqrt{\varepsilon}}\|\boldsymbol{\Phi}\|_{L^{2}}
$$


Let $\boldsymbol{v}=\boldsymbol{N} \times \boldsymbol{u}$. Then

$$
I_{2}=2 \gamma_{0}|J|, \text { where } J=\int_{\boldsymbol{\Gamma}_{0}}^{\star}[-\boldsymbol{N} \times S \boldsymbol{\Phi}] \cdot \boldsymbol{v} d \boldsymbol{\sigma} .
$$

Noticing $S \boldsymbol{N}=-\boldsymbol{N}$, we have

$$
J=\int_{\boldsymbol{\Gamma}_{0}}^{\star}[S \boldsymbol{N} \times S \boldsymbol{\Phi}] \cdot \boldsymbol{v} d \boldsymbol{\sigma}=\int_{\boldsymbol{\Gamma}_{0}}^{\star}[S(\boldsymbol{N} \times \boldsymbol{\Phi})] \cdot \boldsymbol{v} d \boldsymbol{\sigma}=\int_{\boldsymbol{\Gamma}_{0}}^{\star}(\boldsymbol{N} \times \boldsymbol{\Phi}) \cdot S \boldsymbol{v} d \boldsymbol{\sigma} .
$$

Let $\tilde{\boldsymbol{\tau}}_{1}$ and $\tilde{\boldsymbol{\tau}}_{2}$ be defined in (3.19). We have $\boldsymbol{N}=\tilde{\boldsymbol{\tau}}_{1} \times \tilde{\boldsymbol{\tau}}_{2}$ on $\boldsymbol{\Gamma}_{0}$, thus

$$
\begin{aligned}
J=\int_{\boldsymbol{\Gamma}_{0}}^{\star}(\boldsymbol{N} \times \nabla \boldsymbol{q}) \cdot S \boldsymbol{v} d \boldsymbol{\sigma} & =\int_{\boldsymbol{\Gamma}_{0}}^{\star}\left(\frac{\partial \boldsymbol{q}}{\partial \tilde{\boldsymbol{\tau}}_{1}} \tilde{\boldsymbol{\tau}}_{2}-\frac{\partial \boldsymbol{q}}{\partial \tilde{\boldsymbol{\tau}}_{2}} \tilde{\boldsymbol{\tau}}_{1}\right) \cdot S \boldsymbol{v} d \boldsymbol{\sigma} \\
& =\int_{\boldsymbol{\Gamma}_{0}}^{\star} \frac{\partial \boldsymbol{q}}{\partial \tilde{\boldsymbol{\tau}}_{1}}\left(\tilde{\boldsymbol{\tau}}_{2} \cdot S \boldsymbol{v}\right)-\frac{\partial \boldsymbol{q}}{\partial \tilde{\boldsymbol{\tau}}_{2}}\left(\tilde{\boldsymbol{\tau}}_{1} \cdot S \boldsymbol{v}\right) d \boldsymbol{\sigma} .
\end{aligned}
$$

Applying Lemma 3.5 to this boundary integral on $\Gamma_{0}$ yields

$$
J=\int_{\boldsymbol{\Gamma}_{0}}^{\star}\left(-\boldsymbol{q} \frac{\partial\left(\tilde{\boldsymbol{\tau}}_{2} \cdot S \boldsymbol{v}\right)}{\partial \tilde{\boldsymbol{\tau}}_{1}}+\boldsymbol{q} \frac{\partial\left(\tilde{\boldsymbol{\tau}}_{1} \cdot S \boldsymbol{v}\right)}{\partial \tilde{\boldsymbol{\tau}}_{2}}\right) d \boldsymbol{\sigma}+O(\varepsilon) \int_{\boldsymbol{\Gamma}_{0}}^{\star}\left|\boldsymbol{q}\left(\tilde{\boldsymbol{\tau}}_{1} \cdot S \boldsymbol{v}\right)\right|+\left|\boldsymbol{q}\left(\tilde{\boldsymbol{\tau}}_{2} \cdot S \boldsymbol{v}\right)\right| d \boldsymbol{\sigma} .
$$

Note $|S \boldsymbol{v}|_{\star}=|\boldsymbol{v}|_{\star}=|\boldsymbol{u}|_{\star}$. Then

$$
J=\int_{\Gamma_{0}}^{\star}\left(-\boldsymbol{q} \frac{\partial\left(\tilde{\boldsymbol{\tau}}_{2} \cdot S \boldsymbol{v}\right)}{\partial \tilde{\boldsymbol{\tau}}_{1}}+\boldsymbol{q} \frac{\partial\left(\tilde{\boldsymbol{\tau}}_{1} \cdot S \boldsymbol{v}\right)}{\partial \tilde{\boldsymbol{\tau}}_{2}}\right) d \boldsymbol{\sigma}+O(\varepsilon) \int_{\Gamma_{0}}|\boldsymbol{q}|_{\star}|\boldsymbol{u}|_{\star} d \sigma .
$$

Since $S \tilde{\boldsymbol{\tau}}_{1}=\tilde{\boldsymbol{\tau}}_{1}$ and $S \tilde{\boldsymbol{\tau}}_{2}=-\tilde{\boldsymbol{\tau}}_{2}$, we obtain

$$
\begin{aligned}
J & =\int_{\boldsymbol{\Gamma}_{0}}^{\star}\left(-\boldsymbol{q} \frac{\partial\left(-S \tilde{\boldsymbol{\tau}}_{2} \cdot S \boldsymbol{v}\right)}{\partial\left(S \tilde{\boldsymbol{\tau}}_{1}\right)}+q \frac{\partial\left(S \tilde{\boldsymbol{\tau}}_{1} \cdot S \boldsymbol{v}\right)}{\partial\left(-S \tilde{\boldsymbol{\tau}}_{2}\right)}\right) d \boldsymbol{\sigma}+O(\varepsilon) \int_{\Gamma_{0}}|\boldsymbol{q}|_{\star}|\boldsymbol{u}|_{\star} d \sigma \\
& =\int_{\boldsymbol{\Gamma}_{0}}^{\star}\left(S \boldsymbol{q} \frac{\partial\left(\tilde{\boldsymbol{\tau}}_{2} \cdot \boldsymbol{v}\right)}{\partial \tilde{\boldsymbol{\tau}}_{1}}-S \boldsymbol{q} \frac{\partial\left(\tilde{\boldsymbol{\tau}}_{1} \cdot \boldsymbol{v}\right)}{\partial \tilde{\boldsymbol{\tau}}_{2}}\right) d \boldsymbol{\sigma}+O(\varepsilon) \int_{\Gamma_{0}}|\boldsymbol{q}|_{\star}|\boldsymbol{u}|_{\star} d \sigma .
\end{aligned}
$$

We have

$$
\tilde{\boldsymbol{\tau}}_{1} \cdot \boldsymbol{v}=\boldsymbol{u} \cdot\left(\tilde{\boldsymbol{\tau}}_{1} \times \boldsymbol{N}\right)=-\boldsymbol{u} \cdot \tilde{\boldsymbol{\tau}}_{2} \quad \text { and } \quad \tilde{\boldsymbol{\tau}}_{2} \cdot \boldsymbol{v}=\boldsymbol{u} \cdot\left(\tilde{\boldsymbol{\tau}}_{2} \times \boldsymbol{N}\right)=\boldsymbol{u} \cdot \tilde{\boldsymbol{\tau}}_{1} .
$$

It follows that

$$
\begin{aligned}
J= & \int_{\boldsymbol{\Gamma}_{0}}^{\star} S \boldsymbol{q}\left(\frac{\partial\left(\boldsymbol{u} \cdot \tilde{\boldsymbol{\tau}}_{1}\right)}{\partial \tilde{\boldsymbol{\tau}}_{1}}+\frac{\partial\left(\boldsymbol{u} \cdot \tilde{\boldsymbol{\tau}}_{2}\right)}{\partial \tilde{\boldsymbol{\tau}}_{2}}\right) d \boldsymbol{\sigma}+O(\varepsilon) \int_{\Gamma_{0}}|\boldsymbol{q}|_{\star}|\boldsymbol{u}|_{\star} d \sigma \\
= & \int_{\boldsymbol{\Gamma}_{0}}^{\star} S \boldsymbol{q}\left(\frac{\partial \boldsymbol{u}}{\partial \tilde{\boldsymbol{\tau}}_{1}} \cdot \tilde{\boldsymbol{\tau}}_{1}+\frac{\partial \boldsymbol{u}}{\partial \tilde{\boldsymbol{\tau}}_{2}} \cdot \tilde{\boldsymbol{\tau}}_{2}\right) d \boldsymbol{\sigma}+\int_{\boldsymbol{\Gamma}_{0}}^{\star} S \boldsymbol{q}\left(\boldsymbol{u} \cdot \frac{\partial \tilde{\boldsymbol{\tau}}_{1}}{\partial \tilde{\boldsymbol{\tau}}_{1}}+\boldsymbol{u} \cdot \frac{\partial \tilde{\boldsymbol{\tau}}_{2}}{\partial \tilde{\boldsymbol{\tau}}_{2}}\right) d \boldsymbol{\sigma} \\
& +O(\varepsilon) \int_{\Gamma_{0}}|\boldsymbol{q}|_{\star}|\boldsymbol{u}|_{\star} d \sigma \\
= & \int_{\boldsymbol{\Gamma}_{0}}^{\star} S \boldsymbol{q}\left(\frac{\partial \boldsymbol{u}}{\partial \tilde{\boldsymbol{\tau}}_{1}} \cdot \tilde{\boldsymbol{\tau}}_{1}+\frac{\partial \boldsymbol{u}}{\partial \tilde{\boldsymbol{\tau}}_{2}} \cdot \tilde{\boldsymbol{\tau}}_{2}\right) d \boldsymbol{\sigma}+O(\varepsilon) \int_{\Gamma_{0}}|\boldsymbol{q}|_{\star}|\boldsymbol{u}|_{\star} d \sigma
\end{aligned}
$$

Therefore,

$$
\begin{aligned}
|J| & \leq \int_{\Gamma_{0}}|\boldsymbol{q}|_{\star}|\nabla \boldsymbol{u}|_{\star} d \sigma+O(\varepsilon) \int_{\Gamma_{0}}|\boldsymbol{q}|_{\star}|\boldsymbol{u}|_{\star} d \sigma \\
& \leq C\|\boldsymbol{q}\|_{L^{2}\left(\boldsymbol{\Gamma}_{0}\right)}\left(\|\nabla \boldsymbol{u}\|_{L^{2}\left(\boldsymbol{\Gamma}_{0}\right)}+\varepsilon\|\boldsymbol{u}\|_{L^{2}\left(\boldsymbol{\Gamma}_{0}\right)}\right) .
\end{aligned}
$$


Using (4.30) to estimate $\|\boldsymbol{q}\|_{L^{2}\left(\boldsymbol{\Gamma}_{0}\right)}$ and applying the trace inequality (A.2) to the remaining terms in (4.31), we have

$$
\begin{aligned}
|J| & \leq \frac{C}{\sqrt{\varepsilon}}\|\boldsymbol{\Phi}\|_{L^{2}}\left(\frac{1}{\sqrt{\varepsilon}}\|\nabla \boldsymbol{u}\|_{L^{2}}+\sqrt{\varepsilon}\left\|\nabla^{2} \boldsymbol{u}\right\|_{L^{2}}+\frac{\varepsilon}{\sqrt{\varepsilon}}\|\boldsymbol{u}\|_{L^{2}}+\varepsilon \sqrt{\varepsilon}\|\nabla \boldsymbol{u}\|_{L^{2}}\right) \\
& \leq C\|\boldsymbol{\Phi}\|_{L^{2}}\left(\left\|\nabla^{2} \boldsymbol{u}\right\|_{L^{2}}+\varepsilon^{-1}\|\nabla \boldsymbol{u}\|_{L^{2}}+\varepsilon\|\boldsymbol{u}\|_{L^{2}}\right) .
\end{aligned}
$$

Thus, by (2.17),

$$
I_{2}=2 \gamma_{0}|J| \leq C \varepsilon^{\delta_{0}}|\boldsymbol{\nu}|_{\star}\|\boldsymbol{\Phi}\|_{L^{2}}\left(\left\|\nabla^{2} \boldsymbol{u}\right\|_{L^{2}}+\varepsilon^{-1}\|\nabla \boldsymbol{u}\|_{L^{2}}+\varepsilon\|\boldsymbol{u}\|_{L^{2}}\right) .
$$

Combining above estimates for $I_{1}$ and $I_{2}$ with (4.29) gives

$$
\begin{aligned}
\|\boldsymbol{\Phi}\|_{L^{2}}^{2} \leq I_{1}+I_{2} \leq C|\boldsymbol{\nu}|_{\star} & \|\boldsymbol{\Phi}\|_{L^{2}}\left\{\varepsilon^{\delta}\|\nabla \boldsymbol{u}\|_{L^{2}}+\varepsilon^{\delta-1}\|\boldsymbol{u}\|_{L^{2}}\right\} \\
& +C|\boldsymbol{\nu}|_{\star}\|\boldsymbol{\Phi}\|_{L^{2}}\left(\varepsilon^{\delta_{0}}\left\|\nabla^{2} \boldsymbol{u}\right\|_{L^{2}}+\varepsilon^{\delta_{0}-1}\|\nabla \boldsymbol{u}\|_{L^{2}}+\varepsilon^{\delta_{0}+1}\|\boldsymbol{u}\|_{L^{2}}\right) .
\end{aligned}
$$

Then dividing both sides of this inequality by $\|\Phi\|_{L^{2}} \neq 0$ yields

$$
\|A \boldsymbol{u}+\boldsymbol{\nu} \Delta \boldsymbol{u}\|_{L^{2}}=\|\boldsymbol{\Phi}\|_{L^{2}} \leq C|\boldsymbol{\nu}|_{\star}\left(\varepsilon^{\delta_{0}}\left\|\nabla^{2} \boldsymbol{u}\right\|_{L^{2}}+\varepsilon^{\delta_{0}-1}\|\nabla \boldsymbol{u}\|_{L^{2}}+\varepsilon^{\delta-1}\|\boldsymbol{u}\|_{L^{2}}\right),
$$

hence we obtain (4.28).

Proposition 4.3 above is a counterpart of Corollary 5.3 in [8] and Theorem 2.1 in [7]. However, its proof is more involved due to the interface boundary condition expressed in $I_{2}$, which results in the term $\left\|\nabla^{2} \boldsymbol{u}\right\|_{L^{2}}$ of higher derivatives. (See also $[4,15]$ for similar estimates.)

The equivalence between two norms $\|A u\|_{L^{2}}$ and $\|u\|_{H^{2}}$ then follows.

Proposition 4.4. There is $0<\varepsilon_{*} \leq \min \left\{\varepsilon_{1}, \varepsilon_{2}\right\}$ such that if $\varepsilon \in\left(0, \varepsilon_{*}\right]$ and $\boldsymbol{u} \in D_{A}$, then

$$
C^{-1}\|A \boldsymbol{u}\|_{L^{2}} \leq|\boldsymbol{\nu}|_{\star}\|\boldsymbol{u}\|_{H^{2}} \leq C\left(1+\varepsilon^{\delta_{0}-(1+\delta) / 2}\right)\|A \boldsymbol{u}\|_{L^{2}}
$$

Proof. Let $0<\varepsilon \leq \min \left\{\varepsilon_{1}, \varepsilon_{2}\right\}$ and $\boldsymbol{u} \in D_{A}$. On one hand,

$$
\|A \boldsymbol{u}\|_{L^{2}}=\|\boldsymbol{\nu} P(-\Delta \boldsymbol{u})\|_{L^{2}} \leq|\boldsymbol{\nu}|_{\star}\|\Delta \boldsymbol{u}\|_{L^{2}} \leq C|\boldsymbol{\nu}|_{\star}\|\boldsymbol{u}\|_{H^{2}} .
$$

On the other hand, it follows from Lemma 3.6 and Proposition 4.3 that

$$
\begin{aligned}
\|\boldsymbol{u}\|_{H^{2}} & \leq C\|\boldsymbol{u}\|_{H^{1}}+\left\|\nabla^{2} \boldsymbol{u}\right\|_{L^{2}} \leq C\|\boldsymbol{u}\|_{H^{1}}+C\left(\|\Delta \boldsymbol{u}\|_{L^{2}}+C\|\boldsymbol{u}\|_{H^{1}}\right) \\
& \leq C\|\boldsymbol{u}\|_{H^{1}}+C\left(\left\|\Delta \boldsymbol{u}+\nu^{-1} A \boldsymbol{u}\right\|_{L^{2}}+\left\|-\nu^{-1} A \boldsymbol{u}\right\|_{L^{2}}\right) \\
& \leq C\|\boldsymbol{u}\|_{H^{1}}+C\left(\varepsilon^{\delta_{0}}\left\|\nabla^{2} \boldsymbol{u}\right\|_{L^{2}}+\varepsilon^{\delta_{0}-1}\|\nabla \boldsymbol{u}\|_{L^{2}}+\varepsilon^{\delta-1}\|\boldsymbol{u}\|_{L^{2}}\right)+C|\boldsymbol{\nu}|_{\star}^{-1}\|A \boldsymbol{u}\|_{L^{2}} \\
& \leq C \varepsilon^{\delta_{0}}\|\boldsymbol{u}\|_{H^{2}}+C|\boldsymbol{\nu}|_{\star}^{-1}\|A \boldsymbol{u}\|_{L^{2}}+C \varepsilon^{\delta_{0}-1}\|\boldsymbol{u}\|_{H^{1}}+C \varepsilon^{\delta-1}\|\boldsymbol{u}\|_{L^{2}} .
\end{aligned}
$$

Since $\delta_{0}>0$, for $\varepsilon$ sufficiently small, absorbing $C \varepsilon^{\delta_{0}}\|\boldsymbol{u}\|_{H^{2}}$ into the left-hand side, using (4.25) and (4.27) we obtain

$$
\begin{aligned}
\|\boldsymbol{u}\|_{H^{2}} & \leq C|\boldsymbol{\nu}|_{\star}^{-1}\|A \boldsymbol{u}\|_{L^{2}}+C \varepsilon^{\delta_{0}-1}\left(\varepsilon^{(1-\delta) / 2}|\boldsymbol{\nu}|_{\star}^{-1}\left\|A^{1 / 2} \boldsymbol{u}\right\|_{L^{2}}\right)+C \varepsilon^{\delta-1}\left(\varepsilon^{1-\delta}|\boldsymbol{\nu}|_{\star}^{-1}\|A \boldsymbol{u}\|_{L^{2}}\right) \\
& \leq C\left(1+\varepsilon^{\delta_{0}-(1+\delta) / 2}\right)|\boldsymbol{\nu}|_{\star}^{-1}\|A \boldsymbol{u}\|_{L^{2}} .
\end{aligned}
$$

Thus the second inequality in (4.32) follows. 
Corollary 4.5. If $\delta_{0}=1, \varepsilon \in\left(0, \varepsilon_{*}\right]$ and $\boldsymbol{u} \in D_{A}$ then

$$
C^{-1}\|A \boldsymbol{u}\|_{L^{2}} \leq|\boldsymbol{\nu}|_{\star}\|\boldsymbol{u}\|_{H^{2}} \leq C\|A \boldsymbol{u}\|_{L^{2}}
$$

and

$$
\|A \boldsymbol{u}+\boldsymbol{\nu} \Delta \boldsymbol{u}\|_{L^{2}} \leq C\left(\varepsilon\|A \boldsymbol{u}\|_{L^{2}}+|\boldsymbol{\nu}|_{\star}^{1 / 2}\left\|A^{1 / 2} \boldsymbol{u}\right\|_{L^{2}}+|\boldsymbol{\nu}|_{\star} \varepsilon^{\delta-1}\|\boldsymbol{u}\|_{L^{2}}\right) .
$$

Proof. In this case $\delta_{0}-(1+\delta) / 2=(1-\delta) / 2 \geq 0$, hence (4.33) follows (4.32).

By (4.28),

$$
\|A \boldsymbol{u}+\boldsymbol{\nu} \Delta \boldsymbol{u}\|_{L^{2}} \leq C|\boldsymbol{\nu}|_{\star}\left(\varepsilon\left\|\nabla^{2} \boldsymbol{u}\right\|_{L^{2}}+\|\nabla \boldsymbol{u}\|_{L^{2}}+\varepsilon^{\delta-1}\|\boldsymbol{u}\|_{L^{2}}\right) .
$$

Applying (4.33) and (4.24) to estimate the first two norms on the right-hand side, we obtain (4.34).

4.2. Averaging operators. We extend the averaging operators $M_{0}$ and $M$ in [8] (see also [9, 15]) for single layer domains to the two-layer ones. Their properties are stated below (c.f. [9] for the proofs).

First, we define an averaging operator $M_{0}$ on $L^{2}\left(\Omega^{\varepsilon}\right)$ by $M_{0}=\left[M_{0}^{+}, M_{0}^{-}\right]$where

$$
\left(M_{0}^{\iota} \phi\right)(x)=\frac{1}{\varepsilon \iota g_{\iota}\left(x^{\prime}\right)} \int_{h_{0}\left(x^{\prime}\right)}^{h_{\iota}\left(x^{\prime}\right)} \phi\left(x^{\prime}, y_{3}\right) d y_{3}, \quad x=\left(x^{\prime}, x_{3}\right) \in \mathbb{T}^{2} \times \mathbb{R},
$$

for $\iota=+,-$ and $\phi \in L^{2}\left(\Omega_{\iota}^{\varepsilon}\right)$. Then

$$
M_{0} \phi=\left[M_{0}^{+} \phi_{+}, M_{0}^{-} \phi_{-}\right] \text {for } \phi=\left[\phi_{+}, \phi_{-}\right] \in L^{2}\left(\Omega^{\varepsilon}\right)^{3} .
$$

Note that $\left(M_{0} \phi\right)(x)$ is independent of $x_{3}$. Also, $M_{0}$ is an orthogonal projection on $L^{2}\left(\Omega^{\varepsilon}\right)$, hence

$$
\left\|M_{0} \phi\right\|_{L^{2}}^{2}+\left\|\phi-M_{0} \phi\right\|_{L^{2}}^{2}=\|\phi\|_{L^{2}}^{2} .
$$

Define a function $\boldsymbol{\psi}=\left[\psi_{+}, \psi_{-}\right]=\left(\boldsymbol{\psi}_{1}, \boldsymbol{\psi}_{2}\right)$ on $\mathbb{T}^{2} \times \mathbb{R}$ by

$$
\psi_{\iota}(x)=\left(\psi_{\iota, 1}, \psi_{\iota, 2}\right)(x) \stackrel{\text { def }}{=} \frac{1}{\varepsilon \iota g_{\iota}\left(x^{\prime}\right)}\left\{\left(x_{3}-h_{0}\right) \nabla_{2} h_{\iota}\left(x^{\prime}\right)+\left(h_{\iota}\left(x^{\prime}\right)-x_{3}\right) \nabla_{2} h_{0}\left(x^{\prime}\right)\right\}
$$

for $\iota=+,-$, and $x=\left(x^{\prime}, x_{3}\right)=\left(x_{1}, x_{2}, x_{3}\right) \in \mathbb{T}^{2} \times \mathbb{R}$, where we recall $\nabla_{2}=\left(\partial / \partial x_{1}, \partial / \partial x_{2}\right)$.

Note that

$$
\begin{gathered}
\left.\psi_{\iota}\right|_{\Gamma_{0}}=\nabla_{2} h_{0},\left.\quad \psi_{\iota}\right|_{\Gamma_{\iota}}=\nabla_{2} h_{\iota}, \quad \partial_{3} \psi_{\iota}=\left(1 / g_{\iota}\right) \nabla_{2} g_{\iota}, \quad \iota=+,-, \\
|\boldsymbol{\psi}| \leq C \varepsilon, \quad\left|\partial_{3} \boldsymbol{\psi}\right| \leq C, \quad\left|\nabla_{2} \boldsymbol{\psi}\right| \leq C \varepsilon, \quad\left|\nabla^{2} \boldsymbol{\psi}\right| \leq C .
\end{gathered}
$$

Let $\phi \in H^{1}\left(\Omega^{\varepsilon}\right)$ and $j=1,2$, then

$$
\partial_{j} M_{0} \boldsymbol{\phi}=M_{0}\left(\partial_{j} \phi\right)+M_{0}\left(\boldsymbol{\psi}_{j} \partial_{3} \boldsymbol{\phi}\right) .
$$

Consequently, for $m=0,1,2$, and $\phi \in H^{m}\left(\boldsymbol{\Omega}^{\varepsilon}\right)$,

$$
\left\|M_{0} \boldsymbol{\phi}\right\|_{H^{m}\left(\boldsymbol{\Omega}^{\varepsilon}\right)} \leq C(m)\|\boldsymbol{\phi}\|_{H^{m}\left(\boldsymbol{\Omega}^{\varepsilon}\right)},
$$

where positive number $C(m)$ is independent of $\varepsilon$. 
Let $\boldsymbol{u} \in H$ and $\overline{\boldsymbol{v}}=\left(M_{0} \boldsymbol{u}_{1}, M_{0} \boldsymbol{u}_{2}\right)$. Then

$$
\nabla_{2} \cdot \overline{\boldsymbol{v}}=-\overline{\boldsymbol{v}} \cdot \frac{1}{\boldsymbol{g}} \nabla_{2} \boldsymbol{g}, \quad \text { where } \boldsymbol{g}=\left[g_{+}, g_{-}\right] .
$$

Next, we define the operator $M$. For $\boldsymbol{u}=\left(\boldsymbol{u}_{1}, \boldsymbol{u}_{2}, \boldsymbol{u}_{3}\right) \in L^{2}\left(\Omega^{\varepsilon}\right)^{3}$, let

$$
\overline{\boldsymbol{v}}=\left(M_{0} \boldsymbol{u}_{1}, M_{0} \boldsymbol{u}_{2}\right)
$$

and define

$$
M \boldsymbol{u}=(\overline{\boldsymbol{v}}, \overline{\boldsymbol{v}} \cdot \boldsymbol{\psi})=\left(M_{0} \boldsymbol{u}_{1}, M_{0} \boldsymbol{u}_{2}, \boldsymbol{\psi}_{1} M_{0} \boldsymbol{u}_{1}+\boldsymbol{\psi}_{2} M_{0} \boldsymbol{u}_{2}\right),
$$

Same as in [9] we have that $M \boldsymbol{u} \in H$ provided $\boldsymbol{u} \in H$.

Let $\boldsymbol{u} \in H^{1}\left(\boldsymbol{\Omega}^{\varepsilon}\right)^{3}$ and $\boldsymbol{v}=M \boldsymbol{u}=\left(\overline{\boldsymbol{v}}, \boldsymbol{v}_{3}\right)$. Then we have

$$
\left|\boldsymbol{v}_{3}\right| \leq C \varepsilon|\overline{\boldsymbol{v}}|, \quad\left|\partial_{3} \boldsymbol{v}_{3}\right| \leq C|\overline{\boldsymbol{v}}|, \quad\left|\nabla_{2} \boldsymbol{v}_{3}\right| \leq C \varepsilon\left(|\overline{\boldsymbol{v}}|+\left|\nabla_{2} \overline{\boldsymbol{v}}\right|\right) \quad \text { in } \Omega^{\varepsilon}
$$

and, consequently,

$$
|\boldsymbol{v}| \leq C|\overline{\boldsymbol{v}}|, \quad|\nabla \boldsymbol{v}| \leq C\left(|\overline{\boldsymbol{v}}|+\left|\nabla_{2} \overline{\boldsymbol{v}}\right|\right) \quad \text { in } \Omega^{\varepsilon} .
$$

Combining (4.45) with (4.40) and (4.41), we have for $m=0,1,2$, and $\boldsymbol{u} \in H^{m}\left(\boldsymbol{\Omega}^{\varepsilon}\right)$ that

$$
\|\boldsymbol{v}\|_{H^{m}\left(\boldsymbol{\Omega}^{\varepsilon}\right)} \leq C(m)\|\boldsymbol{u}\|_{H^{m}\left(\boldsymbol{\Omega}^{\varepsilon}\right)} .
$$

Applying 2D Ladyzhenskaya inequality (A.6) to $\overline{\boldsymbol{v}}$, we derive from (4.45) that

$$
\begin{aligned}
\|\boldsymbol{v}\|_{L^{4}} \leq C \varepsilon^{-1 / 4}\|\boldsymbol{u}\|_{L^{2}}^{1 / 2}\|\boldsymbol{u}\|_{H^{1}}^{1 / 2} & \text { for } \boldsymbol{u} \in H^{1}\left(\boldsymbol{\Omega}^{\varepsilon}\right)^{3}, \\
\|\nabla \boldsymbol{v}\|_{L^{4}} \leq C \varepsilon^{-1 / 4}\|\boldsymbol{u}\|_{H^{1}}^{1 / 2}\|\boldsymbol{u}\|_{H^{2}}^{1 / 2} & \text { for } \boldsymbol{u} \in H^{2}\left(\Omega^{\varepsilon}\right)^{3} .
\end{aligned}
$$

Now, let $\boldsymbol{w}=\boldsymbol{u}-M \boldsymbol{u}$. The following Poincaré's inequality for $\boldsymbol{w}$ is from Lemma 3.5 of [9]:

Lemma 4.6. If $\varepsilon \in\left(0, \varepsilon_{*}\right]$ and $\boldsymbol{u} \in H^{1}\left(\Omega^{\varepsilon}\right)^{3}$ satisfies the boundary condition (2.14) then

$$
\|\boldsymbol{w}\|_{L^{2}} \leq C \varepsilon\|\nabla \boldsymbol{w}\|_{L^{2}}
$$

Consequently,

$$
\|\boldsymbol{w}\|_{L^{2}} \leq C \varepsilon|\boldsymbol{\nu}|_{\star}^{-1 / 2}\left\|A^{1 / 2} \boldsymbol{u}\right\|_{L^{2}}, \quad \boldsymbol{u} \in V .
$$

Above, inequality (4.50) results from (4.49) and (4.24).

Lemma 4.7. Assume $\delta_{0}=1$ and $\varepsilon \in\left(0, \varepsilon_{*}\right]$.

(i) Poincaré inequalities. If $\boldsymbol{u} \in D_{A}$ then

$$
\begin{gathered}
\|\nabla \boldsymbol{w}\|_{L^{2}} \leq C \varepsilon|\boldsymbol{\nu}|_{\star}^{-1}\|A \boldsymbol{u}\|_{L^{2}} \leq C \varepsilon\|\boldsymbol{u}\|_{H^{2}}, \\
\|\boldsymbol{w}\|_{L^{2}} \leq C \varepsilon^{2}|\boldsymbol{\nu}|_{\star}^{-1}\|A \boldsymbol{u}\|_{L^{2}} \leq C \varepsilon^{2}\|\boldsymbol{u}\|_{H^{2}}
\end{gathered}
$$

and, consequently,

$$
\|\boldsymbol{w}\|_{H^{1}} \leq C \varepsilon|\boldsymbol{\nu}|_{\star}^{-1}\|A \boldsymbol{u}\|_{L^{2}} \leq C \varepsilon\|\boldsymbol{u}\|_{H^{2}}
$$


(ii) Sobolev inequalities. If $\boldsymbol{u} \in V$ then

$$
\|\boldsymbol{w}\|_{L^{6}} \leq C\|\boldsymbol{w}\|_{H^{1}} \leq C|\boldsymbol{\nu}|_{\star}^{-1}\left\|A^{1 / 2} \boldsymbol{u}\right\|_{L^{2}} .
$$

If $\boldsymbol{u} \in D_{A}$ then

$$
\|\nabla \boldsymbol{w}\|_{L^{6}} \leq C|\boldsymbol{\nu}|_{\star}^{-1}\|A \boldsymbol{u}\|_{L^{2}} \leq C\|\boldsymbol{u}\|_{H^{2}}
$$

(iii) Agmon inequality. If $\boldsymbol{u} \in D_{A}$ then

$$
\|\boldsymbol{w}\|_{L^{\infty}} \leq C \varepsilon^{1 / 2}|\boldsymbol{\nu}|_{\star}^{-1}\|A \boldsymbol{u}\|_{L^{2}} .
$$

Proof. (i) Same as Lemma 4.3 in [8],

$$
\left\|\partial_{i} \boldsymbol{w}_{j}\right\|_{L^{2}},\left\|\partial_{3} \boldsymbol{w}_{3}\right\|_{L^{2}},\left\|\partial_{i} \boldsymbol{w}_{3}\right\|_{L^{2}} \leq C \varepsilon\|\boldsymbol{u}\|_{H^{2}}, \quad i, j=1,2 .
$$

By Lemma 3.4, (2.16) and (2.17),

$$
\begin{aligned}
\left\|\partial_{3} \boldsymbol{w}_{j}\right\|_{L^{2}}=\left\|\partial_{3} \boldsymbol{u}_{j}\right\|_{L^{2}} & \leq C\left(\varepsilon\|\boldsymbol{u}\|_{H^{2}}+\varepsilon^{1 / 2} \frac{1}{|\boldsymbol{\nu}|_{\star}^{1 / 2}}\left\|\left(\frac{\gamma}{\boldsymbol{\nu}}\right)^{1 / 2} \cdot \boldsymbol{\gamma}^{1 / 2} \boldsymbol{u}\right\|_{L^{2}(\boldsymbol{\Gamma})}\right) \\
& \leq C\left(\varepsilon\|\boldsymbol{u}\|_{H^{2}}+\varepsilon^{1 / 2} \varepsilon^{\delta / 2}|\boldsymbol{\nu}|_{\star}^{-1 / 2}\left\|A^{1 / 2} \boldsymbol{u}\right\|_{L^{2}}\right) .
\end{aligned}
$$

Summing up the above estimates for $\left\|\partial_{i} \boldsymbol{w}_{j}\right\|_{L^{2}}$, for $i, j=1,2,3$, and using (4.33) and (4.27), we obtain

$$
\begin{aligned}
\|\nabla \boldsymbol{w}\|_{L^{2}} & \leq C \varepsilon|\boldsymbol{\nu}|_{\star}^{-1}\|A \boldsymbol{u}\|_{L^{2}}+C \varepsilon^{(1+\delta) / 2}|\boldsymbol{\nu}|_{\star}^{-1 / 2}\left(\varepsilon^{(1-\delta) / 2}|\boldsymbol{\nu}|_{\star}^{-1 / 2}\|A \boldsymbol{u}\|_{L^{2}}\right) \\
& \leq C \varepsilon|\boldsymbol{\nu}|_{\star}^{-1}\|A \boldsymbol{u}\|_{L^{2}},
\end{aligned}
$$

hence inequality (4.51) follows. This and (4.49) imply (4.52).

(ii) and (iii) Now that we have (i), the proofs of (ii) and (iii) can be proceeded the same way as in Lemmas 4.4 and 4.5 of [8].

\section{ESTIMATE OF A NONLINEAR TERM}

Regarding the nonlinear term in the NSE, here is the main estimate of the paper.

Proposition 5.1. Assume

$$
2 / 3 \leq \delta \leq 1 \text { and } \delta_{0}=1
$$

Given $\alpha>0$, there is $C_{\alpha}>0$ such that for any $\varepsilon \in\left(0, \varepsilon_{*}\right]$ and $\boldsymbol{u} \in D_{A}$, we have

$$
\begin{aligned}
|\langle B(\boldsymbol{u}, \boldsymbol{u}), A \boldsymbol{u}\rangle|= & \left|\int_{\boldsymbol{\Omega}^{\varepsilon}}^{\star}[(\boldsymbol{u} \cdot \nabla) \boldsymbol{u}] \cdot A \boldsymbol{u} d \boldsymbol{x}\right| \\
\leq & \alpha\|A \boldsymbol{u}\|_{L^{2}}^{2}+c_{3} \varepsilon^{1 / 2}|\boldsymbol{\nu}|_{\star}^{-3 / 2}\left\|A^{1 / 2} \boldsymbol{u}\right\|_{L^{2}}\|A \boldsymbol{u}\|_{L^{2}}^{2} \\
& +C_{\alpha} \varepsilon^{-1}\left(|\boldsymbol{\nu}|_{\star}^{-1}\|\boldsymbol{u}\|_{L^{2}}^{2}+\|\boldsymbol{u}\|_{L^{2}}\right)\left\|A^{1 / 2} \boldsymbol{u}\right\|_{L^{2}}^{2} .
\end{aligned}
$$

where $\varepsilon_{*}$ is from Proposition 4.4, and $c_{3}>0$ is independent of $\varepsilon, \boldsymbol{\nu}, \alpha$ and $\boldsymbol{u}$. 
Proof. The equality in (5.2) comes from the fact that $B(\boldsymbol{u}, \boldsymbol{u})=P[(\boldsymbol{u} \cdot \nabla) \boldsymbol{u}]$ and $P A \boldsymbol{u}=A \boldsymbol{u}$.

We now prove the inequality. The proof follows the key steps in Proposition 6.1 of [8] and uses many calculations there. Let $\boldsymbol{v}=M \boldsymbol{u}$ and $\boldsymbol{w}=\boldsymbol{u}-\boldsymbol{v}$, also $\boldsymbol{\omega}=\nabla \times \boldsymbol{u}$ and $\boldsymbol{\Phi}=\boldsymbol{v} \times \boldsymbol{\omega}$. We first rewrite

$$
\begin{aligned}
I & \stackrel{\text { def }}{=}-\int_{\boldsymbol{\Omega}^{\varepsilon}}^{\star}(\boldsymbol{u} \cdot \nabla) \boldsymbol{u} \cdot A \boldsymbol{u} d \boldsymbol{x}=\int_{\boldsymbol{\Omega}^{\varepsilon}}^{\star}\left\{\boldsymbol{u} \times(\nabla \times \boldsymbol{u})-\frac{1}{2} \nabla|\boldsymbol{u}|^{2}\right\} \cdot A \boldsymbol{u} d \boldsymbol{x} \\
& =\int_{\boldsymbol{\Omega}^{\varepsilon}}^{\star}(\boldsymbol{u} \times \boldsymbol{\omega}) \cdot A \boldsymbol{u} d \boldsymbol{x}=\int_{\boldsymbol{\Omega}^{\varepsilon}}^{\star}(\boldsymbol{w} \times \boldsymbol{\omega}) \cdot A \boldsymbol{u} d \boldsymbol{x}+\int_{\boldsymbol{\Omega}^{\varepsilon}}^{\star}(\boldsymbol{v} \times \boldsymbol{\omega}) \cdot A \boldsymbol{u} d \boldsymbol{x} \\
& =J_{1}+J_{2}-J_{3}
\end{aligned}
$$

where

$$
J_{1}=\int_{\boldsymbol{\Omega}^{\varepsilon}}^{\star}(\boldsymbol{w} \times \boldsymbol{\omega}) \cdot A \boldsymbol{u} d \boldsymbol{x}, J_{2}=\int_{\boldsymbol{\Omega}^{\varepsilon}}^{\star} \boldsymbol{\Phi} \cdot(A \boldsymbol{u}+\boldsymbol{\nu} \Delta \boldsymbol{u}) d \boldsymbol{x}, J_{3}=\int_{\boldsymbol{\Omega}^{\varepsilon}}^{\star} \boldsymbol{\Phi} \cdot \boldsymbol{\nu} \Delta \boldsymbol{u} d \boldsymbol{x} .
$$

Estimate of $J_{1}$. By Agmon's inequality (4.56), Hölder's inequality and relation (4.24)

$$
\begin{aligned}
\left|J_{1}\right| & \leq\|\boldsymbol{w}\|_{L^{\infty}}\|\boldsymbol{\omega}\|_{L^{2}}\|A \boldsymbol{u}\|_{L^{2}} \\
& \leq C\left(\varepsilon^{1 / 2}|\boldsymbol{\nu}|_{\star}^{-1}\|A \boldsymbol{u}\|_{L^{2}}\right)\left(|\boldsymbol{\nu}|_{\star}^{-1 / 2}\left\|A^{1 / 2} \boldsymbol{u}\right\|_{L^{2}}\right)\|A \boldsymbol{u}\|_{L^{2}}
\end{aligned}
$$

hence

$$
\left|J_{1}\right| \leq C \varepsilon^{1 / 2}|\boldsymbol{\nu}|_{\star}^{-3 / 2}\left\|A^{1 / 2} \boldsymbol{u}\right\|_{L^{2}}\|A \boldsymbol{u}\|_{L^{2}}^{2} .
$$

Estimate of $J_{2}$. First, we apply (4.45) and Cao-Titi inequality (A.8) to have

$$
\begin{aligned}
\|\boldsymbol{\Phi}\|_{L^{2}} & \leq C \varepsilon^{-1 / 2}\|\overline{\boldsymbol{v}}\|_{L^{2}}^{1 / 2}\|\overline{\boldsymbol{v}}\|_{H^{1}}^{1 / 2}\|\boldsymbol{\omega}\|_{L^{2}}^{1 / 2}\|\boldsymbol{\omega}\|_{H^{1}}^{1 / 2} \\
& \leq C \varepsilon^{-1 / 2}\|\boldsymbol{u}\|_{L^{2}}^{1 / 2}\|\boldsymbol{u}\|_{H^{1}}\|\boldsymbol{u}\|_{H^{2}}^{1 / 2}
\end{aligned}
$$

hence by Lemma 4.2 and Corollary 4.5 ,

$$
\|\boldsymbol{\Phi}\|_{L^{2}} \leq C \varepsilon^{-1 / 2}|\boldsymbol{\nu}|_{\star}^{-1}\|\boldsymbol{u}\|_{L^{2}}^{1 / 2}\left\|A^{1 / 2} \boldsymbol{u}\right\|_{L^{2}}\|A \boldsymbol{u}\|_{L^{2}}^{1 / 2} .
$$

By Hölder's inequality and (4.34),

$$
\begin{aligned}
\left|J_{2}\right| & \leq\|\boldsymbol{\Phi}\|_{L^{2}}\|A \boldsymbol{u}+\boldsymbol{\nu} \Delta \boldsymbol{u}\|_{L^{2}} \\
& \leq C\|\boldsymbol{\Phi}\|_{L^{2}}\left(\varepsilon\|A \boldsymbol{u}\|_{L^{2}}+C|\boldsymbol{\nu}|_{\star}^{1 / 2}\left\|A^{1 / 2} \boldsymbol{u}\right\|_{L^{2}}+\varepsilon^{\delta-1}|\boldsymbol{\nu}|_{\star}\|\boldsymbol{u}\|_{L^{2}}\right) .
\end{aligned}
$$

Using (5.4), we have

$$
\left|J_{2}\right| \leq C\left(J_{2,1}+J_{2,2}+J_{2,3}\right)
$$

where

$$
\begin{aligned}
& J_{2,1}=\varepsilon^{1 / 2}|\boldsymbol{\nu}|_{\star}^{-1}\|\boldsymbol{u}\|_{L^{2}}^{1 / 2}\left\|A^{1 / 2} \boldsymbol{u}\right\|_{L^{2}}\|A \boldsymbol{u}\|_{L^{2}}^{3 / 2}, \\
& J_{2,2}=\varepsilon^{-1 / 2}|\boldsymbol{\nu}|_{\star}^{-1 / 2}\|\boldsymbol{u}\|_{L^{2}}^{1 / 2}\left\|A^{1 / 2} \boldsymbol{u}\right\|_{L^{2}}^{2}\|A \boldsymbol{u}\|_{L^{2}}^{1 / 2}, \\
& J_{2,3}=\varepsilon^{\delta-3 / 2}\|\boldsymbol{u}\|_{L^{2}}^{3 / 2}\left\|A^{1 / 2} \boldsymbol{u}\right\|_{L^{2}}\|A \boldsymbol{u}\|_{L^{2}}^{1 / 2} .
\end{aligned}
$$


Using (4.25) to estimate $\|\boldsymbol{u}\|_{L^{2}}$ in $J_{2,1}$ gives

$$
J_{2,1} \leq C \varepsilon^{1 / 2}|\boldsymbol{\nu}|_{\star}^{-3 / 2}\left\|A^{1 / 2} \boldsymbol{u}\right\|_{L^{2}}\|A \boldsymbol{u}\|_{L^{2}}^{2} .
$$

We define $J_{0}=\varepsilon^{-1 / 2}|\boldsymbol{\nu}|_{\star}^{-1 / 2}\|\boldsymbol{u}\|_{L^{2}}\left\|A^{1 / 2} \boldsymbol{u}\right\|_{L^{2}}\|A \boldsymbol{u}\|_{L^{2}}$ and use it as an intermediate term aiding other estimates. By Young's inequality,

$$
J_{0} \leq \alpha^{\prime}\|A \boldsymbol{u}\|_{L^{2}}^{2}+C_{\alpha^{\prime}} \varepsilon^{-1}|\boldsymbol{\nu}|_{\star}^{-1}\|\boldsymbol{u}\|_{L^{2}}^{2}\left\|A^{1 / 2} \boldsymbol{u}\right\|_{L^{2}}^{2} \quad \text { for all } \alpha^{\prime}>0 .
$$

Using interpolation inequality (4.22) to estimate $\left\|A^{1 / 2} \boldsymbol{u}\right\|_{L^{2}}$, but not $\left\|A^{1 / 2} \boldsymbol{u}\right\|_{L^{2}}^{2}$, in $J_{2,2}$ and then applying (5.5), we have

$$
J_{2,2} \leq J_{0} \leq \alpha\|A \boldsymbol{u}\|_{L^{2}}^{2}+C_{\alpha} \varepsilon^{-1}|\boldsymbol{\nu}|_{\star}^{-1}\|\boldsymbol{u}\|_{L^{2}}^{2}\left\|A^{1 / 2} \boldsymbol{u}\right\|_{L^{2}}^{2} .
$$

For $J_{2,3}$, applying Young's inequality yields

$$
J_{2,3} \leq \alpha\|A \boldsymbol{u}\|_{L^{2}}^{2}+C_{\alpha} \varepsilon^{4 \delta / 3-2}\|\boldsymbol{u}\|_{L^{2}}^{2}\left\|A^{1 / 2} \boldsymbol{u}\right\|_{L^{2}}^{4 / 3} .
$$

Using (4.23) to estimate $\|\boldsymbol{u}\|_{L^{2}}^{2 / 3}$ in the last product gives

$$
\begin{aligned}
J_{2,3} & \leq \alpha\|A \boldsymbol{u}\|_{L^{2}}^{2}+C_{\alpha} \varepsilon^{4 \delta / 3-2}\|\boldsymbol{u}\|_{L^{2}}^{4 / 3}\left(\varepsilon^{(1-\delta) / 2}|\boldsymbol{\nu}|_{\star}^{-1 / 2}\left\|A^{1 / 2} \boldsymbol{u}\right\|_{L^{2}}\right)^{2 / 3}\left\|A^{1 / 2} \boldsymbol{u}\right\|_{L^{2}}^{4 / 3} \\
& =\alpha\|A \boldsymbol{u}\|_{L^{2}}^{2}+C_{\alpha} \varepsilon^{\delta-5 / 3}|\boldsymbol{\nu}|_{\star}^{-1 / 3}\|\boldsymbol{u}\|_{L^{2}}^{4 / 3}\left\|A^{1 / 2} \boldsymbol{u}\right\|_{L^{2}}^{2} .
\end{aligned}
$$

Since $\delta \geq 2 / 3$,

$$
J_{2,3} \leq \alpha\|A \boldsymbol{u}\|_{L^{2}}^{2}+C_{\alpha} \varepsilon^{-1}|\boldsymbol{\nu}|_{\star}^{-1 / 3}\|\boldsymbol{u}\|_{L^{2}}^{4 / 3}\left\|A^{1 / 2} \boldsymbol{u}\right\|_{L^{2}}^{2} .
$$

Therefore we obtain

$$
\begin{aligned}
\left|J_{2}\right| \leq & 2 \alpha\|A \boldsymbol{u}\|_{L^{2}}^{2}+C \varepsilon^{1 / 2}|\boldsymbol{\nu}|_{\star}^{-3 / 2}\left\|A^{1 / 2} \boldsymbol{u}\right\|_{L^{2}}\|A \boldsymbol{u}\|_{L^{2}}^{2} \\
& +C_{\alpha} \varepsilon^{-1}\left(|\boldsymbol{\nu}|_{\star}^{-1 / 3}\|\boldsymbol{u}\|_{L^{2}}^{4 / 3}+|\boldsymbol{\nu}|_{\star}^{-1}\|\boldsymbol{u}\|_{L^{2}}^{2}\right)\left\|A^{1 / 2} \boldsymbol{u}\right\|_{L^{2}}^{2} .
\end{aligned}
$$

Estimate of $J_{3}$. We have from Lemma 3.8 that

$$
\begin{aligned}
J_{3}= & \int_{\boldsymbol{\Omega}^{\varepsilon}}^{\star} \boldsymbol{\nu} \boldsymbol{\Phi} \cdot(\nabla \times \boldsymbol{\omega}) d \boldsymbol{x}=\int_{\boldsymbol{\Omega}^{\varepsilon}}^{\star} \boldsymbol{\nu}(\nabla \times \boldsymbol{\Phi}) \cdot \boldsymbol{\omega} d \boldsymbol{x}-\int_{\boldsymbol{\Omega}^{\varepsilon}}^{\star} \boldsymbol{\nu}(\nabla \times \boldsymbol{\Phi}) \cdot G(\boldsymbol{u}) d \boldsymbol{x} \\
& +\int_{\boldsymbol{\Omega}^{\varepsilon}}^{\star} \boldsymbol{\nu} \boldsymbol{\Phi} \cdot(\nabla \times G(\boldsymbol{u})) d \boldsymbol{x}-2 \gamma_{0} \int_{\boldsymbol{\Gamma}_{0}}^{\star} \boldsymbol{u} \cdot S \boldsymbol{\Phi} d \boldsymbol{\sigma} \\
= & J_{3,1}+J_{3,2}+J_{3,3}+J_{3,4} .
\end{aligned}
$$

- We estimate $J_{3,3}$ first. By Lemma 3.7, Hölder's inequality, estimate (5.4) and Lemma 4.2,

$$
\begin{aligned}
\left|J_{3,3}\right| & \leq C|\boldsymbol{\nu}|_{\star}\|\boldsymbol{\Phi}\|_{L^{2}}\left(\varepsilon^{\delta}\|\boldsymbol{u}\|_{H^{1}}+\varepsilon^{\delta-1}\|\boldsymbol{u}\|_{L^{2}}\right) \\
& \leq C|\boldsymbol{\nu}|_{\star}\left(\varepsilon^{-1 / 2}|\boldsymbol{\nu}|_{\star}^{-1}\|\boldsymbol{u}\|_{L^{2}}^{1 / 2}\left\|A^{1 / 2} \boldsymbol{u}\right\|_{L^{2}}\|A \boldsymbol{u}\|_{L^{2}}^{1 / 2}\right)\left(\varepsilon^{\delta}|\boldsymbol{\nu}|_{\star}^{-1 / 2}\left\|A^{1 / 2} \boldsymbol{u}\right\|_{L^{2}}+\varepsilon^{\delta-1}\|\boldsymbol{u}\|_{L^{2}}\right) \\
& \leq \varepsilon^{\delta-1 / 2}|\boldsymbol{\nu}|_{\star}^{-1 / 2}\|\boldsymbol{u}\|_{L^{2}}^{1 / 2}\left\|A^{1 / 2} \boldsymbol{u}\right\|_{L^{2}}^{2}\|A \boldsymbol{u}\|_{L^{2}}^{1 / 2}+\varepsilon^{\delta-3 / 2}\|\boldsymbol{u}\|_{L^{2}}^{3 / 2}\left\|A^{1 / 2} \boldsymbol{u}\right\|_{L^{2}}\|A \boldsymbol{u}\|_{L^{2}}^{1 / 2} \\
& \leq C\left(J_{2,2}+J_{2,3}\right) .
\end{aligned}
$$


Using above estimates of $J_{2,2}$ and $J_{2,3}$ with an adjustment of $\alpha$ and $C_{\alpha}$ to compensate the factor $C$, we obtain

$$
\left|J_{3,3}\right| \leq 2 \alpha\|A \boldsymbol{u}\|_{L^{2}}^{2}+C_{\alpha} \varepsilon^{-1}\left(|\boldsymbol{\nu}|_{\star}^{-1 / 3}\|\boldsymbol{u}\|_{L^{2}}^{4 / 3}+|\boldsymbol{\nu}|_{\star}^{-1}\|\boldsymbol{u}\|_{L^{2}}^{2}\right)\left\|A^{1 / 2} \boldsymbol{u}\right\|_{L^{2}}^{2} .
$$

- We estimate $J_{3,2}$ next. This is similar to the corresponding estimate in [8]. Note that

$$
\nabla \times \boldsymbol{\Phi}=(\boldsymbol{\omega} \cdot \nabla) \boldsymbol{v}-(\boldsymbol{v} \cdot \nabla) \boldsymbol{\omega}+\boldsymbol{\omega}(\nabla \cdot \boldsymbol{v})-\boldsymbol{v}(\nabla \cdot \boldsymbol{\omega}) .
$$

Since $\boldsymbol{v}$ and $\boldsymbol{\omega}$ are divergence-free, we have

$$
|\nabla \times \boldsymbol{\Phi}|=|(\boldsymbol{\omega} \cdot \nabla) \boldsymbol{v}-(\boldsymbol{v} \cdot \nabla) \boldsymbol{\omega}| \leq C\left(|\nabla \boldsymbol{v}||\nabla \boldsymbol{u}|+|\boldsymbol{v}|\left|\nabla^{2} \boldsymbol{u}\right|\right) .
$$

It follows from (3.51) and (5.8) that

$$
\left|J_{3,2}\right| \leq C|\boldsymbol{\nu}|_{\star} \int_{\boldsymbol{\Omega}^{\varepsilon}}^{\star}|\nabla \times \boldsymbol{\Phi}| \varepsilon^{\delta}|\boldsymbol{u}| d \boldsymbol{x} \leq C \varepsilon^{\delta}|\boldsymbol{\nu}|_{\star} \int_{\boldsymbol{\Omega}^{\varepsilon}}^{\star}\left(\left|\nabla \boldsymbol{v}\|\nabla \boldsymbol{u}|+| \boldsymbol{v}\| \nabla^{2} u\right|\right)|\boldsymbol{u}| d \boldsymbol{x} .
$$

For the last integral, we apply (4.45), Hölder's inequality and (A.8) to obtain

$$
\begin{aligned}
& \varepsilon^{\delta}|\boldsymbol{\nu}|_{\star} \int_{\boldsymbol{\Omega}^{\varepsilon}}^{\star}|\nabla \boldsymbol{v}\|\boldsymbol{u}\| \nabla \boldsymbol{u}| d \boldsymbol{x} \leq C \varepsilon^{\delta}|\boldsymbol{\nu}|_{\star} \int_{\boldsymbol{\Omega}^{\varepsilon}}^{\star}\left(\left|\nabla_{2} \overline{\boldsymbol{v}}\right|+|\overline{\boldsymbol{v}}|\right)|\boldsymbol{u} \| \nabla \boldsymbol{u}| d \boldsymbol{x} \\
& \leq C \varepsilon^{\delta}|\boldsymbol{\nu}|_{\star}\left\|\left(\left|\nabla_{2} \overline{\boldsymbol{v}}\right|+|\overline{\boldsymbol{v}}|\right)|\boldsymbol{u}|\right\|_{L^{2}}\|\nabla u\|_{L^{2}} \\
& \leq C \varepsilon^{\delta}|\boldsymbol{\nu}|_{\star}\left(\varepsilon^{-1 / 2}\|\overline{\boldsymbol{v}}\|_{H^{1}}^{1 / 2}\|\overline{\boldsymbol{v}}\|_{H^{2}}^{1 / 2}\|\boldsymbol{u}\|_{L^{2}}^{1 / 2}\|\boldsymbol{u}\|_{H^{1}}^{1 / 2}\right)\|\boldsymbol{u}\|_{H^{1}} \\
& \leq C \varepsilon^{\delta-1 / 2}|\boldsymbol{\nu}|_{\star}\|\boldsymbol{u}\|_{L^{2}}^{1 / 2}\|\boldsymbol{u}\|_{H^{1}}^{2}\|\boldsymbol{u}\|_{H^{2}}^{1 / 2},
\end{aligned}
$$

and then by Lemma 4.2(i) and Corollary 4.5,

$$
\begin{aligned}
\varepsilon^{\delta}|\boldsymbol{\nu}|_{\star} \int_{\boldsymbol{\Omega}^{\varepsilon}}^{\star}|\nabla \boldsymbol{v}\|\boldsymbol{u}\| \nabla \boldsymbol{u}| d \boldsymbol{x} & \leq C \varepsilon^{\delta-1 / 2}|\boldsymbol{\nu}|_{\star}^{-1 / 2}\|\boldsymbol{u}\|_{L^{2}}^{1 / 2}\left\|A^{1 / 2} \boldsymbol{u}\right\|_{L^{2}}^{2}\|A \boldsymbol{u}\|_{L^{2}}^{1 / 2} \\
& \leq C J_{2,2} \leq C J_{0}
\end{aligned}
$$

Similarly,

$$
\begin{aligned}
& \varepsilon^{\delta}|\boldsymbol{\nu}|_{\star} \int_{\boldsymbol{\Omega}^{\varepsilon}}^{\star}\left|\boldsymbol{v}\|\boldsymbol{u}\| \nabla^{2} u\right| d \boldsymbol{x} \\
& \leq C \varepsilon^{\delta}|\boldsymbol{\nu}|_{\star} \int_{\boldsymbol{\Omega}^{\varepsilon}}^{\star}\left|\overline{\boldsymbol{v}}\|\boldsymbol{u}\| \nabla^{2} \boldsymbol{u}\right| d \boldsymbol{x} \leq C \varepsilon^{\delta}|\boldsymbol{\nu}|_{\star}\||\overline{\boldsymbol{v}}||\boldsymbol{u}|\|_{L^{2}}\left\|\nabla^{2} u\right\|_{L^{2}} \\
& \leq C \varepsilon^{\delta-1 / 2}|\boldsymbol{\nu}|_{\star}\|\boldsymbol{u}\|_{L^{2}}\|\boldsymbol{u}\|_{H^{1}}\|\boldsymbol{u}\|_{H^{2}} \leq C \varepsilon^{\delta-1 / 2}|\boldsymbol{\nu}|_{\star}^{-1 / 2}\|\boldsymbol{u}\|_{L^{2}}\left\|A^{1 / 2} \boldsymbol{u}\right\|_{L^{2}}\|A \boldsymbol{u}\|_{L^{2}} \\
& \leq C J_{0} .
\end{aligned}
$$

Therefore, by (5.5),

$$
\left|J_{3,2}\right| \leq 2 \alpha\|A \boldsymbol{u}\|_{L^{2}}^{2}+C_{\alpha} \varepsilon^{-1}|\boldsymbol{\nu}|_{\star}^{-1}\|\boldsymbol{u}\|_{L^{2}}^{2}\left\|A^{1 / 2} \boldsymbol{u}\right\|_{L^{2}}^{2} .
$$

- We estimate $J_{3,1}$ by following calculations for the same term in Proposition 6.1 of [8] with some alterations. We have

$$
J_{3,1}=\int_{\boldsymbol{\Omega}^{\varepsilon}}^{\star} \boldsymbol{\nu}(\boldsymbol{\omega} \cdot \nabla \boldsymbol{v}) \cdot \boldsymbol{\omega} d \boldsymbol{x}-\int_{\boldsymbol{\Omega}^{\varepsilon}}^{\star} \boldsymbol{\nu}(\boldsymbol{v} \cdot \nabla) \boldsymbol{\omega} \cdot \boldsymbol{\omega} d \boldsymbol{x} .
$$


It is well-known that the second integral vanishes. Hence

$$
\begin{aligned}
J_{3,1} & =\int_{\boldsymbol{\Omega}^{\varepsilon}}^{\star} \boldsymbol{\nu}(\boldsymbol{\omega} \cdot \nabla \boldsymbol{v}) \cdot \boldsymbol{\omega} d \boldsymbol{x} \\
& =\int_{\boldsymbol{\Omega}^{\varepsilon}}^{\star} \boldsymbol{\nu}((\nabla \times \boldsymbol{v}) \cdot \nabla \boldsymbol{v}) \cdot \boldsymbol{\omega} d \boldsymbol{x}+\int_{\boldsymbol{\Omega}^{\varepsilon}}^{\star} \boldsymbol{\nu}((\nabla \times \boldsymbol{w}) \cdot \nabla \boldsymbol{v}) \cdot \boldsymbol{\omega} d \boldsymbol{x} \\
& =J_{3,1}^{(1)}+J_{3,1}^{(2)} .
\end{aligned}
$$

For $J_{3,1}^{(1)}$, we have exactly the same as in [8] and then use relations (4.24) and (4.33),

$$
\begin{aligned}
& \left|J_{3,1}^{(1)}\right| \leq C \varepsilon^{-1 / 2}|\boldsymbol{\nu}|_{\star}\|\boldsymbol{u}\|_{L^{2}}\|\boldsymbol{u}\|_{H^{1}}\|\boldsymbol{u}\|_{H^{2}}+C \varepsilon^{1 / 2}|\boldsymbol{\nu}|_{\star}\|\boldsymbol{u}\|_{H^{1}}^{2}\|\boldsymbol{u}\|_{H^{2}} \\
& \leq C \varepsilon^{-1 / 2}|\boldsymbol{\nu}|_{\star}^{-1 / 2}\|\boldsymbol{u}\|_{L^{2}}\left\|A^{1 / 2} \boldsymbol{u}\right\|_{L^{2}}\|A \boldsymbol{u}\|_{L^{2}}+C \varepsilon^{1 / 2}|\boldsymbol{\nu}|_{\star}^{-1}\left\|A^{1 / 2} \boldsymbol{u}\right\|_{L^{2}}^{2}\|A \boldsymbol{u}\|_{L^{2}} \\
& \leq C J_{0}+C \varepsilon^{1 / 2}|\boldsymbol{\nu}|_{\star}^{-3 / 2}\left\|A^{1 / 2} \boldsymbol{u}\right\|_{L^{2}}\|A \boldsymbol{u}\|_{L^{2}}^{2} .
\end{aligned}
$$

Thus, by (5.5),

$$
\left|J_{3,1}^{(1)}\right| \leq \alpha\|A \boldsymbol{u}\|_{L^{2}}^{2}+C_{\alpha} \varepsilon^{-1}|\boldsymbol{\nu}|_{\star}^{-1}\|\boldsymbol{u}\|_{L^{2}}^{2}\left\|A^{1 / 2} \boldsymbol{u}\right\|_{L^{2}}^{2}+C \varepsilon^{1 / 2}|\boldsymbol{\nu}|_{\star}^{-3 / 2}\left\|A^{1 / 2} \boldsymbol{u}\right\|_{L^{2}}\|A \boldsymbol{u}\|_{L^{2}}^{2}
$$

For $J_{3,1}^{(2)}$, letting $\boldsymbol{v}=\overline{\boldsymbol{v}}+\boldsymbol{v}_{3} e_{3}$, we have

$$
\begin{aligned}
J_{3,1}^{(2)}= & \int_{\boldsymbol{\Omega}^{\varepsilon}}^{\star} \boldsymbol{\nu}(\nabla \times \boldsymbol{w} \cdot \nabla \overline{\boldsymbol{v}}) \cdot \nabla \times\left(\overline{\boldsymbol{v}}+\boldsymbol{v}_{3} e_{3}+\boldsymbol{w}\right) d \boldsymbol{x}+\int_{\boldsymbol{\Omega}^{\varepsilon}}^{\star} \boldsymbol{\nu}\left(\nabla \times \boldsymbol{w} \cdot \nabla\left(\boldsymbol{v}_{3} e_{3}\right)\right) \cdot \boldsymbol{\omega} d \boldsymbol{x} \\
= & \int_{\boldsymbol{\Omega}^{\varepsilon}}^{\star} \boldsymbol{\nu}\left(\nabla \times \boldsymbol{w} \cdot \nabla_{2} \overline{\boldsymbol{v}}\right) \cdot \nabla \times\left(\boldsymbol{v}_{3} e_{3}\right) d \boldsymbol{x}+\int_{\boldsymbol{\Omega}^{\varepsilon}}^{\star} \boldsymbol{\nu}\left(\nabla \times \boldsymbol{w} \cdot \nabla_{2} \overline{\boldsymbol{v}}\right) \cdot \nabla \times \boldsymbol{w} d \boldsymbol{x} \\
& +\int_{\boldsymbol{\Omega}^{\varepsilon}}^{\star} \boldsymbol{\nu}\left(\nabla \times \boldsymbol{w} \cdot \nabla\left(\boldsymbol{v}_{3} e_{3}\right)\right) \cdot \boldsymbol{\omega} d \boldsymbol{x} \\
= & K_{1}^{\prime}+K_{2}^{\prime}+K_{3}^{\prime} .
\end{aligned}
$$

As in [8],

$$
\left|K_{1}^{\prime}\right| \leq C \varepsilon^{1 / 2}|\boldsymbol{\nu}|_{\star}\|\boldsymbol{u}\|_{H^{1}}\|\boldsymbol{u}\|_{H^{2}}\|\nabla \boldsymbol{w}\|_{L^{2}} \leq C \varepsilon^{1 / 2}|\boldsymbol{\nu}|_{\star}\|\boldsymbol{u}\|_{H^{1}}\|\boldsymbol{u}\|_{H^{2}}^{2}
$$

hence

$$
\left|K_{1}^{\prime}\right| \leq C \varepsilon^{1 / 2}|\boldsymbol{\nu}|_{\star}^{-3 / 2}\left\|A^{1 / 2} \boldsymbol{u}\right\|_{L^{2}}\|A \boldsymbol{u}\|_{L^{2}}^{2}
$$

Applying Hölder's inequality and (A.8) yields

$$
\begin{aligned}
\left|K_{2}^{\prime}\right| & \leq C|\boldsymbol{\nu}|_{\star}\left\|\left|\nabla_{2} \overline{\boldsymbol{v}}\right| \cdot|\nabla \boldsymbol{w}|\right\|_{L^{2}}\|\nabla \boldsymbol{w}\|_{L^{2}} \\
& \leq C|\boldsymbol{\nu}|_{\star}\left(\varepsilon^{-1 / 2}\|\boldsymbol{v}\|_{H^{1}}^{1 / 2}\|\boldsymbol{v}\|_{H^{2}}^{1 / 2}\|\nabla \boldsymbol{w}\|_{L^{2}}^{1 / 2}\|\nabla \boldsymbol{w}\|_{H^{1}}^{1 / 2}\right)\|\nabla \boldsymbol{w}\|_{L^{2}} \\
& \leq C \varepsilon^{-1 / 2}|\boldsymbol{\nu}|_{\star}\|\boldsymbol{u}\|_{H^{1}}^{1 / 2}\|\nabla \boldsymbol{w}\|_{L^{2}}^{3 / 2}\|\boldsymbol{u}\|_{H^{2}} \\
& \leq C \varepsilon^{-1 / 2}|\boldsymbol{\nu}|_{\star}\|\boldsymbol{u}\|_{H^{1}}\|\nabla \boldsymbol{w}\|_{L^{2}}\|\boldsymbol{u}\|_{H^{2}}
\end{aligned}
$$


Estimating $\|\nabla \boldsymbol{w}\|_{L^{2}}$ by (4.51), and also using (4.24) and (4.33) for the $H^{1}$ and $H^{2}$ norms, we obtain

$$
\begin{aligned}
\left|K_{2}^{\prime}\right| & \leq C \varepsilon^{-1 / 2}|\boldsymbol{\nu}|_{\star}\left(|\boldsymbol{\nu}|_{\star}^{-1 / 2}\left\|A^{1 / 2} \boldsymbol{u}\right\|_{L^{2}}\right)\left(\varepsilon|\boldsymbol{\nu}|_{\star}^{-1}\|A \boldsymbol{u}\|_{L^{2}}\right)\left(|\boldsymbol{\nu}|_{\star}^{-1}\|A \boldsymbol{u}\|_{L^{2}}\right) \\
& \leq C \varepsilon^{1 / 2}|\boldsymbol{\nu}|_{\star}^{-3 / 2}\left\|A^{1 / 2} \boldsymbol{u}\right\|_{L^{2}}\|A \boldsymbol{u}\|_{L^{2}}^{2} .
\end{aligned}
$$

For $K_{3}^{\prime}$, by estimates in (4.44),

$$
\left|K_{3}^{\prime}\right| \leq C|\boldsymbol{\nu}|_{\star} \int_{\boldsymbol{\Omega}^{\varepsilon}}^{\star}|\nabla \boldsymbol{w}|\left(\varepsilon\left|\nabla_{2} \overline{\boldsymbol{v}}\right|+|\overline{\boldsymbol{v}}|\right)|\nabla \boldsymbol{u}| d \boldsymbol{x} .
$$

Using (A.8), we estimate

$$
\begin{aligned}
& \varepsilon|\boldsymbol{\nu}|_{\star} \int_{\boldsymbol{\Omega}^{\varepsilon}}^{\star}\left(\left|\nabla \boldsymbol{w} \| \nabla_{2} \overline{\boldsymbol{v}}\right|\right)|\nabla \boldsymbol{u}| d \boldsymbol{x} \leq C \varepsilon|\boldsymbol{\nu}|_{\star}\left(\varepsilon^{-1 / 2}\|\nabla \boldsymbol{w}\|_{L^{2}}^{1 / 2}\|\nabla \boldsymbol{w}\|_{H^{1}}^{1 / 2}\|\boldsymbol{v}\|_{H^{1}}^{1 / 2}\|\boldsymbol{v}\|_{H^{2}}^{1 / 2}\right)\|\boldsymbol{u}\|_{H^{1}} \\
& \leq C \varepsilon^{1 / 2}|\boldsymbol{\nu}|_{\star}\|\boldsymbol{u}\|_{H^{1}}^{2}\|\boldsymbol{u}\|_{H^{2}} \leq C \varepsilon^{1 / 2}|\boldsymbol{\nu}|_{\star}\|\boldsymbol{u}\|_{H^{1}}\|\boldsymbol{u}\|_{H^{2}}^{2} \\
& \leq C \varepsilon^{1 / 2}|\boldsymbol{\nu}|_{\star}^{-3 / 2}\left\|A^{1 / 2} \boldsymbol{u}\right\|_{L^{2}}\|A \boldsymbol{u}\|_{L^{2}}^{2} .
\end{aligned}
$$

By the 2D Agmon inequality (A.7) applied to $\overline{\boldsymbol{v}}$, we have

$$
\begin{aligned}
& |\boldsymbol{\nu}|_{\star} \int_{\boldsymbol{\Omega}^{\varepsilon}}^{\star}|\nabla \boldsymbol{w}\|\overline{\boldsymbol{v}}\| \nabla \boldsymbol{u}| d \boldsymbol{x} \leq|\boldsymbol{\nu}|_{\star}\|\overline{\boldsymbol{v}}\|_{L^{\infty}}\|\nabla \boldsymbol{w}\|_{L^{2}}\|\nabla u\|_{L^{2}} \\
& \leq C|\boldsymbol{\nu}|_{\star}\left(\varepsilon^{-1 / 2}\|\boldsymbol{u}\|_{L^{2}}^{1 / 2}\|\boldsymbol{u}\|_{H^{2}}^{1 / 2}\right)\|\boldsymbol{u}\|_{H^{1}}^{2} \\
& \leq C \varepsilon^{-1 / 2}|\boldsymbol{\nu}|_{\star}^{-1 / 2}\|\boldsymbol{u}\|_{L^{2}}^{1 / 2}\left\|A^{1 / 2} \boldsymbol{u}\right\|_{L^{2}}^{2}\|A \boldsymbol{u}\|_{L^{2}}^{1 / 2} \leq C J_{2,2} \leq C J_{0} .
\end{aligned}
$$

Combining with (5.5), we obtain

$$
\begin{aligned}
\left|K_{3}^{\prime}\right| \leq & \alpha\|A \boldsymbol{u}\|_{L^{2}}^{2}+C \varepsilon^{1 / 2}|\boldsymbol{\nu}|_{\star}^{-3 / 2}\left\|A^{1 / 2} \boldsymbol{u}\right\|_{L^{2}}\|A \boldsymbol{u}\|_{L^{2}}^{2} \\
& +C_{\alpha} \varepsilon^{-1}|\boldsymbol{\nu}|_{\star}^{-1}\|\boldsymbol{u}\|_{L^{2}}^{2}\left\|A^{1 / 2} \boldsymbol{u}\right\|_{L^{2}}^{2} .
\end{aligned}
$$

Gathering the estimates of $K_{1}^{\prime}, K_{2}^{\prime}$ and $K_{3}^{\prime}$, we have

$$
\begin{aligned}
\left|J_{3,1}^{(2)}\right| \leq & \left|K_{1}^{\prime}\right|+\left|K_{2}^{\prime}\right|+\left|K_{3}^{\prime}\right| \\
\leq & \alpha\|A \boldsymbol{u}\|_{L^{2}}^{2}+C \varepsilon^{1 / 2}|\boldsymbol{\nu}|_{\star}^{-3 / 2}\left\|A^{1 / 2} \boldsymbol{u}\right\|_{L^{2}}\|A \boldsymbol{u}\|_{L^{2}}^{2} \\
& +C_{\alpha} \varepsilon^{-1}|\boldsymbol{\nu}|_{\star}^{-1}\|\boldsymbol{u}\|_{L^{2}}^{2}\left\|A^{1 / 2} \boldsymbol{u}\right\|_{L^{2}}^{2} .
\end{aligned}
$$

Summing up,

$$
\begin{aligned}
\left|J_{3,1}\right| \leq & \left|J_{3,1}^{(1)}\right|+\left|J_{3,1}^{(2)}\right| \\
\leq & 2 \alpha\|A \boldsymbol{u}\|_{L^{2}}^{2}+C \varepsilon^{1 / 2}|\boldsymbol{\nu}|_{\star}^{-3 / 2}\left\|A^{1 / 2} \boldsymbol{u}\right\|_{L^{2}}\|A \boldsymbol{u}\|_{L^{2}}^{2} \\
& +C_{\alpha} \varepsilon^{-1}|\boldsymbol{\nu}|_{\star}^{-1}\|\boldsymbol{u}\|_{L^{2}}^{2}\left\|A^{1 / 2} \boldsymbol{u}\right\|_{L^{2}}^{2} .
\end{aligned}
$$


- Finally, we estimate the boundary term $J_{3,4}$. By (2.17), (5.1) and (2.18), we have

$$
\begin{aligned}
\left|J_{3,4}\right| & \leq 2 \gamma_{0} \int_{\Gamma_{0}}^{\star}|\boldsymbol{u} \cdot(S \boldsymbol{v} \times S \boldsymbol{\omega})| d \boldsymbol{\sigma} \leq C \varepsilon|\boldsymbol{\nu}|_{\star} \int_{\Gamma_{0}}|\boldsymbol{u}|_{\star}|\boldsymbol{v}|_{\star}|\boldsymbol{\omega}|_{\star} d \sigma \\
& \leq C \varepsilon|\boldsymbol{\nu}|_{\star} \int_{\Gamma_{0}}|\boldsymbol{v}|_{\star}|\boldsymbol{u}|_{\star}|\nabla \boldsymbol{u}|_{\star} d \sigma .
\end{aligned}
$$

For an estimation of $\varepsilon \int_{\Gamma_{0}}|\boldsymbol{v}|_{\star}|\boldsymbol{u}|_{\star}|\nabla \boldsymbol{u}|_{\star} d \sigma$, we use the calculations in estimating the term $I_{3}$ on page 595 , from line 7 to line 23 , of [9]. We obtain

$$
\begin{aligned}
\left|J_{3,4}\right| \leq & C|\boldsymbol{\nu}|_{\star} \varepsilon^{-1 / 2}\|\boldsymbol{u}\|_{L^{2}}\|\boldsymbol{u}\|_{H^{1}}^{2}+C|\boldsymbol{\nu}|_{\star} \varepsilon\|\boldsymbol{u}\|_{L^{2}}^{1 / 2}\|\boldsymbol{u}\|_{H^{1}}^{3 / 2}\|\boldsymbol{u}\|_{H^{2}} \\
& +C|\boldsymbol{\nu}|_{\star} \varepsilon^{1 / 2}\|\boldsymbol{u}\|_{L^{2}}\|\boldsymbol{u}\|_{H^{1}}\|\boldsymbol{u}\|_{H^{2}}+C|\boldsymbol{\nu}|_{\star} \varepsilon^{2}\|\boldsymbol{u}\|_{L^{2}}^{1 / 2}\|\boldsymbol{u}\|_{H^{1}}^{1 / 2}\|\boldsymbol{u}\|_{H^{2}}^{2} \\
\leq & C|\boldsymbol{\nu}|_{\star} \varepsilon^{-1 / 2}\|\boldsymbol{u}\|_{L^{2}}\|\boldsymbol{u}\|_{H^{1}}^{2}+C|\boldsymbol{\nu}|_{\star} \varepsilon^{1 / 2}\|\boldsymbol{u}\|_{H^{1}}\|\boldsymbol{u}\|_{H^{2}}^{2}
\end{aligned}
$$

Hence, by (4.24) and (4.33),

$$
\left|J_{3,4}\right| \leq C \varepsilon^{-1}\|\boldsymbol{u}\|_{L^{2}}\left\|A^{1 / 2} \boldsymbol{u}\right\|_{L^{2}}^{2}+C \varepsilon^{1 / 2}|\boldsymbol{\nu}|_{\star}^{-3 / 2}\left\|A^{1 / 2} \boldsymbol{u}\right\|_{L^{2}}\|A \boldsymbol{u}\|_{L^{2}}^{2} .
$$

It follows from estimates (5.10), (5.9), (5.7) and (5.11) that

$$
\begin{aligned}
\left|J_{3}\right| \leq & \left|J_{3,1}\right|+\left|J_{3,2}\right|+\left|J_{3,3}\right|+\left|J_{3,4}\right| \\
\leq & 6 \alpha\|A \boldsymbol{u}\|_{L^{2}}^{2}+C \varepsilon^{1 / 2}|\boldsymbol{\nu}|_{\star}^{-3 / 2}\left\|A^{1 / 2} \boldsymbol{u}\right\|_{L^{2}}\|A \boldsymbol{u}\|_{L^{2}}^{2} \\
& +C_{\alpha} \varepsilon^{-1}\left(|\boldsymbol{\nu}|_{\star}^{-1}\|\boldsymbol{u}\|_{L^{2}}^{2}+|\boldsymbol{\nu}|_{\star}^{-1 / 3}\|\boldsymbol{u}\|_{L^{2}}^{4 / 3}+\|\boldsymbol{u}\|_{L^{2}}\right)\left\|A^{1 / 2} \boldsymbol{u}\right\|_{L^{2}}^{2} .
\end{aligned}
$$

Summing up the estimates (5.3), (5.6) and (5.12) for $J_{1}, J_{2}$ and $J_{3}$, respectively, we obtain

$$
\begin{aligned}
|I| \leq & 8 \alpha\|A \boldsymbol{u}\|_{L^{2}}^{2}+C \varepsilon^{1 / 2}|\boldsymbol{\nu}|_{\star}^{-3 / 2}\left\|A^{1 / 2} \boldsymbol{u}\right\|_{L^{2}}\|A \boldsymbol{u}\|_{L^{2}}^{2} \\
& +C_{\alpha} \varepsilon^{-1}\left(|\boldsymbol{\nu}|_{\star}^{-1}\|\boldsymbol{u}\|_{L^{2}}^{2}+|\boldsymbol{\nu}|_{\star}^{-1 / 3}\|\boldsymbol{u}\|_{L^{2}}^{4 / 3}+\|\boldsymbol{u}\|_{L^{2}}\right)\left\|A^{1 / 2} \boldsymbol{u}\right\|_{L^{2}}^{2}
\end{aligned}
$$

Using Young's inequality to estimate $|\boldsymbol{\nu}|_{\star}^{-1 / 3}\|\boldsymbol{u}\|_{L^{2}}^{4 / 3} \leq|\boldsymbol{\nu}|_{\star}^{-1}\|\boldsymbol{u}\|_{L^{2}}^{2}+\|\boldsymbol{u}\|_{L^{2}}$, and replacing $\alpha$ by $\alpha / 8$, we obtain the assertion (5.2) from (5.13).

\section{GlobAl SOLUTiOnS}

First, we state the standard local existence theorem for strong solutions of the Navier-Stokes equations. Below, we use the conventional notation $\boldsymbol{u}(t)=\boldsymbol{u}(\cdot, t)$ and $\boldsymbol{f}(t)=\boldsymbol{f}(\cdot, t)$. We recall that $\varepsilon_{*}$ is defined in Proposition 4.4.

Theorem 6.1. Assume (5.1). Suppose $\varepsilon \in\left(0, \varepsilon_{*}\right]$ and $\boldsymbol{u}^{0} \in V$ and $\boldsymbol{f} \in L^{\infty}\left((0, \infty), L^{2}\left(\boldsymbol{\Omega}^{\varepsilon}\right)^{3}\right)$. Then there exist $T>0$ and a unique solution

$$
\boldsymbol{u}(t) \in C([0, T), V) \cap L^{2}\left(0, T ; D_{A}\right)
$$

of the equation (4.19) on $(0, T)$ satisfying $\boldsymbol{u}(0)=\boldsymbol{u}^{0}$. 
Furthermore, if the maximal time interval of the above existence is $\left[0, T_{\max }\right)$ and $T_{\max }$ is finite, then

$$
\lim _{t \rightarrow T_{\max }^{-}}\left\|A^{1 / 2} \boldsymbol{u}(t)\right\|_{L^{2}}=\infty .
$$

We call the above solution $\boldsymbol{u}(t)$ a strong solution on [0,T) of the IBVP (2.9), (2.10), (2.11), (2.12), (2.13) .

We have a few observations before stating our global existence result.

Let $\boldsymbol{u}=\left(\boldsymbol{u}_{1}, \boldsymbol{u}_{2}, \boldsymbol{u}_{3}\right) \in L^{2}\left(\Omega^{\varepsilon}\right)^{3}$, we define

$$
\bar{M} \boldsymbol{u}=\left(M_{0} \boldsymbol{u}_{1}, M_{0} \boldsymbol{u}_{2}, 0\right) .
$$

One can verify that $\bar{M}$ is an orthogonal projection on $L^{2}\left(\Omega^{\varepsilon}\right)^{3}$, hence

$$
\|\boldsymbol{u}\|_{L^{2}}^{2}=\|\bar{M} \boldsymbol{u}\|_{L^{2}}^{2}+\|(I-\bar{M}) \boldsymbol{u}\|_{L^{2}}^{2} .
$$

For $\boldsymbol{u} \in V$, it follows from (A.4) and (3.21) that

$$
\|(I-\bar{M}) \boldsymbol{u}\|_{L^{2}} \leq\left\|(I-\bar{M})\left(\boldsymbol{u}_{1}, \boldsymbol{u}_{2}\right)\right\|_{L^{2}}+\left\|\boldsymbol{u}_{3}\right\|_{L^{2}} \leq C \varepsilon\|\boldsymbol{u}\|_{H^{1}},
$$

and, consequently, by (4.24)

$$
\|(I-\bar{M}) \boldsymbol{u}\|_{L^{2}} \leq C \varepsilon|\boldsymbol{\nu}|_{\star}^{-1 / 2}\left\|A^{1 / 2} \boldsymbol{u}\right\|_{L^{2}} .
$$

For a function $\boldsymbol{f} \in L^{\infty}\left(0, \infty ; L^{2}\left(\boldsymbol{\Omega}^{\varepsilon}\right)\right)$, denote

$$
\|\boldsymbol{f}\|_{L^{\infty} L^{2}}=\operatorname{ess~sup}_{t \in(0, \infty)}\|\boldsymbol{f}(t)\|_{L^{2}} .
$$

Our main result is:

Theorem 6.2. Assume (5.1). There is a positive number $\kappa>0$ such that if $\varepsilon \in\left(0, \varepsilon_{*}\right]$ and $\boldsymbol{u}^{0} \in V, \boldsymbol{f} \in L^{\infty}\left((0, \infty), L^{2}\left(\boldsymbol{\Omega}^{\varepsilon}\right)^{3}\right)$ satisfy that

$$
\begin{array}{ll}
U_{0} \stackrel{\text { def }}{=}|\boldsymbol{\nu}|_{\star}^{-2}\left\|\bar{M} \boldsymbol{u}^{0}\right\|_{L^{2}}^{2} \leq \kappa^{2}, & U_{1} \stackrel{\text { def }}{=} \varepsilon|\boldsymbol{\nu}|_{\star}^{-3}\left\|A^{1 / 2} \boldsymbol{u}^{0}\right\|_{L^{2}}^{2} \leq \kappa^{2}, \\
F_{0} \stackrel{\text { def }}{=} \varepsilon^{2-2 \delta}|\boldsymbol{\nu}|_{\star}^{-4}\|\bar{M} P \boldsymbol{f}\|_{L^{\infty} L^{2}}^{2} \leq \kappa^{2}, & F_{1} \stackrel{\text { def }}{=} \varepsilon^{2-\delta}|\boldsymbol{\nu}|_{\star}^{-4}\|(I-\bar{M}) P \boldsymbol{f}\|_{L^{\infty} L^{2}}^{2} \leq \kappa^{2}
\end{array}
$$

then the strong solution $\boldsymbol{u}(t)$ in Theorem 6.1 exists for all $t \geq 0$, i.e., $T_{\max }=\infty$.

Moreover,

$$
|\boldsymbol{\nu}|_{\star}^{-2}\|\boldsymbol{u}(t)\|_{L^{2}}^{2} \leq c^{*}\left(\Lambda_{1} e^{-c_{2}^{-2} \varepsilon^{\delta-1}|\boldsymbol{\nu}|_{\star} t}+\Lambda_{2}\right), \quad t \in[0, \infty)
$$

and

$$
\begin{array}{lll}
\varepsilon|\boldsymbol{\nu}|_{\star}^{-3}\left\|A^{1 / 2} \boldsymbol{u}(t)\right\|_{L^{2}}^{2} \leq c^{*}\left(\Lambda_{3}+\Lambda_{4}\right), & t \in\left[0, \varepsilon^{1-\delta} /|\boldsymbol{\nu}|_{\star}\right), \\
\varepsilon|\boldsymbol{\nu}|_{\star}^{-3}\left\|A^{1 / 2} \boldsymbol{u}(t)\right\|_{L^{2}}^{2} \leq c^{*}\left(\Lambda_{1} e^{-c_{2}^{-2} \varepsilon^{\delta-1}|\boldsymbol{\nu}|_{\star} t}+\Lambda_{4}\right), & t \in\left[\varepsilon^{1-\delta} /|\boldsymbol{\nu}|_{\star}, \infty\right),
\end{array}
$$

where

$$
\Lambda_{1}=U_{0}+\varepsilon U_{1}, \quad \Lambda_{2}=F_{0}+\varepsilon F_{1}, \quad \Lambda_{3}=U_{0}+U_{1}, \quad \Lambda_{4}=F_{0}+F_{1},
$$

the number $c_{2}>0$ is defined in Lemma 4.2, and $c^{*}>0$ is independent of $\varepsilon, \boldsymbol{\nu}, \kappa, \boldsymbol{u}^{0}, \boldsymbol{f}$. 
For $\boldsymbol{u} \in V$, let

$$
E_{*}(\boldsymbol{u})=\int_{\boldsymbol{\Omega}^{\varepsilon}}^{\star}|D \boldsymbol{u}|^{2} d \boldsymbol{x}+\varepsilon^{\delta} \int_{\boldsymbol{\Gamma}}^{\star}|\boldsymbol{u}|^{2} d \boldsymbol{\sigma}+\varepsilon \int_{\boldsymbol{\Gamma}_{0}}^{\star}|\boldsymbol{u}-S \boldsymbol{u}|^{2} d \boldsymbol{\sigma} .
$$

Still considering $\delta_{0}=1$, we then have

$$
C^{-1} E_{*}(\boldsymbol{u}) \leq|\boldsymbol{\nu}|_{\star}^{-1} E(\boldsymbol{u}) \leq C E_{*}(\boldsymbol{u}),
$$

or, equivalently,

$$
C^{-1} E_{*}(\boldsymbol{u}) \leq|\boldsymbol{\nu}|_{\star}^{-1}\left\|A^{1 / 2} \boldsymbol{u}\right\|_{L^{2}}^{2} \leq C E_{*}(\boldsymbol{u}) .
$$

Therefore, Theorem 6.2 can be rewritten without using the Stokes operator as:

Theorem 6.3. Suppose the assumptions in Theorem 6.2 hold with $U_{1}$ in (6.5) being redefined by

$$
U_{1} \stackrel{\text { def }}{=} \varepsilon|\boldsymbol{\nu}|_{\star}^{-2} E_{*}\left(\boldsymbol{u}^{0}\right) .
$$

Then one has (6.6) and, in place of (6.7) and (6.8),

$$
\begin{array}{lll}
\varepsilon|\boldsymbol{\nu}|_{\star}^{-2} E_{*}(\boldsymbol{u}(t)) \leq c^{*}\left(\Lambda_{3}+\Lambda_{4}\right), & & t \in\left[0, \varepsilon^{1-\delta} /|\boldsymbol{\nu}|_{\star}\right), \\
\varepsilon|\boldsymbol{\nu}|_{\star}^{-2} E_{*}(\boldsymbol{u}(t)) \leq c^{*}\left(\Lambda_{1} e^{-c_{2}^{-2} \varepsilon^{\delta-1}|\boldsymbol{\nu}|_{\star} t}+\Lambda_{4}\right), & & t \in\left[\varepsilon^{1-\delta} /|\boldsymbol{\nu}|_{\star}, \infty\right) .
\end{array}
$$

Proof of Theorem 6.2. Note from (6.3) and (6.4) that

$$
\left\|\boldsymbol{u}^{0}\right\|_{L^{2}}^{2} \leq\left\|\bar{M} \boldsymbol{u}^{0}\right\|_{L^{2}}^{2}+c_{4}^{2} \varepsilon^{2}|\boldsymbol{\nu}|_{\star}^{-1}\left\|A^{1 / 2} \boldsymbol{u}^{0}\right\|_{L^{2}}^{2} \leq C|\boldsymbol{\nu}|_{\star}^{2}\left(U_{0}+\varepsilon U_{1}\right)=C|\boldsymbol{\nu}|_{\star}^{2} \Lambda_{1} .
$$

Also,

$$
\|P \boldsymbol{f}\|_{L^{2}}^{2}=\|\bar{M} P \boldsymbol{f}\|_{L^{2}}^{2}+\|(I-\bar{M}) P \boldsymbol{f}\|_{L^{2}}^{2} \leq|\boldsymbol{\nu}|_{\star}^{4}\left(\varepsilon^{2 \delta-2} F_{0}+\varepsilon^{\delta-2} F_{1}\right) \leq \varepsilon^{\delta-2}|\boldsymbol{\nu}|_{\star}^{4} \Lambda_{4} .
$$

Obviously, from (6.9) and (6.5):

$$
\Lambda_{1}, \Lambda_{2}, \Lambda_{3}, \Lambda_{4} \leq 2 \kappa^{2}
$$

Set

$$
d=\frac{1}{16 c_{3}^{2}} \quad \text { and } \quad \kappa^{2}=\min \left\{1, \frac{d}{2}, \frac{d}{2 C_{0}}\right\}
$$

where $c_{3}>0$ is from Proposition 5.1 and $C_{0}>0$ is the constant in Lemma 6.4 below.

Let $0<\varepsilon<\varepsilon_{*} \leq 1$. We estimate $\|\boldsymbol{u}(t)\|_{L^{2}}$ first. Taking the inner product of equation (4.19) with $\boldsymbol{u}(t)$ for $t \in\left(0, T_{\max }\right)$, and applying inequalities (4.23) and (4.50), we have

$$
\begin{aligned}
& \frac{1}{2} \frac{d}{d t}\|\boldsymbol{u}\|_{L^{2}}^{2}+\left\|A^{1 / 2} \boldsymbol{u}\right\|_{L^{2}}^{2} \leq|\langle\boldsymbol{u}, \boldsymbol{f}\rangle| \leq|\langle\bar{M} \boldsymbol{u}, \bar{M} P \boldsymbol{f}\rangle|+|\langle(I-\bar{M}) \boldsymbol{u},(I-\bar{M}) P \boldsymbol{f}\rangle| \\
& \leq C \varepsilon^{(1-\delta) / 2}|\boldsymbol{\nu}|_{\star}^{-1 / 2}\left\|A^{1 / 2} \boldsymbol{u}\right\|_{L^{2}}\|\bar{M} P \boldsymbol{f}\|_{L^{2}}+C \varepsilon|\boldsymbol{\nu}|_{\star}^{-1 / 2}\left\|A^{1 / 2} \boldsymbol{u}\right\|_{L^{2}}\|(I-\bar{M}) P \boldsymbol{f}\|_{L^{2}} \\
& \leq \frac{1}{2}\left\|A^{1 / 2} \boldsymbol{u}\right\|_{L^{2}}^{2}+C \varepsilon^{1-\delta}|\boldsymbol{\nu}|_{\star}^{-1}\|\bar{M} P \boldsymbol{f}\|_{L^{2}}^{2}+C \varepsilon^{2}|\boldsymbol{\nu}|_{\star}^{-1}\|(I-\bar{M}) P \boldsymbol{f}\|_{L^{2}}^{2} \\
& \leq \frac{1}{2}\left\|A^{1 / 2} \boldsymbol{u}\right\|_{L^{2}}^{2}+C \varepsilon^{\delta-1}|\boldsymbol{\nu}|_{\star}^{3}\left(F_{0}+\varepsilon F_{1}\right) .
\end{aligned}
$$


Hence

Using (4.23) in (6.19) gives

$$
\frac{d}{d t}\|\boldsymbol{u}\|_{L^{2}}^{2}+\left\|A^{1 / 2} \boldsymbol{u}\right\|_{L^{2}}^{2} \leq C \varepsilon^{\delta-1}|\boldsymbol{\nu}|_{\star}^{3} \Lambda_{2}
$$

$$
\frac{d}{d t}\|\boldsymbol{u}\|_{L^{2}}^{2}+\frac{|\boldsymbol{\nu}|_{\star}}{c_{2}^{2} \varepsilon^{1-\delta}}\|\boldsymbol{u}\|_{L^{2}}^{2} \leq C \varepsilon^{\delta-1}|\boldsymbol{\nu}|_{\star}^{3} \Lambda_{2} .
$$

Applying Gronwall's inequality, we obtain

$$
\|\boldsymbol{u}(t)\|_{L^{2}}^{2} \leq\left\|\boldsymbol{u}^{0}\right\|_{L^{2}}^{2} e^{-c_{2}^{-2} \varepsilon^{\delta-1}|\boldsymbol{\nu}|_{\star} t}+C|\boldsymbol{\nu}|_{\star}^{2} \Lambda_{2}, \quad t \in\left[0, T_{\max }\right) .
$$

Using (6.15) to estimate $\left\|\boldsymbol{u}^{0}\right\|_{L^{2}}^{2}$, we have

$$
\|\boldsymbol{u}(t)\|_{L^{2}}^{2} \leq C_{1}|\boldsymbol{\nu}|_{\star}^{2}\left(\Lambda_{1} e^{-c_{2}^{-2} \varepsilon^{\delta-1}|\boldsymbol{\nu}|_{\star} t}+\Lambda_{2}\right), \quad t \in\left[0, T_{\max }\right),
$$

and consequently, by $(6.17)$

$$
\|\boldsymbol{u}(t)\|_{L^{2}}^{2} \leq C_{1}|\boldsymbol{\nu}|_{\star}^{2}\left(\Lambda_{1}+\Lambda_{2}\right) \leq 4 C_{1}|\boldsymbol{\nu}|_{\star}^{2} \kappa^{2}, \quad t \in\left[0, T_{\max }\right) .
$$

For any number $\theta \in\left[0, \varepsilon^{1-\delta} /|\boldsymbol{\nu}|_{\star}\right] \cap\left[0, T_{\max }\right)$ and $t \in\left[0, T_{\max }-\theta\right)$, integrating (6.19) from $t$ to $t+\theta$ and using (6.20) yield

$$
\begin{aligned}
\int_{t}^{t+\theta}\left\|A^{1 / 2} \boldsymbol{u}(\tau)\right\|_{L^{2}}^{2} d \tau & \leq\|\boldsymbol{u}(t)\|_{L^{2}}^{2}+C \varepsilon^{\delta-1}|\boldsymbol{\nu}|_{\star}^{3} \int_{t}^{t+\theta} \Lambda_{2} d \tau \\
& \leq C|\boldsymbol{\nu}|_{\star}^{2}\left(\Lambda_{1} e^{-c_{2}^{-2} \varepsilon^{\delta-1}|\boldsymbol{\nu}|_{\star} t}+\Lambda_{2}\right)+C \varepsilon^{\delta-1}|\boldsymbol{\nu}|_{\star}^{3} \theta \Lambda_{2} .
\end{aligned}
$$

Hence we have

$$
\int_{t}^{t+\theta}\left\|A^{1 / 2} \boldsymbol{u}(\tau)\right\|_{L^{2}}^{2} d \tau \leq C_{2}|\boldsymbol{\nu}|_{\star}^{2}\left(\Lambda_{1} e^{-c_{2}^{-2} \varepsilon^{\delta-1}|\boldsymbol{\nu}|_{\star} t}+\Lambda_{2}\right), \quad t \in\left[0, T_{\max }-\theta\right) .
$$

and, again, by (6.17)

$$
\int_{t}^{t+\theta}\left\|A^{1 / 2} \boldsymbol{u}(\tau)\right\|_{L^{2}}^{2} d \tau \leq 4 C_{2}|\boldsymbol{\nu}|_{\star}^{2} \kappa^{2}, \quad t \in\left[0, T_{\max }-\theta\right) .
$$

Next, we estimate $\left\|A^{1 / 2} \boldsymbol{u}(t)\right\|_{L^{2}}$. The initial data satisfies

$$
\varepsilon|\boldsymbol{\nu}|_{\star}^{-3}\left\|A^{1 / 2} u^{0}\right\|_{L^{2}}^{2}=U_{1} \leq \kappa^{2} \leq \frac{d}{2} .
$$

We claim that

$$
\varepsilon|\boldsymbol{\nu}|_{\star}^{-3}\left\|A^{1 / 2} \boldsymbol{u}(t)\right\|_{L^{2}}^{2} \leq d \text { for all } t \in\left[0, T_{\max }\right) .
$$

Suppose (6.24) is false. By the continuity of $\boldsymbol{u}(t)$ in $V$ for $t \in[0, T)$, there is $T \in\left(0, T_{\max }\right)$ such that

$$
\varepsilon|\boldsymbol{\nu}|_{\star}^{-3}\left\|A^{1 / 2} \boldsymbol{u}(t)\right\|_{L^{2}}^{2}<d \quad \text { for all } t<T
$$

and

$$
\varepsilon|\boldsymbol{\nu}|_{\star}^{-3}\left\|A^{1 / 2} \boldsymbol{u}(T)\right\|_{L^{2}}^{2}=d .
$$

We apply Lemma 6.4 below and have from its conclusion (6.27) that

$$
\varepsilon|\boldsymbol{\nu}|_{\star}^{-3}\left\|A^{1 / 2} \boldsymbol{u}(t)\right\|_{L^{2}}^{2} \leq C_{0} \kappa^{2} \leq \frac{d}{2} \quad \text { for all } t \in[0, T) .
$$


Taking $t \rightarrow T^{-}$, we have

$$
\varepsilon|\boldsymbol{\nu}|_{\star}^{-3}\left\|A^{1 / 2} \boldsymbol{u}(T)\right\|_{L^{2}}^{2} \leq \frac{d}{2}<d,
$$

which contradicts (6.25). Therefore (6.24) must hold true. As a consequence of (6.24), the norm $\left\|A^{1 / 2} \boldsymbol{u}(t)\right\|_{L^{2}}$ is bounded on $\left[0, T_{\max }\right)$, which implies $T_{\max }=\infty$, by virtue of Theorem 6.1 .

The estimate (6.6) follows (6.20). Since (6.29) and (6.32) in Lemma 6.4 now hold with arbitrarily large $T>0$, we obtain (6.7) and (6.8), respectively.

Lemma 6.4. Let $d$ be defined in (6.18). There is $C_{0}>0$ such that for any $\varepsilon \in\left(0, \varepsilon_{*}\right], \kappa \in(0,1]$ and $T>0$, if $\boldsymbol{u}^{0} \in V$ is such that $U_{1}, U_{2}, F_{1}$ and $F_{2}$ defined in Theorem 6.2 are less than $\kappa^{2}$, and $\boldsymbol{u}(t)$ is a strong solution on $[0, T)$ satisfying $\boldsymbol{u}(0)=\boldsymbol{u}^{0}$ and

$$
\varepsilon|\boldsymbol{\nu}|_{\star}^{-3}\left\|A^{1 / 2} \boldsymbol{u}(t)\right\|_{L^{2}}^{2} \leq d \quad \text { for all } \quad t \in[0, T),
$$

then

$$
\varepsilon|\boldsymbol{\nu}|_{\star}^{-3}\left\|A^{1 / 2} \boldsymbol{u}(t)\right\|_{L^{2}}^{2} \leq C_{0} \kappa^{2} \quad \text { for all } \quad t \in[0, T) .
$$

Proof. For $t \in(0, T)$, taking the inner product of equation (4.19) with $A \boldsymbol{u}(t)$ and applying the estimate in Proposition 5.1 with $\alpha=1 / 4$, we have

$$
\begin{aligned}
& \frac{1}{2} \frac{d}{d t}\left\|A^{1 / 2} \boldsymbol{u}\right\|_{L^{2}}^{2}+\|A \boldsymbol{u}\|_{L^{2}}^{2} \leq|\langle B(\boldsymbol{u}, \boldsymbol{u}), A \boldsymbol{u}\rangle|+|\langle A \boldsymbol{u}, P \boldsymbol{f}\rangle| \\
& \leq \frac{1}{4}\|A \boldsymbol{u}\|_{L^{2}}^{2}+c_{3} \varepsilon^{1 / 2}|\boldsymbol{\nu}|_{\star}^{-3 / 2}\left\|A^{1 / 2} \boldsymbol{u}\right\|_{L^{2}}\|A \boldsymbol{u}\|_{L^{2}}^{2} \\
& \quad+C \varepsilon^{-1}\left(|\boldsymbol{\nu}|_{\star}^{-1}\|\boldsymbol{u}\|_{L^{2}}^{2}+\|\boldsymbol{u}\|_{L^{2}}\right)\left\|A^{1 / 2} \boldsymbol{u}\right\|_{L^{2}}^{2}+\frac{1}{4}\|A \boldsymbol{u}\|_{L^{2}}^{2}+\|P \boldsymbol{f}\|_{L^{2}}^{2},
\end{aligned}
$$

hence

$$
\begin{aligned}
& \frac{d}{d t}\left\|A^{1 / 2} \boldsymbol{u}\right\|_{L^{2}}^{2}+\left\{1-2 c_{3} \varepsilon^{1 / 2}|\boldsymbol{\nu}|_{\star}^{-3 / 2}\left\|A^{1 / 2} \boldsymbol{u}\right\|_{L^{2}}\right\}\|A \boldsymbol{u}\|_{L^{2}}^{2} \\
& \leq C \varepsilon^{-1}\left(|\boldsymbol{\nu}|_{\star}^{-1}\|\boldsymbol{u}\|_{L^{2}}^{2}+\|\boldsymbol{u}\|_{L^{2}}\right)\left\|A^{1 / 2} \boldsymbol{u}\right\|_{L^{2}}^{2}+2\|P \boldsymbol{f}\|_{L^{2}}^{2} .
\end{aligned}
$$

By assumption (6.26) and the $L^{2}$-estimate (6.21), it follows that

$$
\begin{aligned}
& \frac{d}{d t}\left\|A^{1 / 2} \boldsymbol{u}\right\|_{L^{2}}^{2}+\left\{1-2 c_{3} \sqrt{d}\right\}\|A \boldsymbol{u}\|_{L^{2}}^{2} \\
& \leq C_{3} \varepsilon^{-1}\left(\kappa^{2}+\kappa\right)|\boldsymbol{\nu}|_{\star}\left\|A^{1 / 2} \boldsymbol{u}\right\|_{L^{2}}^{2}+C \varepsilon^{\delta-2} \Lambda_{4},
\end{aligned}
$$

where $C_{3}$ and $C$ do not depend on $\kappa$. Since $2 c_{3} \sqrt{d}=1 / 2$ and $\kappa \leq 1$, we obtain

$$
\frac{d}{d t}\left\|A^{1 / 2} \boldsymbol{u}\right\|_{L^{2}}^{2}+\frac{1}{2}\|A \boldsymbol{u}\|_{L^{2}}^{2} \leq 2 C_{3} \varepsilon^{-1}|\boldsymbol{\nu}|_{\star}\left\|A^{1 / 2} \boldsymbol{u}\right\|_{L^{2}}^{2}+C \varepsilon^{\delta-2}|\boldsymbol{\nu}|_{\star}^{4} \Lambda_{4} .
$$

Consider the case $t<\min \left\{\varepsilon^{1-\delta} /|\boldsymbol{\nu}|_{\star}, T\right\}$. By (6.28) and (4.27),

$$
\frac{d}{d t}\left\|A^{1 / 2} \boldsymbol{u}\right\|_{L^{2}}^{2}+\frac{|\boldsymbol{\nu}|_{\star}}{2 c_{2}^{2} \varepsilon^{1-\delta}}\left\|A^{1 / 2} \boldsymbol{u}\right\|_{L^{2}}^{2} \leq C \varepsilon^{-1}|\boldsymbol{\nu}|_{\star}\left\|A^{1 / 2} \boldsymbol{u}\right\|_{L^{2}}^{2}+C \varepsilon^{\delta-2}|\boldsymbol{\nu}|_{\star}^{4} \Lambda_{4} .
$$


Applying Gronwall's inequality, one has

$$
\begin{aligned}
& \left\|A^{1 / 2} \boldsymbol{u}(t)\right\|_{L^{2}}^{2} \leq\left\|A^{1 / 2} \boldsymbol{u}^{0}\right\|_{L^{2}}^{2} e^{-c_{2}^{-2} \varepsilon^{\delta-1}|\boldsymbol{\nu}|_{\star} t / 2} \\
& \quad+\int_{0}^{t} e^{-c_{2}^{-2} \varepsilon^{\delta-1}|\boldsymbol{\nu}|_{\star}(t-\tau) / 2}\left(C \varepsilon^{-1}|\boldsymbol{\nu}|_{\star}\left\|A^{1 / 2} \boldsymbol{u}(\tau)\right\|_{L^{2}}^{2}+C \varepsilon^{\delta-2}|\boldsymbol{\nu}|_{\star}^{4} \Lambda_{4}\right) d \tau \\
& \leq\left\|A^{1 / 2} \boldsymbol{u}^{0}\right\|_{L^{2}}^{2} e^{-c_{2}^{-2} \varepsilon^{\delta-1}|\boldsymbol{\nu}|_{\star} t / 2}+C \varepsilon^{-1}|\boldsymbol{\nu}|_{\star} \int_{0}^{t}\left\|A^{1 / 2} \boldsymbol{u}(\tau)\right\|_{L^{2}}^{2} d \tau \\
& \quad+C \varepsilon^{\delta-2}|\boldsymbol{\nu}|_{\star}^{4} \Lambda_{4} \int_{0}^{t} e^{-c_{2}^{-2} \varepsilon^{\delta-1}|\boldsymbol{\nu}|_{\star}(t-\tau) / 2} d \tau
\end{aligned}
$$

To estimate $\int_{0}^{t}\left\|A^{1 / 2} \boldsymbol{u}(\tau)\right\|_{L^{2}}^{2} d \tau$ appearing above, we apply (6.22) with $t=0$ and $\theta=t$. It results in

$$
\left\|A^{1 / 2} \boldsymbol{u}(t)\right\|_{L^{2}}^{2} \leq C \varepsilon^{-1}|\boldsymbol{\nu}|_{\star}^{3}\left\{U_{1} e^{-c_{2}^{-2} \varepsilon^{\delta-1}|\boldsymbol{\nu}|_{\star} t / 2}+\left(\Lambda_{1}+\Lambda_{2}\right)+\Lambda_{4}\right\} .
$$

Therefore

$$
\left\|A^{1 / 2} \boldsymbol{u}(t)\right\|_{L^{2}}^{2} \leq C_{4} \varepsilon^{-1}|\boldsymbol{\nu}|_{\star}^{3}\left(\Lambda_{3}+\Lambda_{4}\right), \quad t<\min \left\{\varepsilon^{1-\delta} /|\boldsymbol{\nu}|_{\star}, T\right\}
$$

and, consequently,

$$
\left\|A^{1 / 2} \boldsymbol{u}(t)\right\|_{L^{2}}^{2} \leq 4 C_{4} \varepsilon^{-1}|\boldsymbol{\nu}|_{\star}^{3} \kappa^{2}, \quad t<\min \left\{\varepsilon^{1-\delta} /|\boldsymbol{\nu}|_{\star}, T\right\} .
$$

If $T>\varepsilon^{1-\delta} /|\boldsymbol{\nu}|_{\star}$, we now consider $t \in\left[\varepsilon^{1-\delta} /|\boldsymbol{\nu}|_{\star}, T\right)$. By (6.28),

$$
\frac{d}{d t}\left\|A^{1 / 2} \boldsymbol{u}\right\|_{L^{2}}^{2} \leq h \stackrel{\text { def }}{=} C\left(\varepsilon^{-1}|\boldsymbol{\nu}|_{\star}\left\|A^{1 / 2} \boldsymbol{u}\right\|_{L^{2}}^{2}+\varepsilon^{\delta-2}|\boldsymbol{\nu}|_{\star}^{4} \Lambda_{4}\right) .
$$

Then applying the uniform Gronwall inequality, the form quoted in Lemma 7.2 of [8], (for the proof, see $[6,24,26]$,) with $y=\left\|A^{1 / 2} \boldsymbol{u}(t)\right\|_{L^{2}}^{2}, g=0$ and $\tau=t-\varepsilon^{1-\delta} /|\boldsymbol{\nu}|_{\star}$, we have

$$
\left\|A^{1 / 2} \boldsymbol{u}(t)\right\|_{L^{2}}^{2} \leq\left\{\frac{1}{\varepsilon^{1-\delta} /|\boldsymbol{\nu}|_{\star}} \int_{t-\varepsilon^{1-\delta} /|\boldsymbol{\nu}|_{\star}}^{t}\left\|A^{1 / 2} \boldsymbol{u}(\tau)\right\|_{L^{2}}^{2} d \tau+\int_{t-\varepsilon^{1-\delta} /|\boldsymbol{\nu}|_{\star}}^{t} h(\tau) d \tau\right\} .
$$

It follows from (6.22) that

$$
\begin{aligned}
\int_{t-\varepsilon^{1-\delta} /|\boldsymbol{\nu}|_{\star}}^{t}\left\|A^{1 / 2} \boldsymbol{u}(\tau)\right\|_{L^{2}}^{2} d \tau & \leq C|\boldsymbol{\nu}|_{\star}^{2}\left(\Lambda_{1} e^{-c_{2}^{-2} \varepsilon^{\delta-1}|\boldsymbol{\nu}|_{\star}\left(t-\varepsilon^{1-\delta} /|\boldsymbol{\nu}|_{\star}\right)}+\Lambda_{2}\right) \\
& \leq C|\boldsymbol{\nu}|_{\star}^{2}\left(\Lambda_{1} e^{-c_{2}^{-2} \varepsilon^{\delta-1}|\boldsymbol{\nu}|_{\star} t}+\Lambda_{2}\right) .
\end{aligned}
$$

Consequently,

$$
\int_{t-\varepsilon^{1-\delta} /|\boldsymbol{\nu}|_{\star}}^{t} h(\tau) d \tau \leq C\left\{\varepsilon^{-1}|\boldsymbol{\nu}|_{\star}^{3}\left(\Lambda_{1} e^{-c_{2}^{-2} \varepsilon^{\delta-1}|\boldsymbol{\nu}|_{\star} t}+\Lambda_{2}\right)+\varepsilon^{\delta-2}|\boldsymbol{\nu}|_{\star}^{4} \Lambda_{4} \frac{\varepsilon^{1-\delta}}{|\boldsymbol{\nu}|_{\star}}\right\},
$$

thus

$$
\int_{t-\varepsilon^{1-\delta} /|\boldsymbol{\nu}|_{\star}}^{t} h(\tau) d \tau \leq C \varepsilon^{-1}|\boldsymbol{\nu}|_{\star}^{3}\left(\Lambda_{1} e^{-c_{2}^{-2} \varepsilon^{\delta-1}|\boldsymbol{\nu}|_{\star} t}+\Lambda_{4}\right) .
$$


Then (6.31) implies

$$
\begin{aligned}
\left\|A^{1 / 2} \boldsymbol{u}(t)\right\|_{L^{2}}^{2} \leq & C \varepsilon^{\delta-1}|\boldsymbol{\nu}|_{\star}^{3}\left(\Lambda_{1} e^{-c_{2}^{-2} \varepsilon^{\delta-1}|\boldsymbol{\nu}|_{\star} t}+\Lambda_{2}\right) \\
& +C \varepsilon^{-1}|\boldsymbol{\nu}|_{\star}^{3}\left(\Lambda_{1} e^{-c_{2}^{-2} \varepsilon^{\delta-1}|\boldsymbol{\nu}|_{\star} t}+\Lambda_{4}\right) .
\end{aligned}
$$

Hence

$$
\left\|A^{1 / 2} \boldsymbol{u}(t)\right\|_{L^{2}}^{2} \leq C_{5} \varepsilon^{-1}|\boldsymbol{\nu}|_{\star}^{3}\left(\Lambda_{1} e^{-c_{2}^{-2} \varepsilon^{\delta-1}|\boldsymbol{\nu}|_{\star} t}+\Lambda_{4}\right) \quad \text { for } t \in\left[\varepsilon^{1-\delta} /|\boldsymbol{\nu}|_{\star}, T\right),
$$

and, by (6.17),

$$
\left\|A^{1 / 2} \boldsymbol{u}(t)\right\|_{L^{2}}^{2} \leq 4 C_{5} \kappa^{2} \varepsilon^{-1}|\boldsymbol{\nu}|_{\star}^{3} \text { for } t \in\left[\varepsilon^{1-\delta} /|\boldsymbol{\nu}|_{\star}, T\right) .
$$

It follows from (6.30) and (6.33) that for all $t \in[0, T)$,

$$
\left\|A^{1 / 2} \boldsymbol{u}(t)\right\|_{L^{2}}^{2} \leq C_{0} \kappa^{2} \varepsilon^{-1}|\boldsymbol{\nu}|_{\star}^{3},
$$

where $C_{0}$ is independent of $\varepsilon, \kappa, \nu$. We obtain (6.27) and the proof is complete.

Remark 6.5. (a) If the friction coefficients on the top and bottom boundaries are larger than $C \varepsilon \nu$, i.e., when $\delta<1$, then $F_{0}$ can be much larger than $O(1)$ and $F_{1}$ can be much larger than $O\left(\varepsilon^{-1}\right)$; those two are typical conditions in the case $\delta=1$ (c.f. $[8,9,15]$ ). This effect was not quantified in $[4,12]$.

(b) By relations (4.8), (6.11) and inequality (6.14) we have

$$
\limsup _{t \rightarrow \infty}\|\boldsymbol{u}\|_{H^{1}}^{2} \leq C \varepsilon^{-1}|\boldsymbol{\nu}|_{\star}^{2} \Lambda_{4},
$$

which is independent of the initial data. This provides an estimate for the size of the global attractor (see $[9,15])$.

(c) As a consequence of (6.14), the norm $\left\|u_{+}-u_{-}\right\|_{L^{2}\left(\Gamma_{0}\right)}$ is of $O\left(\varepsilon^{-1}\right)$, that is, the relative velocity between two fluids on the common surface can be very large. One scenario for this in reality (ignoring the incompressibility requirements) is winds blowing over the water.

\section{Appendix A. Auxiliary Inequalities}

We recall some auxiliary inequalities for thin domains. Below, the domain $\Omega_{\varepsilon}$ will be either $\Omega_{+}^{\varepsilon}$ or $\Omega_{-}^{\varepsilon}$ introduced in section 2 , and, respectively, $h_{1}$ will be $h_{+}$or $h_{-}$, and the boundary $\Gamma_{1}$ will be either $\Gamma_{+}$or $\Gamma_{-}$. As usual, $C$ denotes a positive constant independent of $\varepsilon$.

Poincaré and trace inequalities-I. For any $\phi \in H^{1}\left(\Omega_{\varepsilon}\right)$, we have for $i=0,1$, that

$$
\begin{aligned}
& \|\phi\|_{L^{2}\left(\Omega_{\varepsilon}\right)} \leq C \varepsilon\left\|\partial_{3} \phi\right\|_{L^{2}\left(\Omega_{\varepsilon}\right)}+C \sqrt{\varepsilon}\|\phi\|_{L^{2}\left(\Gamma_{i}\right)}, \\
& \|\phi\|_{L^{2}\left(\Gamma_{i}\right)} \leq \frac{C}{\sqrt{\varepsilon}}\|\phi\|_{L^{2}\left(\Omega_{\varepsilon}\right)}+C \sqrt{\varepsilon}\left\|\partial_{3} \phi\right\|_{L^{2}\left(\Omega_{\varepsilon}\right)} .
\end{aligned}
$$

Poincaré and trace inequalities-II. If $\phi \in W^{1,2}\left(\Omega_{\varepsilon}\right)$ satisfies

$$
\int_{h_{0}\left(x^{\prime}\right)}^{h_{1}\left(x^{\prime}\right)} \phi\left(x^{\prime}, y_{3}\right) d y_{3}=0 \quad \text { for all } x^{\prime} \in \mathbb{T}^{2}
$$


then

$$
\begin{gathered}
\|\phi\|_{L^{2}\left(\Omega_{\varepsilon}\right)} \leq C \varepsilon\left\|\partial_{3} \phi\right\|_{L^{2}\left(\Omega_{\varepsilon}\right)}, \\
\|\phi\|_{L^{2}\left(\Gamma_{0} \cup \Gamma_{1}\right)} \leq C \sqrt{\varepsilon}\left\|\partial_{3} \phi\right\|_{L^{2}\left(\Omega_{\varepsilon}\right)} .
\end{gathered}
$$

For direct proofs of the above inequalities, see e.g. [9].

2D Ladyzhenskaya inequality. Let $\phi \in H^{1}\left(\Omega_{\varepsilon}\right)$ be independent of $x_{3}$, then

$$
\|\phi\|_{L^{4}\left(\Omega_{\varepsilon}\right)} \leq C \varepsilon^{-1 / 4}\|\phi\|_{L^{2}\left(\Omega_{\varepsilon}\right)}^{1 / 2}\|\phi\|_{H^{1}\left(\Omega_{\varepsilon}\right)}^{1 / 2} .
$$

2D Agmon inequality. Let $\phi \in H^{2}\left(\Omega_{\varepsilon}\right)$ be independent of $x_{3}$, then

$$
\|\phi\|_{L^{\infty}\left(\Omega_{\varepsilon}\right)} \leq C \varepsilon^{-1 / 2}\|\phi\|_{L^{2}\left(\Omega_{\varepsilon}\right)}^{1 / 2}\|\phi\|_{H^{2}\left(\Omega_{\varepsilon}\right)}^{1 / 2} .
$$

The following estimate for a product of $2 \mathrm{D}$ and $3 \mathrm{D}$ functions originates from [3]; here we cite the form in Lemma 6.3 of [8].

Cao-Titi inequality. Suppose $v, \phi \in H^{1}\left(\Omega_{\varepsilon}\right)$ and $v$ is independent of $x_{3}$. Then

$$
\|v \phi\|_{L^{2}\left(\Omega_{\varepsilon}\right)} \leq C \varepsilon^{-1 / 2}\|v\|_{L^{2}\left(\Omega_{\varepsilon}\right)}^{1 / 2}\|v\|_{H^{1}\left(\Omega_{\varepsilon}\right)}^{1 / 2}\|\phi\|_{L^{2}}^{1 / 2}\|\phi\|_{H^{1}\left(\Omega_{\varepsilon}\right)}^{1 / 2} .
$$

\section{REFERENCES}

[1] M. Bulíček, J. Málek, and K. R. Rajagopal. Navier's slip and evolutionary Navier-Stokes-like systems with pressure and shear-rate dependent viscosity. Indiana Univ. Math. J., 56(1):51-85, 2007.

[2] A. V. Busuioc and T. S. Ratiu. The second grade fluid and averaged Euler equations with Navier-slip boundary conditions. Nonlinearity, 16(3):1119-1149, 2003.

[3] C. Cao and E. S. Titi. Global well-posedness of the three-dimensional viscous primitive equations of large scale ocean and atmosphere dynamics. Ann. of Math. (2), 166(1):245-267, 2007.

[4] I. D. Chueshov, G. Raugel, and A. M. Rekalo. Interface boundary value problem for the Navier-Stokes equations in thin two-layer domains. J. Differential Equations, 208(2):449-493, 2005.

[5] P. Constantin and C. Foias. Navier-Stokes equations. Chicago Lectures in Mathematics. University of Chicago Press, Chicago, IL, 1988.

[6] C. Foiaş and G. Prodi. Sur le comportement global des solutions non-stationnaires des équations de NavierStokes en dimension 2. Rend. Sem. Mat. Univ. Padova, 39:1-34, 1967.

[7] L. T. Hoang. A basic inequality for the Stokes operator related to the Navier boundary condition. J. Differential Equations, 245(9):2585-2594, 2008.

[8] L. T. Hoang. Incompressible fluids in thin domains with Navier friction boundary conditions (I). J. Math. Fluid Mech., 12(3):435-472, 2010.

[9] L. T. Hoang and G. R. Sell. Navier-Stokes equations with Navier boundary conditions for an oceanic model. J. Dynam. Differential Equations, 22(3):563-616, 2010.

[10] J. Hron, C. Le Roux, J. Málek, and K. R. Rajagopal. Flows of incompressible fluids subject to Navier's slip on the boundary. Comput. Math. Appl., 56(8):2128-2143, 2008.

[11] C. Hu. Navier-Stokes equations in 3D thin domains with Navier friction boundary condition. J. Differential Equations, 236(1):133-163, 2007.

[12] C. Hu. Global strong solutions of Navier-Stokes equations with interface boundary in three-dimensional thin domains. Nonlinear Anal., 74(12):3964-3997, 2011.

[13] D. Iftimie. The 3D Navier-Stokes equations seen as a perturbation of the 2D Navier-Stokes equations. Bull. Soc. Math. France, 127(4):473-517, 1999. 
[14] D. Iftimie and G. Raugel. Some results on the Navier-Stokes equations in thin 3D domains. J. Differential Equations, 169(2):281-331, 2001. Special issue in celebration of Jack K. Hale's 70th birthday, Part 4 (Atlanta, GA/Lisbon, 1998).

[15] D. Iftimie, G. Raugel, and G. R. Sell. Navier-Stokes equations in thin 3D domains with Navier boundary conditions. Indiana Univ. Math. J., 56:1083-1156, 2007.

[16] D. Iftimie and F. Sueur. Viscous boundary layers for the Navier-Stokes equations with the Navier slip conditions. Arch. Ration. Mech. Anal., 199(1):145-175, 2011.

[17] I. Kukavica and M. Ziane. Regularity of the Navier-Stokes equation in a thin periodic domain with large data. Discrete Contin. Dyn. Syst., 16(1):67-86, 2006.

[18] I. Kukavica and M. Ziane. On the regularity of the Navier-Stokes equation in a thin periodic domain. $J$. Differential Equations, 234(2):485-506, 2007.

[19] J.-L. Lions, R. Temam, and S. Wang. Models for the coupled atmosphere and ocean. (CAO I,II). Comput. Mech. Adv., 1(1):120, 1993.

[20] J.-L. Lions, R. Temam, and S. H. Wang. Mathematical theory for the coupled atmosphere-ocean models. (CAO III). J. Math. Pures Appl. (9), 74(2):105-163, 1995.

[21] G. Raugel and G. R. Sell. Navier-Stokes equations in thin 3D domains. III. Existence of a global attractor. In Turbulence in fluid flows, volume 55 of IMA Vol. Math. Appl., pages 137-163. Springer, New York, 1993.

[22] G. Raugel and G. R. Sell. Navier-Stokes equations on thin 3D domains. I. Global attractors and global regularity of solutions. J. Amer. Math. Soc., 6(3):503-568, 1993.

[23] G. Raugel and G. R. Sell. Navier-Stokes equations on thin 3D domains. II. Global regularity of spatially periodic solutions. In Nonlinear partial differential equations and their applications. Collège de France Seminar, Vol. XI (Paris, 1989-1991), volume 299 of Pitman Res. Notes Math. Ser., pages 205-247. Longman Sci. Tech., Harlow, 1994.

[24] G. R. Sell and Y. You. Dynamics of evolutionary equations, volume 143 of Applied Mathematical Sciences. Springer-Verlag, New York, 2002.

[25] V. A. Solonnikov and V. E. Ščadilov. A certain boundary value problem for the stationary system of NavierStokes equations. Trudy Mat. Inst. Steklov., 125:196-210, 235, 1973. Boundary value problems of mathematical physics, 8 .

[26] R. Temam. Infinite-dimensional dynamical systems in mechanics and physics, volume 68 of Applied Mathematical Sciences. Springer-Verlag, New York, second edition, 1997.

[27] R. Temam. Navier-Stokes equations. AMS Chelsea Publishing, Providence, RI, 2001. Theory and numerical analysis, Reprint of the 1984 edition.

[28] R. Temam and M. Ziane. Navier-Stokes equations in three-dimensional thin domains with various boundary conditions. Adv. Differential Equations, 1(4):499-546, 1996.

[29] R. Temam and M. Ziane. Navier-Stokes equations in thin spherical domains. In Optimization methods in partial differential equations (South Hadley, MA, 1996), volume 209 of Contemp. Math., pages 281-314. Amer. Math. Soc., Providence, RI, 1997.

[30] R. Temam and M. Ziane. Some mathematical problems in geophysical fluid dynamics. In Handbook of mathematical fluid dynamics. Vol. III, pages 535-657. North-Holland, Amsterdam, 2004.

Department of Mathematics and Statistics, Texas Tech University, Box 41042 Lubbock, TX 79409-1042, U.S.A.

E-mail address: luan. hoang@ttu. edu 